\title{
The $B V$-energy of maps into a manifold: relaxation and density results
}

\author{
MARIANo Giaquinta AND DOMEnico MuCCI
}

\begin{abstract}
Let $\mathcal{Y}$ be a smooth compact oriented Riemannian manifold without boundary, and assume that its 1-homology group has no torsion. Weak limits of graphs of smooth maps $u_{k}: B^{n} \rightarrow \mathcal{Y}$ with equibounded total variation give rise to equivalence classes of Cartesian currents in cart $^{1,1}\left(B^{n} \times \mathcal{Y}\right)$ for which we introduce a natural $B V$-energy. Assume moreover that the first homotopy group of $\mathcal{Y}$ is commutative. In any dimension $n$ we prove that every element $T$ in cart $^{1,1}\left(B^{n} \times \mathcal{Y}\right)$ can be approximated weakly in the sense of currents by a sequence of graphs of smooth maps $u_{k}: B^{n} \rightarrow \mathcal{Y}$ with total variation converging to the $B V$-energy of $T$. As a consequence, we characterize the lower semicontinuous envelope of functions of bounded variations from $B^{n}$ into $\mathcal{Y}$.
\end{abstract}

Mathematics Subject Classification (2000): 49Q15 (primary); 49Q20 (secondary).

In this paper we deal with sequences of smooth maps $u_{k}: B^{n} \rightarrow \mathcal{Y}$ with equibounded total variation

$$
\sup _{k} \mathcal{E}_{1,1}\left(u_{k}\right)<\infty, \quad \mathcal{E}_{1,1}\left(u_{k}\right):=\int_{B^{n}}\left|D u_{k}\right| d x
$$

and their limit points. Here $B^{n}$ is the unit ball in $\mathbb{R}^{n}$ and $\mathcal{Y}$ is a smooth oriented Riemannian manifold of dimension $M \geq 1$, isometrically embedded in $\mathbb{R}^{N}$ for some $N \geq 2$. We shall assume that $\mathcal{Y}$ is compact, connected, without boundary. In addition, we assume that the integral 1-homology group $H_{1}(\mathcal{Y}):=H_{1}(\mathcal{Y} ; \mathbb{Z})$ has no torsion.

Modulo passing to a subsequence the $(n, 1)$-currents $G_{u_{k}}$, integration over the graphs of $u_{k}$ of $n$-forms with at most one vertical differential, converge to a current $T \in \operatorname{cart}^{1,1}\left(B^{n} \times \mathcal{Y}\right)$, see Section 2 below. To every $T \in \operatorname{cart}^{1,1}\left(B^{n} \times \mathcal{Y}\right)$ it corresponds a function $u_{T} \in B V\left(B^{n}, \mathcal{Y}\right)$, i.e., $u_{T} \in B V\left(B^{n}, \mathbb{R}^{N}\right)$ such that $u_{T}(x) \in \mathcal{Y}$ for $\mathcal{L}^{n}$-a.e. $x \in B^{n}$, compare [14, Vol. I, Section 4.2] [14, Vol. II, Section 5.4]. Also, the weak convergence $G_{u_{k}} \rightarrow T$ yields the convergence $u_{k} \rightarrow$ $u_{T}$ weakly in the $B V$-sense.

Received June 12, 2006; accepted in revised form October 17, 2006. 
In order to analyze the weak limit currents, it is relevant first to consider the case $n=1$. Therefore in Section 1 we study some of the structure properties of 1-dimensional Cartesian currents in $B^{1} \times \mathcal{Y}$, i.e., of currents in $\operatorname{cart}\left(B^{1} \times \mathbb{R}^{N}\right)$ with support spt $T \subset \bar{B}^{1} \times \mathcal{Y}$, compare [14, Vol. I]. In the simple case $\mathcal{Y}=S^{1}$, the unit circle in $\mathbb{R}^{2}$, and in any dimension $n$, for any current $T \in \operatorname{cart}\left(B^{n} \times S^{1}\right)$ we can find a sequence of smooth maps $\left\{u_{k}\right\} \subset C^{1}\left(B^{n}, S^{1}\right)$ such that $G_{u_{k}}$ weakly converges to $T$ and the area of the graph of the $u_{k}$ 's converges to the mass of $T$, i.e., $\mathbf{M}\left(G_{u_{k}}\right) \rightarrow \mathbf{M}(T)$, see [13] and [14, Vol. II, Section 6.2.2]. However, in case of general target manifolds, and even in dimension $n=1$, a gap phenomenon occurs. More precisely, setting

$\tilde{\mathbf{M}}(T):=\inf \left\{\liminf _{k \rightarrow \infty} \mathbf{M}\left(G_{u_{k}}\right) \mid\left\{u_{k}\right\} \subset C^{1}\left(B^{1}, \mathcal{Y}\right), G_{u_{k}} \rightarrow T\right.$ weakly in $\left.\mathcal{D}_{1}\left(B^{1} \times \mathcal{Y}\right)\right\}$,

there exist currents $T \in \operatorname{cart}\left(B^{1} \times \mathcal{Y}\right)$ for which

$$
\mathbf{M}(T)<\tilde{\mathbf{M}}(T),
$$

i.e., for every smooth sequence $\left\{u_{k}\right\} \subset C^{1}\left(B^{1}, \mathcal{Y}\right)$ such that $G_{u_{k}} \rightarrow T$ weakly in $\mathcal{D}_{1}\left(B^{1} \times \mathcal{Y}\right)$ we have that

$$
\liminf _{k \rightarrow \infty} \mathbf{M}\left(G_{u_{k}}\right) \geq \mathbf{M}(T)+C
$$

where $C>0$ is an absolute constant and, we recall, the mass of $G_{u_{k}}$ is the area of the graph of $u_{k}$

$$
\mathbf{M}\left(G_{u_{k}}\right)=\mathcal{A}\left(u_{k}\right):=\int_{B^{1}} \sqrt{1+\left|D u_{k}\right|^{2}} d x
$$

In order to deal with this gap phenomenon, we introduce the class $\operatorname{cart}^{1,1}\left(B^{1} \times \mathcal{Y}\right)$ of equivalence classes of currents in $\operatorname{cart}\left(B^{1} \times \mathcal{Y}\right)$, where the equivalence relation is given by

$$
T \sim \widetilde{T} \Longleftrightarrow T(\omega)=\widetilde{T}(\omega) \quad \forall \omega \in \mathcal{Z}^{1,1}\left(B^{1} \times \mathcal{Y}\right)
$$

see Definition 1.6. Here $\mathcal{Z}^{1,1}\left(B^{1} \times \mathcal{Y}\right)$ denotes the class of smooth forms $\omega \in$ $\mathcal{D}^{1}\left(B^{1} \times \mathcal{Y}\right)$ such that $d_{y} \omega^{(1)}=0$, where $d=d_{x}+d_{y}$ denotes the splitting into a horizontal and a vertical differential, and $\omega^{(1)}$ is the component of $\omega$ with exactly one vertical differential. In other words $\operatorname{cart}^{1,1}\left(B^{1} \times \mathcal{Y}\right)$ is the class of vertical homological representatives of the elements of $\operatorname{cart}\left(B^{1} \times \mathcal{Y}\right)$. Notice that if $\mathcal{Y}=S^{1}$, actually cart $^{1,1}\left(B^{1} \times S^{1}\right)$ agrees with the class $\operatorname{cart}\left(B^{1} \times S^{1}\right)$. We then introduce on $\operatorname{cart}^{1,1}\left(B^{1} \times \mathcal{Y}\right)$ the following energy

$$
\mathcal{A}(T):=\int_{B^{1}} \sqrt{1+\left|\nabla u_{T}(x)\right|^{2}} d x+\left|D^{C} u_{T}\right|\left(B^{1}\right)+\sum_{x \in J_{c}(T)} \mathcal{L}_{T}(x),
$$


where $\nabla u_{T}$ and $D^{C} u_{T}$ are respectively the absolutely continuous and the Cantor part of the distributional derivative of the underlying function $u_{T} \in B V\left(B^{1}, \mathcal{Y}\right)$, and the countable set $J_{c}(T)$ is the union

$$
J_{c}(T):=J_{u_{T}} \cup\left\{x_{i}: i=1, \ldots, I\right\}
$$

of the discontinuity set $J_{u_{T}}$ of $u_{T}$ and of the finite set of points $x_{i}$ where the mass of $T$ concentrates.

In the above formula, $\mathcal{L}_{T}(x)$ denotes the minimal length $\mathcal{L}(\gamma)$ among all Lipschitz curves $\gamma:[0,1] \rightarrow \mathcal{Y}$, with end points equal to the one-sided approximate limits of $u_{T}$ on $x \in J_{c}(T)$, such that their image current $\gamma_{\#} \llbracket(0,1) \rrbracket$ is equal to the 1-dimensional restriction $\widehat{\pi}_{\#}(T\llcorner\{x\} \times \mathcal{Y})$ of $T$ over the point $x$. In the case $\mathcal{Y}=S^{1}$, it turns out that $\mathcal{A}(T)$ agrees with the mass of $T$, compare [13] and [14, Vol. II, Section 6.2.2].

We will show that the functional $T \mapsto \mathcal{A}(T)$ is lower semicontinuous in $\operatorname{cart}^{1,1}\left(B^{1} \times \mathcal{Y}\right)$, Theorem 1.7 , and that for every $T$ there exists a sequence of smooth maps $\left\{u_{k}\right\} \subset C^{1}\left(B^{1}, \mathcal{Y}\right)$ such that $G_{u_{k}} \rightarrow T$ and $\mathbf{M}\left(G_{u_{k}}\right) \rightarrow \mathcal{A}(T)$ as $k \rightarrow \infty$, Theorem 1.8. As a consequence, we conclude that $\mathcal{A}(T)$ coincides with the relaxed area functional

$$
\widetilde{\mathcal{A}}(T):=\inf \left\{\liminf _{k \rightarrow \infty} \mathcal{A}\left(u_{k}\right) \mid\left\{u_{k}\right\} \subset C^{1}\left(B^{1}, \mathcal{Y}\right), \quad G_{u_{k}} \rightarrow T\right\} .
$$

In Section 2, we deal with the $n$-dimensional case, $n \geq 2$, introducing the class $\operatorname{cart}^{1,1}\left(B^{n} \times \mathcal{Y}\right)$ of vertical homological representatives. The $B V$-energy of a current $T \in \operatorname{cart}^{1,1}\left(B^{n} \times \mathcal{Y}\right)$ is then defined by

$$
\mathcal{E}_{1,1}(T):=\int_{B^{n}}\left|\nabla u_{T}(x)\right| d x+\left|D^{C} u_{T}\right|\left(B^{n}\right)+\int_{J_{c}(T)} \mathcal{L}_{T}(x) d \mathcal{H}^{n-1}(x),
$$

see Definition 2.10, where $J_{c}(T)$ is the countably $\mathcal{H}^{n-1}$-rectifiable subset of $B^{n}$ given by the union of the Jump set $J_{u_{T}}$ of $u_{T}$ and of the $(n-1)$-rectifiable set of mass-concentration of $T$. Finally, the integrand $\mathcal{L}_{T}(x)$ is defined as above, by taking into account that the 1 -dimensional restriction $\widehat{\pi}_{\#}(T\llcorner\{x\} \times \mathcal{Y})$ of $T$ is well-defined for $\mathcal{H}^{n-1}$-a.e. point $x \in J_{C}(T)$.

Notice that, if $T=G_{u}$, where $u: B^{n} \rightarrow \mathcal{Y}$ is smooth or at least in $W^{1,1}$, then $\mathcal{E}_{1,1}\left(G_{u}\right)=\mathcal{E}_{1,1}(u)$. Moreover, in the case $\mathcal{Y}=S^{1}$, we have $\operatorname{cart}^{1,1}\left(B^{n} \times S^{1}\right)=$ $\operatorname{cart}\left(B^{n} \times S^{1}\right)$ and, due to the absence of gap phenomenon, the functional $\mathcal{E}_{1,1}(T)$ agrees with the parametric variational integral associated to the total variation integral, see Definition 2.5, and can be dealt with as in [13], see also [14, Vol. II, Section 6.2], [8], [19]. The functional $T \mapsto \mathcal{E}_{1,1}(T)$ turns out to be lower semicontinuous in $\operatorname{cart}^{1,1}\left(B^{n} \times \mathcal{Y}\right)$, see Theorem 2.12 and Section 3. Moreover, assuming in addition that the first homotopy group $\pi_{1}(\mathcal{Y})$ is commutative, in Section 4 and Section 5 we will prove in any dimension $n \geq 2$ that for every $T \in \operatorname{cart}^{1,1}\left(B^{n} \times \mathcal{Y}\right)$ there exists a sequence of smooth maps $\left\{u_{k}\right\} \subset C^{1}\left(B^{n}, \mathcal{Y}\right)$ such that $G_{u_{k}} \rightarrow T$ 
and $\mathcal{E}_{1,1}\left(u_{k}\right) \rightarrow \mathcal{E}_{1,1}(T)$ as $k \rightarrow \infty$, Theorem 2.13. Consequently, we show that a closure-compactness property holds in $\operatorname{cart}^{1,1}\left(B^{n} \times \mathcal{Y}\right)$, Theorem 2.17 . We stress that the commutativity hypothesis on $\pi_{1}(\mathcal{Y})$ cannot be removed, see Remark 5.2.

In Section 6, extending the classical notion of total variation of vector-valued maps, compare e.g. [1], we introduce in a natural way the total variation of functions $u \in B V\left(B^{n}, \mathcal{Y}\right)$, given by

$$
\mathcal{E}_{T V}(u):=\int_{B^{n}}|\nabla u(x)| d x+\left|D^{C} u\right|\left(B^{n}\right)+\int_{J_{u}} \mathcal{H}^{1}\left(l_{x}\right) d \mathcal{H}^{n-1}(x),
$$

where, for any $x \in J_{u}$, we let $\mathcal{H}^{1}\left(l_{x}\right)$ denote the length of a geodesic arc $l_{x}$ in $\mathcal{Y}$ with initial and final points $u^{-}(x)$ and $u^{+}(x)$. Extending the density result of Bethuel [5], in Theorem 6.5 we will show that for every $u \in B V\left(B^{n}, \mathcal{Y}\right)$ we can find a sequence of maps $\left\{u_{k}\right\} \subset R_{1}^{\infty}\left(B^{n}, \mathcal{Y}\right)$ such that $u_{k} \rightarrow u$ as $k \rightarrow \infty$ weakly in the $B V$-sense and

$$
\lim _{k \rightarrow \infty} \int_{B^{n}}\left|D u_{k}\right| d x=\mathcal{E}_{T V}(u) .
$$

If $n=1$, the class $R_{1}^{\infty}\left(B^{n}, \mathcal{Y}\right)$ agrees with $C^{1}\left(B^{n}, \mathcal{Y}\right)$. If $n \geq 2$, it is given by all the maps $u \in W^{1,1}\left(B^{n}, \mathcal{Y}\right)$ which are smooth except on a singular set which is discrete, if $n=2$, and is the finite union of smooth $(n-2)$-dimensional subsets of $B^{n}$ with smooth boundary, if $n \geq 3$. Therefore, if $\pi_{1}(\mathcal{Y})=0$, we obtain that smooth maps in $C^{1}\left(B^{n}, \mathcal{Y}\right)$ are dense in $B V\left(B^{n}, \mathcal{Y}\right)$ in the strong sense above mentioned.

However, in Section 7 we will show that $\mathcal{E}_{T V}(u)$ does not agree with the relaxed of the total variation

$\widetilde{\mathcal{E}_{T V}}(u):=\inf \left\{\liminf _{k \rightarrow \infty} \int_{B^{n}}\left|D u_{k}\right| d x \mid\left\{u_{k}\right\} \subset C^{1}\left(B^{n}, \mathcal{Y}\right), u_{k} \rightarrow u\right.$ weakly in the $B V$-sense $\}$ if $n \geq 2$, and we have $\widetilde{\mathcal{E}_{T V}}(u)<\infty$, Theorem 7.3, and that

$$
\widetilde{\mathcal{E}_{T V}}(u)=\inf \left\{\mathcal{E}_{1,1}(T) \mid T \in \mathcal{T}_{u}\right\}
$$

Theorem 7.4, where $\mathcal{T}_{u}$ is the class of Cartesian currents $T$ in $\operatorname{cart}^{1,1}\left(B^{n} \times \mathcal{Y}\right)$ with underlying $B V$-function $u_{T}$ equal to $u$, this way obtaining the representation formula

$\widetilde{\mathcal{E}_{T V}}(u)=\int_{B^{n}}|\nabla u(x)| d x+\left|D^{C} u\right|\left(B^{n}\right)+\inf \left\{\int_{J_{C}(T)} \mathcal{L}_{T}(x) d \mathcal{H}^{n-1}(x) \mid T \in \mathcal{T}_{u}\right\}$.

We finally specify the above relaxation results to $u \in W^{1,1}\left(B^{n}, \mathcal{Y}\right)$ and/or $\mathcal{Y}=S^{1}$, recovering in particular previous results in [13, 8], and [19].

Acknowledgement. The second author thanks the Research Center Ennio De Giorgi of the Scuola Normale Superiore of Pisa for the hospitality during the preparation of this paper. 


\section{Cartesian currents in dimension one}

In this section we discuss some features of 1-dimensional Cartesian currents in $B^{1} \times \mathcal{Y}$ and, in particular, we discuss a gap phenomenon and the relaxed area functional.

First let us introduce a few notation about $B V$-functions and Cartesian currents in the general context $B^{n} \times \mathcal{Y}$.

Vector valued $\boldsymbol{B} \boldsymbol{V}$-functions. Let $u: B^{n} \rightarrow \mathbb{R}^{N}$ be a function in $B V\left(B^{n}, \mathbb{R}^{N}\right)$, i.e., $u=\left(u^{1}, \ldots u^{N}\right)$ with all components $u^{j} \in B V\left(B^{n}\right)$. The Jump set of $u$ is the countably $\mathcal{H}^{n-1}$-rectifiable set $J_{u}$ in $B^{n}$ given by the union of the complements of the Lebesgue sets of the $u^{j}$ 's. Let $v=v_{u}(x)$ be a unit vector in $\mathbb{R}^{n}$ orthogonal to $J_{u}$ at $\mathcal{H}^{n-1}$-a.e. point $x \in J_{u}$. Let $u^{ \pm}(x)$ denote the one-sided approximate limits of $u$ on $J_{u}$, so that for $\mathcal{H}^{n-1}$-a.e. point $x \in J_{u}$

$$
\lim _{\rho \rightarrow 0^{+}} \rho^{-n} \int_{B_{\rho}^{ \pm}(x)}\left|u(x)-u^{ \pm}(x)\right| d x=0,
$$

where $B_{\rho}^{ \pm}(x):=\left\{y \in B_{\rho}(x): \pm\langle y-x, v(x)\rangle \geq 0\right\}$. Note that a change of sign of $v$ induces a permutation of $u^{+}$and $u^{-}$and that only for scalar functions there is a canonical choice of the sign of $v$ which ensures that $u^{+}(x)>u^{-}(x)$. The distributional derivative of $u$ is the sum of a "gradient" measure, which is absolutely continuous with respect to the Lebesgue measure, of a "jump" measure, concentrated on a set that is $\sigma$-finite with respect to the $\mathcal{H}^{n-1}$-measure, and of a "Cantor-type" measure. More precisely,

$$
D u=D^{a} u+D^{J} u+D^{C} u,
$$

where

$$
D^{a} u=\nabla u \cdot d x, \quad D^{J} u=\left(u^{+}(x)-u^{-}(x)\right) \otimes v(x) \mathcal{H}^{n-1}\left\llcorner J_{u},\right.
$$

$\nabla u:=\left(\nabla_{1} u, \ldots, \nabla_{n} u\right)$ being the approximate gradient of $u$, compare e.g. [2] or $\left[14\right.$, Vol. I]. We also recall that $\left\{u_{k}\right\}$ is said to converge to $u$ weakly in the $B V$ sense, $u_{k} \rightarrow u$, if $u_{k} \rightarrow u$ strongly in $L^{1}\left(B^{n}, \mathbb{R}^{N}\right)$ and $D u_{k} \rightarrow D u$ weakly in the sense of (vector-valued) measures. We will finally denote

$$
B V\left(B^{n}, \mathcal{Y}\right):=\left\{u \in B V\left(B^{n}, \mathbb{R}^{N}\right) \mid u(x) \in \mathcal{Y} \quad \text { for } \mathcal{L}^{n} \text {-a.e. } x \in B^{n}\right\} .
$$

Cartesian currents. The class of Cartesian currents $\operatorname{cart}\left(B^{n} \times \mathbb{R}^{N}\right)$, compare [14, Vol. I], is defined as the class of integer multiplicity rectifiable currents $T$ in $\mathcal{R}_{n}\left(B^{n} \times \mathbb{R}^{N}\right)$ which have no inner boundary, $\partial T\left\llcorner B^{n} \times \mathbb{R}^{N}=0\right.$, have finite mass, $\mathbf{M}(T)<\infty$, and are such that

$$
\|T\|_{1}<\infty, \quad \pi_{\#}(T)=\llbracket\left[B^{n} \rrbracket \quad \text { and } \quad T^{\overline{0} 0} \geq 0,\right.
$$


where

$$
\|T\|_{1}:=\sup \left\{T(\varphi(x, y)|y| d x) \mid \varphi \in C_{c}^{0}\left(B^{n} \times \mathbb{R}^{N}\right) \text { and }\|\varphi\| \leq 1\right\}
$$

and $T^{\overline{0} 0}$ is the Radon measure in $B^{n} \times \mathbb{R}^{N}$ given by

$$
T^{\overline{0} 0}(\varphi(x, y))=T(\varphi(x, y) d x) \quad \forall \varphi \in C_{c}^{0}\left(B^{n} \times \mathbb{R}^{N}\right) .
$$

Finally, here and in the sequel $\pi: \mathbb{R}^{n+N} \rightarrow \mathbb{R}^{n}$ and $\widehat{\pi}: \mathbb{R}^{n+N} \rightarrow \mathbb{R}^{N}$ denote the projections onto the first $n$ and the last $N$ coordinates, respectively.

It is shown in [14, Vol. I] that for every $T \in \operatorname{cart}\left(B^{n} \times \mathbb{R}^{N}\right)$ there exists a function $u_{T} \in B V\left(B^{n}, \mathbb{R}^{N}\right)$ such that

$$
T(\phi(x, y) d x)=\int_{B^{n}} \phi\left(x, u_{T}(x)\right) d x
$$

for all $\phi \in C^{0}\left(B^{n} \times \mathbb{R}^{N}\right)$ such that $|\phi(x, y)| \leq C(1+|y|)$, and

$$
(-1)^{n-i} T\left(\varphi(x) \widehat{d x^{i}} \wedge d y^{j}\right)=\left\langle D_{i} u_{T}^{j}, \varphi\right\rangle:=-\int_{B^{n}} u_{T}^{j}(x) \cdot D_{i} \varphi(x) d x
$$

for all $\varphi \in C_{c}^{1}\left(B^{n}\right)$, where

$$
\widehat{d x^{i}}:=d x^{1} \wedge \cdots d x^{i-1} \wedge d x^{i-1} \wedge \cdots \wedge d x^{n} .
$$

In particular, we have $\|T\|_{1}=\left\|u_{T}\right\|_{L^{1}\left(B^{n}, \mathbb{R}^{N}\right)}$.

Definition 1.1. If $n=1$ we set

$$
\operatorname{cart}\left(B^{1} \times \mathcal{Y}\right):=\left\{T \in \operatorname{cart}\left(B^{1} \times \mathbb{R}^{N}\right) \mid \operatorname{spt} T \subset \bar{B}^{1} \times \mathcal{Y}\right\} .
$$

Notice that the class $\operatorname{cart}\left(B^{1} \times \mathcal{Y}\right)$ contains the weak limits of sequences of graphs of smooth maps $u_{k}: B^{1} \rightarrow \mathcal{Y}$ with equibounded $W^{1,1}$-energies. Moreover, it is closed under weak convergence in $\mathcal{D}_{1}\left(B^{1} \times \mathcal{Y}\right)$ with equibounded masses. Finally, the $B V$-function $u_{T}$ associated to currents $T$ in $\operatorname{cart}\left(B^{1} \times \mathcal{Y}\right)$ clearly belongs to $B V\left(B^{1}, \mathcal{Y}\right)$.

Restriction over one point. Let $T \in \operatorname{cart}\left(B^{1} \times \mathcal{Y}\right)$. Since $T$ has finite mass, $\eta \mapsto T\left(\chi_{B_{r}(x)} \wedge \eta\right)$, where $x \in B^{1}$ and $0<r<1-|x|$, defines a current in $\mathcal{D}_{1}(\mathcal{Y})$. The 1 -dimensional restriction of $T$ over the point $x$

$$
\widehat{\pi}_{\#}\left(T\llcorner\{x\} \times \mathcal{Y}) \in \mathcal{D}_{1}(\mathcal{Y})\right.
$$

is the limit

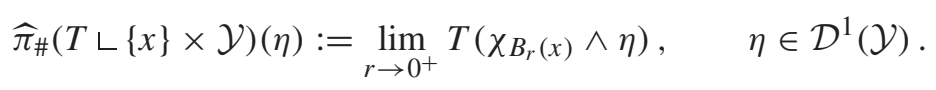


Canonical decomposition. There is a canonical way to decompose a current $T \in$ $\operatorname{cart}\left(B^{1} \times \mathcal{Y}\right)$. We first observe that the 1-dimensional restriction of $T$ over any point $x$ in the jump set $J_{u_{T}}$ of $u_{T}$ is given by

$$
\widehat{\pi}_{\#}\left(T\llcorner\{x\} \times \mathcal{Y})=\Gamma_{x},\right.
$$

$\Gamma_{x}$ being a 1-dimensional integral chain on $\mathcal{Y}$ such that $\partial \Gamma_{x}=\delta_{u_{T}^{+}(x)}-\delta_{u_{T}^{-}(x)}$, where $u_{T}^{+}(x)$ and $u_{T}^{-}(x)$ here and in the sequel denote the right and left limits of $u_{T}$ at $x$, respectively. Therefore, by applying Federer's decomposition theorem [9], we find an indecomposable 1-dimensional integral chain $\gamma_{x}$ on $\mathcal{Y}$, satisfying $\partial \gamma_{x}=\delta_{u_{T}^{+}(x)}-\delta_{u_{T}^{-}(x)}$, and an integral 1-cycle $C_{x}$ in $\mathcal{Y}$, satisfying $\partial C_{x}=0$, such that

$$
\Gamma_{x}=\gamma_{x}+C_{x} \quad \text { and } \quad \mathbf{M}\left(\Gamma_{x}\right)=\mathbf{M}\left(\gamma_{x}\right)+\mathbf{M}\left(C_{x}\right) .
$$

Currents associated to graphs of $\boldsymbol{B} \boldsymbol{V}$-functions. Next we associate to any $T \in$ $\operatorname{cart}\left(B^{1} \times \mathcal{Y}\right)$ a current $G_{T} \in \mathcal{D}_{1}\left(B^{1} \times \mathcal{Y}\right)$ carried by the graph of the function $u_{T} \in B V\left(B^{1}, \mathcal{Y}\right)$ corresponding to $T$, and acting in a linear way on forms $\omega$ in $\mathcal{D}^{1}\left(B^{1} \times \mathcal{Y}\right)$ as follows. We first split $\omega=\omega^{(0)}+\omega^{(1)}$ according to the number of vertical differentials, so that

$$
\omega^{(0)}=\phi(x, y) d x \quad \text { and } \quad \omega^{(1)}=\sum_{j=1}^{N} \phi^{j}(x, y) d y^{j}
$$

for some $\phi, \phi^{j} \in C_{0}^{\infty}\left(B^{1} \times \mathcal{Y}\right)$. We then decompose $G_{T}$ into its absolutely continuous, Cantor, and Jump parts

$$
G_{T}:=T^{a}+T^{C}+T^{J}
$$

and define $T^{C}\left(\omega^{(0)}\right)=T^{J}\left(\omega^{(0)}\right)=0$ and

$$
\begin{aligned}
T^{a}\left(\omega^{(0)}\right) & :=\int_{B^{1}} \phi\left(x, u_{T}(x)\right) d x \\
T^{a}\left(\omega^{(1)}\right) & :=\sum_{j=1}^{N} \int_{B^{1}} \phi^{j}\left(x, u_{T}(x)\right) \nabla u_{T}^{j}(x) d x \\
T^{C}\left(\omega^{(1)}\right) & :=\sum_{j=1}^{N}\left\langle D^{C} u_{T}^{j}, \phi^{j}\left(\cdot, u_{T}(\cdot)\right)\right\rangle \\
T^{J}\left(\omega^{(1)}\right) & :=\sum_{j=1}^{N} \int_{J_{u_{T}}}\left(\int_{\gamma_{x}} \phi^{j}(x, y) d y^{j}\right) \cdot v(x) d \mathcal{H}^{0}(x) .
\end{aligned}
$$

Here, $\gamma_{x}$ is the indecomposable 1-dimensional integral chain defined by means of the 1-dimensional restriction of $T$ over the point $x \in J_{u_{T}}$, see (1.2). 
Notice that the definition of $G_{T}$ obviously depends on $\gamma_{x}$ and hence, in conclusion, on the current $T \in \operatorname{cart}\left(B^{1} \times \mathcal{Y}\right)$. Moreover, we readily infer that the mass of $G_{T}$ is given by

$$
\mathbf{M}\left(G_{T}\right)=\mathbf{M}\left(T^{a}\right)+\mathbf{M}\left(T^{C}\right)+\mathbf{M}\left(T^{J}\right)
$$

where

$$
\begin{aligned}
& \mathbf{M}\left(T^{a}\right)=\int_{B^{1}} \sqrt{1+\left|\nabla u_{T}(x)\right|^{2}} d x, \\
& \mathbf{M}\left(T^{C}\right)=\left|D^{C} u_{T}\right|\left(B^{1}\right), \\
& \mathbf{M}\left(T^{J}\right)=\int_{J_{u_{T}}} \mathcal{H}^{1}\left(\gamma_{x}\right) d \mathcal{H}^{0}(x) .
\end{aligned}
$$

A density result. We recall from [14] that if $u: B^{1} \rightarrow \mathcal{Y}$ is smooth, or at least e.g. $u \in W^{1,1}\left(B^{1}, \mathcal{Y}\right)$, the current $G_{u}$ integration of 1 -forms in $\mathcal{D}^{1}\left(B^{1} \times \mathcal{Y}\right)$ over the rectifiable graph of $u$ is defined in a weak sense by $G_{u}:=(I d \bowtie u)_{\#} \llbracket B^{1} \rrbracket$, i.e., by letting $G_{u}(\omega)=(I d \bowtie u)^{\#}(\omega)$ for every $\omega \in \mathcal{D}^{1}\left(B^{1} \times \mathcal{Y}\right)$, where $(I d \bowtie$ $u)(x):=(x, u(x))$. Moreover, the mass of $G_{u}$ agrees with the area $\mathcal{A}(u)$ of the graph of $u$

$$
\mathbf{M}\left(G_{u}\right)=\mathcal{A}(u):=\int_{B^{1}} \sqrt{1+|D u(x)|^{2}} d x .
$$

By a straightforward adaptation of the proof of Theorem 1.8 below, we readily obtain the following strong density result for the mass of $G_{T}$.

Proposition 1.2. For every $T \in \operatorname{cart}\left(B^{1} \times \mathcal{Y}\right)$ there exists a sequence of smooth maps $\left\{u_{k}\right\} \subset C^{1}\left(B^{1}, \mathcal{Y}\right)$ such that $u_{k} \rightarrow u_{T}$ weakly in the $B V$-sense, $G_{u_{k}} \rightarrow G_{T}$ weakly in $\mathcal{D}_{1}\left(B^{1} \times \mathcal{Y}\right)$ and $\mathbf{M}\left(G_{u_{k}}\right) \rightarrow \mathbf{M}\left(G_{T}\right)$ as $k \rightarrow \infty$.

Vertical Homology. Let now $\mathcal{Z}^{1,1}\left(B^{1} \times \mathcal{Y}\right)$ denote the class of vertically closed forms

$$
\mathcal{Z}^{1,1}\left(B^{1} \times \mathcal{Y}\right):=\left\{\omega \in \mathcal{D}^{1}\left(B^{1} \times \mathcal{Y}\right) \mid d_{y} \omega^{(1)}=0\right\}
$$

where $d=d_{x}+d_{y}$ denotes the splitting of the exterior differential $d$ into a horizontal and a vertical differential. We say that $T_{k} \rightarrow T$ weakly in $\mathcal{Z}_{1,1}\left(B^{1} \times \mathcal{Y}\right)$ if $T_{k}(\omega) \rightarrow T(\omega)$ for every $\omega \in \mathcal{Z}^{1,1}\left(B^{1} \times \mathcal{Y}\right)$.

Homological vertical part. By Proposition 1.2, since by Stokes' theorem $\partial G_{u_{k}}\left\llcorner B^{1} \times\right.$ $\mathcal{Y}=0$, whereas $G_{u_{k}} \rightarrow G_{T}$, we obtain that

$$
\partial G_{T}\left\llcorner B^{1} \times \mathcal{Y}=0 .\right.
$$

Remark 1.3. In higher dimension $n \geq 2$ in general $G_{T}$ has a non-zero boundary, i.e., $\partial G_{T}\left\llcorner B^{n} \times \mathcal{Y} \neq 0\right.$, see Remark 2.2. 
Setting then

$$
S_{T}:=T-G_{T},
$$

by (1.1) we infer that $S_{T}(\phi(x, y) d x)=0$ and $S_{T}(d \phi)=0$ for every $\phi \in$ $C_{0}^{\infty}\left(B^{1} \times \mathcal{Y}\right)$. Therefore, by homological reasons, since

$$
\inf \left\{\mathbf{M}(C) \mid C \in \mathcal{Z}_{1}(\mathcal{Y}), C \text { is non trivial in } \mathcal{Y}\right\}>0,
$$

similarly to [14, Vol. II, Section 5.3.1] we infer that

$$
S_{T}=\sum_{i=1}^{I} \delta_{x_{i}} \times C_{i} \quad \text { on } \quad \mathcal{Z}^{1,1}\left(B^{1} \times \mathcal{Y}\right),
$$

where $\left\{x_{i}: i=1, \ldots, I\right\}$ is a finite disjoint set of points in $B^{1}$, possibly intersecting the Jump set $J_{u_{T}}$, and $C_{i}$ is a non-trivial homological integral 1-cycle in $\mathcal{Y}$. Notice that the integral 1-homology group $H_{1}(\mathcal{Y})$ is finitely generated.

Remark 1.4. Setting

$$
S_{T, \text { sing }}:=T-G_{T}-\sum_{i=1}^{I} \delta_{x_{i}} \times C_{i},
$$

it turns out that $S_{T, \text { sing }}$ is nonzero only possibly on forms $\omega$ with non-zero vertical component, $\omega^{(1)} \neq 0$, and such that $d_{y} \omega^{(1)} \neq 0$. Therefore, $S_{T \text {, sing }}$ is a homologically trivial integer multiplicity rectifiable current in $\mathcal{R}_{1}\left(B^{1} \times \mathcal{Y}\right)$.

Consequently, setting for $T \in \operatorname{cart}\left(B^{1} \times \mathcal{Y}\right)$

$$
T^{H}:=\sum_{i=1}^{I} \delta_{x_{i}} \times C_{i},
$$

$T$ decomposes into the absolutely continuous, Cantor, Jump, Homological, and Singular parts,

$$
T=T^{a}+T^{C}+T^{J}+T^{H}+S_{T, \text { sing }} .
$$

Gap phenomenon. However, a gap phenomenon occurs in $\operatorname{cart}\left(B^{1} \times \mathcal{Y}\right)$. More precisely, if we set

$\tilde{\mathbf{M}}(T):=\inf \left\{\liminf _{k \rightarrow \infty} \mathbf{M}\left(G_{u_{k}}\right) \mid\left\{u_{k}\right\} \subset C^{1}\left(B^{1}, \mathcal{Y}\right), G_{u_{k}} \rightarrow T\right.$ weakly in $\left.\mathcal{D}_{1}\left(B^{1} \times \mathcal{Y}\right)\right\}$,

we see that there exist Cartesian currents $T \in \operatorname{cart}\left(B^{1} \times \mathcal{Y}\right)$ for which

$$
\mathbf{M}(T)<\tilde{\mathbf{M}}(T) .
$$

For example, as in [14, Vol. I, Section 4.2.5], if $T=G_{u}+\delta_{0} \times C$, where $u \equiv P \in \mathcal{Y}$ is a constant map and $C \in \mathcal{Z}^{1}(\mathcal{Y})$ is a 1-cycle in $\mathcal{Y}$, it readily follows that for every 
smooth sequence $\left\{u_{k}\right\} \subset C^{1}\left(B^{1}, \mathcal{Y}\right)$ such that $G_{u_{k}} \rightarrow T$ weakly in $\mathcal{D}_{1}\left(B^{1} \times \mathcal{Y}\right)$ we have that

$$
\liminf _{k \rightarrow \infty} \mathbf{M}\left(G_{u_{k}}\right) \geq \mathbf{M}(T)+2 d, \quad d:=\operatorname{dist}_{\mathcal{Y}}(P, \operatorname{spt} C),
$$

where dist $\mathcal{Y}$ denotes the geodesic distance in $\mathcal{Y}$.

Remark 1.5. This gap phenomenon is due to the structure of the area integrand $u \mapsto \sqrt{1+|D u|^{2}}$, and it is typical of integrands with linear growth of the gradient, e.g., the total variation integrand $u \mapsto|D u|$, since the images of smooth approximating sequences may have to "connect" the point $P$ to the cycle $C$, this way paying a cost in term of the distance $d$. This does not happen e.g. for the Dirichlet integrand $u \mapsto \frac{1}{2}|D u|^{2}$ in dimension 2, compare [15]. In this case, in fact, the connection from one point $P$ to any 2-cycle $C \in \mathcal{Z}_{2}(\mathcal{Y})$ can be obtained by means of "cylinders" of small 2-dimensional mapping area and, therefore, of small Dirichlet integral, on account of Morrey's $\varepsilon$-conformality theorem.

Homological theory. In order to study the currents which arise as weak limits of graphs of smooth maps $u_{k}: B^{1} \rightarrow \mathcal{Y}$ with equibounded total variations, $\sup _{k}\left\|D u_{k}\right\|_{L^{1}}<\infty$, the previous facts lead us to consider vertical homology equivalence classes of currents in $\operatorname{cart}\left(B^{1} \times \mathcal{Y}\right)$. More precisely, we give the following

Definition 1.6. We denote by $\operatorname{cart}^{1,1}\left(B^{1} \times \mathcal{Y}\right)$ the set of equivalence classes of currents in $\operatorname{cart}\left(B^{1} \times \mathcal{Y}\right)$, where

$$
T \sim \widetilde{T} \Longleftrightarrow T(\omega)=\widetilde{T}(\omega) \quad \forall \omega \in \mathcal{Z}^{1,1}\left(B^{1} \times \mathcal{Y}\right)
$$

If $T \sim \widetilde{T}$, then the underlying $B V$-functions coincide, i.e., $u_{T}=u_{\widetilde{T}}$. Therefore, we have $T^{a}=\widetilde{T}^{a}$ and $T^{C}=\widetilde{T}^{C}$, whereas in general $T^{J} \neq \widetilde{T}^{J}$. However, we have that

$$
T^{J}+T^{H}=\widetilde{T}^{J}+\widetilde{T}^{H} \quad \text { on } \quad \mathcal{Z}^{1,1}\left(B^{1} \times \mathcal{Y}\right) .
$$

Jump-concentration points. For future use, we let

$$
J_{c}(T):=J_{u_{T}} \cup\left\{x_{i}: i=1, \ldots, I\right\}
$$

denote the set of points of jump and concentration, where the $x_{i}$ 's are given by (1.3). We infer that $J_{c}(T)$ is an at most countable set which does not depend on the representative $T$, i.e., $J_{c}(T)=J_{c}(\widetilde{T})$ if $T \sim \widetilde{T}$. By extending the notion of 1-dimensional restriction $\widehat{\pi}_{\#}(T\llcorner\{x\} \times \mathcal{Y})$ to equivalence classes, we infer that $\widehat{\pi}_{\#}\left(T\llcorner\{x\} \times \mathcal{Y})=0\right.$ if $x \notin J_{c}(T)$. As to jump-concentration points, letting

$$
\mathcal{Z}^{1}(\mathcal{Y}):=\left\{\eta \in \mathcal{D}^{1}(\mathcal{Y}) \mid d_{y} \eta=0\right\}
$$

if $x \in J_{u_{T}}$, with $x \neq x_{i}$, we infer that

$$
\widehat{\pi}_{\#}\left(T\llcorner\{x\} \times \mathcal{Y})=\gamma_{x} \quad \text { on } \quad \mathcal{Z}^{1}(\mathcal{Y}),\right.
$$


where $\gamma_{x}$ is the indecomposable 1-dimensional integral chain defined by (1.2), and if $x=x_{i}$, see (1.4),

$$
\widehat{\pi}_{\#}\left(T\llcorner\{x\} \times \mathcal{Y})=\gamma_{x_{i}}+C_{i} \quad \text { on } \quad \mathcal{Z}^{1}(\mathcal{Y}),\right.
$$

where $C_{i} \in \mathcal{Z}_{1}(\mathcal{Y})$ is the non-trivial 1-cycle defined by (1.3), and $\gamma_{x_{i}}=0$ if $x_{i} \notin J_{u_{T}}$.

Vertical minimal connection. For every Cartesian current $T \in \operatorname{cart}^{1,1}\left(B^{1} \times \mathcal{Y}\right)$ and every point $x \in J_{c}(T)$ we will denote by

$$
\begin{aligned}
\Gamma_{T}(x):=\{\gamma \in \operatorname{Lip}([0,1], \mathcal{Y}) \mid & \gamma(0)=u_{T}^{-}(x), \gamma(1)=u_{T}^{+}(x), \\
& \gamma_{\#} \llbracket(0,1) \rrbracket(\eta)=\widehat{\pi}_{\#}\left(T\llcorner\{x\} \times \mathcal{Y})(\eta) \forall \eta \in \mathcal{Z}^{1}(\mathcal{Y})\right\}
\end{aligned}
$$

the family of all smooth curves $\gamma$ in $\mathcal{Y}$, with end points $u_{T}^{ \pm}(x)$, such that their image current $\gamma_{\#} \llbracket(0,1) \rrbracket$ agrees with the 1-dimensional restriction $\widehat{\pi}_{\#}(T\llcorner\{x\} \times$ $\mathcal{Y})$ on closed 1 -forms in $\mathcal{Z}^{1}(\mathcal{Y})$. Moreover, we denote by

$$
\mathcal{L}_{T}(x):=\inf \left\{\mathcal{L}(\gamma) \mid \gamma \in \Gamma_{T}(x)\right\}, \quad x \in J_{c}(T),
$$

the minimal length of curves $\gamma$ connecting the "vertical part" of $T$ over $x$ to the graph of $u_{T}$. For future use, we remark that the infimum in (1.6) is attained, i.e.,

$$
\forall x \in J_{c}(T), \quad \exists \gamma \in \Gamma_{T}(x) \quad: \quad \mathcal{L}(\gamma)=\mathcal{L}_{T}(x)
$$

Relaxed area functional. We finally introduce the functional

$$
\mathcal{A}(T, B):=\int_{B} \sqrt{1+\left|\nabla u_{T}(x)\right|^{2}} d x+\left|D^{C} u_{T}\right|(B)+\int_{J_{C}(T) \cap B} \mathcal{L}_{T}(x) d \mathcal{H}^{0}(x)
$$

for every Borel set $B \subset B^{1}$, and we let

$$
\mathcal{A}(T):=\mathcal{A}\left(T, B^{1}\right) .
$$

Notice that for every $T \in \operatorname{cart}^{1,1}\left(B^{1} \times \mathcal{Y}\right)$ we have

$$
\min \{\mathbf{M}(\widetilde{T}): \widetilde{T} \sim T\} \leq \mathcal{A}(T) .
$$

Main results. We first prove the following lower semicontinuity property.

Theorem 1.7. Let $T \in \operatorname{cart}^{1,1}\left(B^{1} \times \mathcal{Y}\right)$. For every sequence of smooth maps $\left\{u_{k}\right\} \subset C^{1}\left(B^{1}, \mathcal{Y}\right)$ such that $G_{u_{k}} \rightarrow T$ weakly in $\mathcal{Z}_{1,1}\left(B^{1} \times \mathcal{Y}\right)$, we have

$$
\liminf _{k \rightarrow \infty} \mathbf{M}\left(G_{u_{k}}\right) \geq \mathcal{A}(T) .
$$

Then we prove the following density result. 
Theorem 1.8. Let $T \in \operatorname{cart}^{1,1}\left(B^{1} \times \mathcal{Y}\right)$. There exists a sequence of smooth maps $\left\{u_{k}\right\} \subset C^{1}\left(B^{1}, \mathcal{Y}\right)$ such that $G_{u_{k}} \rightarrow T$ weakly in $\mathcal{Z}_{1,1}\left(B^{1} \times \mathcal{Y}\right)$ and $\mathbf{M}\left(G_{u_{k}}\right) \rightarrow$ $\mathcal{A}(T)$ as $k \rightarrow \infty$.

As a consequence, if we denote, in the same spirit as Lebesgue's relaxed area, $\widetilde{\mathcal{A}}(T):=\inf \left\{\liminf _{k \rightarrow \infty} \mathcal{A}\left(u_{k}\right) \mid\left\{u_{k}\right\} \subset C^{1}\left(B^{1}, \mathcal{Y}\right), G_{u_{k}} \rightarrow T\right.$ weakly in $\left.\mathcal{Z}_{1,1}\left(B^{1} \times \mathcal{Y}\right)\right\}$, by Theorems 1.7 and 1.8 we readily conclude that

$$
\mathcal{A}(T)=\tilde{\mathcal{A}}(T) \quad \forall T \in \operatorname{cart}^{1,1}\left(B^{1} \times \mathcal{Y}\right) .
$$

Properties. From Theorems 1.7 and 1.8, (1.8) and the closure of the class $\operatorname{cart}\left(B^{1} \times\right.$ $\mathcal{Y})$ we infer:

(i) the functional $T \mapsto \mathcal{A}(T)$ is lower semicontinuous in $\operatorname{cart}^{1,1}\left(B^{1} \times \mathcal{Y}\right)$ with respect to the weak convergence in $\mathcal{Z}_{1,1}\left(B^{1} \times \mathcal{Y}\right)$;

(ii) the class cart $^{1,1}\left(B^{1} \times \mathcal{Y}\right)$ is closed and compact under weak convergence in $\mathcal{Z}_{1,1}\left(B^{1} \times \mathcal{Y}\right)$ with equibounded $\mathcal{A}$-energies.

We finally notice that similar properties hold if one considers the total variation integrand $u \mapsto|D u|$ instead of the area integrand $u \mapsto \sqrt{1+|D u|^{2}}$. In particular, setting

$$
\mathcal{E}_{1,1}(T):=\int_{B^{1}}\left|\nabla u_{T}(x)\right| d x+\left|D^{C} u_{T}\right|\left(B^{1}\right)+\int_{J_{c}(T)} \mathcal{L}_{T}(x) d \mathcal{H}^{0}(x),
$$

for every $T \in \operatorname{cart}^{1,1}\left(B^{1} \times \mathcal{Y}\right)$ we have

$$
\begin{gathered}
\mathcal{E}_{1,1}(T)=\inf \left\{\liminf _{k \rightarrow \infty} \int_{B^{1}}\left|D u_{k}\right| d x \mid\left\{u_{k}\right\} \subset C^{1}\left(B^{1}, \mathcal{Y}\right),\right. \\
\left.G_{u_{k}} \rightarrow T \quad \text { weakly in } \mathcal{Z}_{1,1}\left(B^{1} \times \mathcal{Y}\right)\right\} .
\end{gathered}
$$

Remark 1.9. For future use, we denote

$$
\mathcal{Y}_{\varepsilon}:=\left\{y \in \mathbb{R}^{N} \mid \operatorname{dist}(y, \mathcal{Y}) \leq \varepsilon\right\}
$$

the $\varepsilon$-neighborhood of $\mathcal{Y}$ and we observe that, since $\mathcal{Y}$ is smooth, there exists $\varepsilon_{0}>0$ such that for $0<\varepsilon \leq \varepsilon_{0}$ the nearest point projection $\Pi_{\varepsilon}$ of $\mathcal{Y}_{\varepsilon}$ onto $\mathcal{Y}$ is a well defined Lipschitz map with Lipschitz constant $L_{\varepsilon} \rightarrow 1^{+}$as $\varepsilon \rightarrow 0^{+}$. Note that for $0<\varepsilon \leq \varepsilon_{0}$ the set $\mathcal{Y}_{\varepsilon}$ is equivalent to $\mathcal{Y}$ in the sense of the algebraic topology. In particular, we have

$$
\pi_{1}\left(\mathcal{Y}_{\varepsilon}\right)=\pi_{1}(\mathcal{Y})
$$


Proof of Theorem 1.7. Let $\left\{x_{i}\right\}_{i>I} \subset B^{1}$ be the at most countable set of discontinuity points in $J_{u_{T}} \backslash\left\{x_{i}: i=1, \ldots, I\right\}$, see (1.4). By the properties of $\mathcal{Y}$ we have

$$
\mathcal{L}_{T}\left(x_{i}\right) \leq C \cdot\left|u_{T}^{+}\left(x_{i}\right)-u_{T}^{-}\left(x_{i}\right)\right| \quad \forall i>I,
$$

where $C=C(\mathcal{Y})>0$ is an absolute constant, see (1.6). Therefore, since

$$
\left|D^{J} u_{T}\right|\left(B^{1}\right)=\sum_{i=1}^{\infty}\left|u_{T}^{+}\left(x_{i}\right)-u_{T}^{-}\left(x_{i}\right)\right|<\infty,
$$

for every $\varepsilon>0$ we find $l(\varepsilon)>I$ such that

$$
\sum_{i=l(\varepsilon)+1}^{\infty} \mathcal{L}_{T}\left(x_{i}\right)<\varepsilon
$$

After rearranging in an increasing way the set $\left\{x_{i}: i \leq l(\varepsilon)\right\}$, and setting $x_{0}=-1$, $x_{l(\varepsilon)+1}=1$, we let

$$
2 \delta=2 \delta(\varepsilon):=\min \left\{\left|x_{i}-x_{i+1}\right|: i=0, \ldots, l(\varepsilon)\right\}>0 .
$$

For $i \in\{1, \ldots, l(\varepsilon)\}$, due to the weak convergence $u_{k} \rightarrow u_{T}$ in the $B V$-sense, possibly passing to a subsequence, we find the existence of sequences of points $\left.a_{k}^{i} \in\right] x_{i}-\delta / k, x_{i}\left[\right.$ and $\left.b_{k}^{i} \in\right] x_{i}, x_{i}+\delta / k[$ such that

$$
\operatorname{dist} \mathcal{Y}\left(u_{k}\left(a_{k}^{i}\right), u_{T}^{-}\left(x_{i}\right)\right)<\frac{1}{k} \quad \text { and } \quad \operatorname{dist} \mathcal{Y}\left(u_{k}\left(b_{k}^{i}\right), u_{T}^{+}\left(x_{i}\right)\right)<\frac{1}{k}
$$

for every $k$, where dist $\mathcal{Y}$ denotes the geodesic distance in $\mathcal{Y}$.

Let $\gamma_{k}^{i}:[0,1] \rightarrow \mathcal{Y}$ be the Lipschitz reparametrization with constant velocity of the smooth curve $u_{k \mid\left[a_{k}^{i}, b_{k}^{i}\right]}$. From the weak convergence $G_{u_{k}} \rightarrow T$ we infer that

$$
\gamma_{k \#}^{i} \llbracket(0,1) \rrbracket(\eta) \rightarrow \widehat{\pi}_{\#}\left(T\llcorner\{x\} \times \mathcal{Y})(\eta) \quad \forall \eta \in \mathcal{Z}^{1}(\mathcal{Y})\right.
$$

as $k \rightarrow \infty$, where $\widehat{\pi}_{\#}(T\llcorner\{x\} \times \mathcal{Y})$ is the previously defined restriction of $T$ over $x$. Moreover, by connecting the end points $u_{k}\left(a_{k}^{i}\right)$ and $u_{k}\left(b_{k}^{i}\right)$ with $u_{T}^{-}\left(x_{i}\right)$ and $u_{T}^{+}\left(x_{i}\right)$, respectively, due to (1.10) we find a sequence of Lipschitz arcs $\tilde{\gamma}_{k}^{i}$ : $[0,1] \rightarrow \mathcal{Y}$, with end points $\tilde{\gamma}_{k}^{i}(0)=u_{T}^{-}\left(x_{i}\right)$ and $\tilde{\gamma}_{k}^{i}(1)=u_{T}^{+}\left(x_{i}\right)$, such that $\left(\tilde{\gamma}_{k \#}^{i} \llbracket(0,1) \rrbracket-\gamma_{k \#}^{i} \llbracket(0,1) \rrbracket\right)(\eta) \rightarrow 0$ for every $\eta \in \mathcal{Z}^{1}(\mathcal{Y})$ as $k \rightarrow \infty$ and

$$
\mathcal{L}\left(\widetilde{\gamma}_{k}^{i}\right) \leq \mathcal{L}\left(\gamma_{k}^{i}\right)+\frac{2}{k} \quad \forall k
$$

By the construction we also infer that $\left\{\widetilde{\gamma}_{k}^{i}\right\}_{k}$ is a sequence of equibounded and equicontinuous maps. Therefore, by Ascoli's theorem, possibly passing to a subsequence, we find that $\tilde{\gamma}_{k}^{i}$ converges uniformly to a Lipschitz arc $\tilde{\gamma}^{i}:[0,1] \rightarrow \mathcal{Y}$, with end points $u_{T}^{\mp}\left(x_{i}\right)$, satisfying by (1.11)

$$
\widetilde{\gamma}_{\#}^{i} \llbracket(0,1) \rrbracket(\eta)=\widehat{\pi}_{\#}\left(T\llcorner\{x\} \times \mathcal{Y})(\eta) \quad \forall \eta \in \mathcal{Z}^{1}(\mathcal{Y}) .\right.
$$


We then obtain that $\tilde{\gamma}^{i} \in \Gamma_{T}\left(x_{i}\right)$, according to the definition (1.5). Moreover, by the lower semicontinuity of the length functional with respect to the uniform convergence, we have

$$
\mathcal{L}\left(\tilde{\gamma}^{i}\right) \leq \liminf _{k \rightarrow \infty} \mathcal{L}\left(\widetilde{\gamma}_{k}^{i}\right)
$$

By (1.6) and by the above estimates we conclude that

$$
\mathcal{L}_{T}\left(x_{i}\right) \leq \liminf _{k \rightarrow \infty} \mathcal{L}\left(\gamma_{k}^{i}\right) \quad \forall i=1, \ldots, l(\varepsilon) .
$$

Now, since by the weak $B V$-convergence of $u_{k} \rightarrow u_{T}$ we have

$$
\int_{B^{1}} \sqrt{1+\left|\nabla u_{T}(x)\right|^{2}} d x+\left|D^{C} u_{T}\right|\left(B^{1}\right) \leq \liminf _{k \rightarrow \infty} \mathcal{A}\left(u_{k}\right),
$$

by the previous argument, taking into account (1.9) and (1.12), we readily infer that

$$
\mathcal{A}(T)-\varepsilon \leq \liminf _{k \rightarrow \infty} \mathcal{A}\left(u_{k}\right)
$$

and hence the assertion, by letting $\varepsilon \searrow 0$.

Proof of Theorem 1.8. Let $\left\{x_{i}\right\}_{i>I}, l(\varepsilon)$ and $\delta=\delta(\varepsilon)$ be defined as in the proof of Theorem 1.7, so that (1.9) holds true. Let $\gamma^{i} \in \Gamma_{T}\left(x_{i}\right)$ be such that $\mathcal{L}\left(\gamma^{i}\right) \leq \mathcal{L}_{T}\left(x_{i}\right)+\varepsilon \cdot 2^{-i}$, see (1.5) and (1.6). For fixed $\delta \in(0, \delta(\varepsilon))$, and for every $i=1, \ldots, l(\varepsilon)$, we first define $u_{\delta}^{\varepsilon}:\left[x_{i}-\delta, x_{i}+\delta\right] \rightarrow \mathcal{Y}$ by reparametrising with the same orientation the arc $\gamma_{i}$, i.e.,

$$
u_{\delta}^{\varepsilon}(x):=\gamma^{i}\left(\frac{1}{2}+\frac{1}{2 \delta}\left(x-x_{i}\right)\right) .
$$

Setting $\left.I_{i}:=\right] x_{i}+\delta, x_{i+1}-\delta\left[\right.$ if $i=1, \ldots, l(\varepsilon)-1$, and $\left.I_{1}:=\right]-1, x_{1}-\delta\left[, I_{l(\varepsilon)}:=\right.$ ]$x_{l(\varepsilon)}+\delta, 1\left[\right.$, we then extend $u_{\delta}^{\varepsilon}$ to the whole of $B^{1}$ by letting $u_{\delta}^{\varepsilon}(x):=u_{T}\left(\Psi_{i}(x)\right)$ if $x \in I_{i}$ for some $i=0, \ldots, l(\varepsilon)$, where $\Psi_{i}$ is the bijective and increasing affine map between the intervals $I_{i}$ and $] x_{i}, x_{i+1}[$. We then apply a mollification procedure to the function $u_{\delta}^{\varepsilon}$, defining this way a smooth map $v_{\delta}^{\varepsilon}: B^{1} \rightarrow \mathbb{R}^{N}$ such that

$$
\left\|v_{\delta}^{\varepsilon}-u_{\delta}^{\varepsilon}\right\|_{L^{1}\left(B^{1}\right)} \leq \delta \quad \text { and } \quad \int_{B^{1}}\left|D v_{\delta}^{\varepsilon}\right| d x \leq\left|D u_{\delta}^{\varepsilon}\right|\left(B^{1}\right)+\delta .
$$

Since $u_{T}$ is continuous outside the Jump set $J_{u_{T}}$ and (1.9) holds true, for every $\sigma>0$ we find $\eta=\eta(\sigma, \delta, \varepsilon)>0$ such that, in the a.e. sense,

$$
\forall x, y \in B^{1}, \quad|x-y|<\eta \Longrightarrow\left|u_{\delta}^{\varepsilon}(x)-u_{\delta}^{\varepsilon}(y)\right|<\sigma+\varepsilon .
$$

As a consequence, we may and do define $v_{\delta}^{\varepsilon}$ in such a way that in particular

$$
\operatorname{dist}\left(v_{\delta}^{\varepsilon}(x), \mathcal{Y}\right)<\varepsilon \quad \forall x \in B^{1} .
$$

Setting now $w_{\delta}^{\varepsilon}:=\Pi_{\varepsilon} \circ v_{\delta}^{\varepsilon}: B^{1} \rightarrow \mathcal{Y}$, compare Remark 1.9, taking first $\delta$ small with respect to $\varepsilon$, and letting then $\varepsilon \rightarrow 0$, by a diagonal procedure we find a smooth approximating sequence. 


\section{Cartesian currents, $B V$-energy and weak limits}

In this section we deal with the weak limits of graphs of smooth maps $u_{k}: B^{n} \rightarrow \mathcal{Y}$ with equibounded $W^{1,1}$-energies. We first state a few preliminary results.

Homological facts. Since $H_{1}(\mathcal{Y})$ has no torsion, there are generators $\left[\gamma_{1}\right], \ldots,\left[\gamma_{\bar{s}}\right]$, i.e. integral 1-cycles in $\mathcal{Z}_{1}(\mathcal{Y})$, such that

$$
H_{1}(\mathcal{Y})=\left\{\sum_{s=1}^{\bar{s}} n_{s}\left[\gamma_{s}\right] \mid n_{s} \in \mathbb{Z}\right\},
$$

see e.g. [14], Vol. I, Section 5.4.1. By de Rham's theorem the first real homology group is in duality with the first cohomology group $H_{d R}^{1}(\mathcal{Y})$, the duality being given by the natural pairing

$$
\langle[\gamma],[\omega]\rangle:=\gamma(\omega)=\int_{\gamma} \omega, \quad[\gamma] \in H_{1}(\mathcal{Y} ; \mathbb{R}), \quad[\omega] \in H_{d R}^{1}(\mathcal{Y}) .
$$

We will then denote by $\left[\omega^{1}\right], \ldots,\left[\omega^{\bar{s}}\right]$ a dual basis in $H_{d R}^{1}(\mathcal{Y})$ so that $\gamma_{s}\left(\omega^{r}\right)=$ $\delta_{s r}$, where $\delta_{s r}$ denotes the Kronecker symbols.

$\mathcal{D}_{\boldsymbol{n}, \mathbf{1}}$-currents. For $p=1, \ldots, n$, every differential $p$-form $\omega \in \mathcal{D}^{p}\left(B^{n} \times \mathcal{Y}\right)$ splits as a sum $\omega=\sum_{j=0}^{\bar{p}} \omega^{(j)}$, where $\bar{p}:=\min (p, M), M=\operatorname{dim}(\mathcal{Y})$, and the $\omega^{(j)}$ 's are the $p$-forms that contain exactly $j$ differentials in the vertical $\mathcal{Y}$ variables. We denote by $\mathcal{D}^{p, 1}\left(B^{n} \times \mathcal{Y}\right)$ the subspace of $\mathcal{D}^{p}\left(B^{n} \times \mathcal{Y}\right)$ of $p$ forms of the type $\omega=\omega^{(0)}+\omega^{(1)}$, and by $\mathcal{D}_{p, 1}\left(B^{n} \times \mathcal{Y}\right)$ the dual space of $\mathcal{D}^{p, 1}\left(B^{n} \times \mathcal{Y}\right)$. Every $(p, 1)$-current $T \in \mathcal{D}_{p, 1}\left(B^{n} \times \mathcal{Y}\right)$ splits as $T=T_{(0)}+T_{(1)}$, where $T_{(j)}(\omega):=T\left(\omega^{(j)}\right)$. For example, if $u \in W^{1,1}\left(B^{n}, \mathcal{Y}\right)$, then $G_{u}$ is an $(n, 1)$-current in $\mathcal{D}_{n, 1}\left(B^{n} \times \mathcal{Y}\right)$ defined in an approximate sense by

$$
G_{u}:=(I d \bowtie u)_{\#} \llbracket B^{n} \rrbracket,
$$

where $(I d \bowtie u)(x):=(x, u(x))$, compare [14], see also [4].

Weak $\mathcal{D}_{\boldsymbol{n}, \mathbf{1}}$-convergence. If $\left\{T_{k}\right\} \subset \mathcal{D}_{n, 1}\left(B^{n} \times \mathcal{Y}\right)$, we say that $\left\{T_{k}\right\}$ converges weakly in $\mathcal{D}_{n, 1}\left(B^{n} \times \mathcal{Y}\right), T_{k} \rightarrow T$, if $T_{k}(\omega) \rightarrow T(\omega)$ for every $\omega \in \mathcal{D}^{n, 1}\left(B^{n} \times \mathcal{Y}\right)$. Trivially, the class $\mathcal{D}_{n, 1}\left(B^{n} \times \mathcal{Y}\right)$ is closed under weak convergence.

$\mathcal{E}_{\mathbf{1}, \mathbf{1}}$-norm. For $\omega \in \mathcal{D}^{n, 1}\left(B^{n} \times \mathcal{Y}\right)$ and $T \in \mathcal{D}_{n, 1}\left(B^{n} \times \mathcal{Y}\right)$ we set

$$
\begin{aligned}
& \|\omega\|_{\mathcal{E}_{1,1}}:=\max \left\{\sup _{x, y} \frac{\left|\omega^{(0)}(x, y)\right|}{1+|y|}, \int_{B^{n}} \sup _{y}\left|\omega^{(1)}(x, y)\right| d x\right\}, \\
& \|T\|_{\mathcal{E}_{1,1}}:=\sup \left\{T(\omega) \mid \omega \in \mathcal{D}^{n, 1}\left(B^{n} \times \mathcal{Y}\right),\|\omega\|_{\mathcal{E}_{1,1}} \leq 1\right\} .
\end{aligned}
$$


It is not difficult to show that $\|T\|_{\mathcal{E}_{1,1}}$ is a norm on $\left\{T \in \mathcal{D}_{n, 1}\left(B^{n} \times \mathcal{Y}\right):\|T\|_{\mathcal{E}_{1,1}}<\right.$ $\infty$. Moreover, $\|\cdot\|_{\mathcal{E}_{1,1}}$ is weakly lower semicontinuous in $\mathcal{D}_{n, 1}$, so that $\{T \in$ $\left.\mathcal{D}_{n, 1}\left(B^{n} \times \mathcal{Y}\right):\|T\|_{\mathcal{E}_{1,1}}<\infty\right\}$ is closed under weak $\mathcal{D}_{n, 1}$-convergence with equibounded $\mathcal{E}_{1,1}$-norms. Finally, if $\sup _{k}\left\|T_{k}\right\|_{\mathcal{E}_{1,1}}<\infty$ there is a subsequence that weakly converges to some $T \in \mathcal{D}_{n, 1}\left(B^{n} \times \mathcal{Y}\right)$ with $\|T\|_{\mathcal{E}_{1,1}}<\infty$.

Boundaries. The exterior differential $d$ splits into a horizontal and a vertical differential $d=d_{x}+d_{y}$. Of course $\partial_{x} T(\omega):=T\left(d_{x} \omega\right)$ defines a boundary operator $\partial_{x}: \mathcal{D}_{n, 1}\left(B^{n} \times \mathcal{Y}\right) \rightarrow \mathcal{D}_{n-1,1}\left(B^{n} \times \mathcal{Y}\right)$. Now, for any $\omega \in \mathcal{D}^{n-1,1}\left(B^{n} \times \mathcal{Y}\right), d_{y} \omega$ belongs to $\mathcal{D}^{n, 1}\left(B^{n} \times \mathcal{Y}\right)$ if and only if $d_{y} \omega^{(1)}=0$. Then $\partial_{y} T$ makes sense only as an element of the dual space of $\mathcal{Z}^{n-1,1}\left(B^{n} \times \mathcal{Y}\right)$, where

$$
\mathcal{Z}^{p, 1}\left(B^{n} \times \mathcal{Y}\right):=\left\{\omega \in \mathcal{D}^{p, 1}\left(B^{n} \times \mathcal{Y}\right) \mid d_{y} \omega^{(1)}=0\right\} .
$$

Graphs of $\boldsymbol{B} \boldsymbol{V}$-maps. We introduce a class of $\mathcal{D}_{n, 1}$-currents associated to the graphs of $B V$-functions. To this aim, we observe that any form $\omega=\omega^{(1)} \in$ $\mathcal{D}^{n, 1}\left(B^{n} \times \mathcal{Y}\right)$ can be written as

$$
\omega^{(1)}=\sum_{i=1}^{n} \sum_{j=1}^{N}(-1)^{n-i} \phi_{i}^{j}(x, y) \widehat{d x^{i}} \wedge d y^{j}
$$

for some $\phi_{i}^{j} \in C_{0}^{\infty}\left(B^{n} \times \mathcal{Y}\right)$, and we will set $\phi^{j}:=\left(\phi_{1}^{j}, \ldots, \phi_{n}^{j}\right)$.

Definition 2.1. We say that a current $G \in \mathcal{D}_{n, 1}\left(B^{n} \times \mathcal{Y}\right)$ is in $B V-\operatorname{graph}\left(B^{n} \times \mathcal{Y}\right)$ if it decomposes into its absolutely continuous, Cantor, and Jump parts

$$
G:=G^{a}+G^{C}+G^{J},
$$

where $G_{(0)}^{C}=G_{(0)}^{J}=0$, and its action on forms in $\mathcal{D}^{n, 1}\left(B^{n} \times \mathcal{Y}\right)$ is given for any $\phi \in C_{c}^{\infty}\left(B^{n} \times \mathcal{Y}\right)$ by

$$
G(\phi(x, y) d x)=G^{a}(\phi(x, y) d x):=\int_{B^{n}} \phi(x, u(x)) d x
$$

for some function $u=u(G) \in B V\left(B^{n}, \mathcal{Y}\right)$ and, on forms $\omega=\omega^{(1)}$ satisfying (2.2), by

$$
\begin{aligned}
G^{a}\left(\omega^{(1)}\right) & :=\sum_{j=1}^{N} \int_{B^{n}}\left\langle\nabla u^{j}, \phi^{j}(x, u(x))\right\rangle d x \\
G^{C}\left(\omega^{(1)}\right) & :=\sum_{j=1}^{N} \int_{B^{n}} \phi^{j}(x, u(x)) d D^{C} u^{j} \\
G^{J}\left(\omega^{(1)}\right) & :=\sum_{j=1}^{N} \sum_{i=1}^{n} \int_{J_{u}}\left(\int_{\gamma_{x}} \phi_{i}^{j}(x, y) d y^{j}\right) v_{i} d \mathcal{H}^{n-1}(x),
\end{aligned}
$$

where $\gamma_{x}$ is a 1-dimensional integral chain in $\mathcal{Y}$ satisfying $\partial \gamma_{x}=\delta_{u^{+}(x)}-\delta_{u^{-}(x)}$ and $v=\left(v_{1}, \ldots, v_{n}\right)$ is the unit normal to $J_{u}$ at $x$, for $\mathcal{H}^{n-1}$-a.e. $x \in J_{u}$. 
Remark 2.2. If $n \geq 2$ in general the current $G$ has a non-zero boundary in $B^{n} \times$ $\mathcal{Y}$, even if $u \in W^{1,1}\left(B^{n}, \mathcal{Y}\right)$, i.e., if $G=G^{a}$. Take for example $n=2, \mathcal{Y}=S^{1} \subset$ $\mathbb{R}^{2}$, and $u(x)=x /|x|$, so that $G=G_{u}:=(\operatorname{Id} \bowtie u)_{\#} \llbracket B^{2} \rrbracket$ and hence

$$
\partial G\left\llcorner B^{2} \times S^{1}=-\delta_{0} \times \llbracket S^{1} \rrbracket,\right.
$$

where $\delta_{0}$ is the unit Dirac mass at the origin. However, as we shall see in Remark 6.10 below, the boundary $\partial G$ is null on every $(n-1)$-form $\widetilde{\omega}$ in $B^{n} \times \mathcal{Y}$ which has no "vertical" differentials.

Weak limits of smooth graphs. Let $\left\{u_{k}\right\} \subset C^{1}\left(B^{n}, \mathcal{Y}\right)$ be a sequence of smooth maps with equibounded $W^{1,1}$-energies, $\sup _{k}\left\|D u_{k}\right\|_{L^{1}}<\infty$. The currents $G_{u_{k}}$ carried by the graphs of the $u_{k}$ 's are well defined currents in $\mathcal{D}_{n, 1}\left(B^{n} \times \mathcal{Y}\right)$ with equibounded $\mathcal{E}_{1,1}$-norms. Therefore, possibly passing to a subsequence, we infer that $G_{u_{k}} \rightarrow T$ weakly in $\mathcal{D}_{n, 1}\left(B^{n} \times \mathcal{Y}\right)$ to some current $T \in \mathcal{D}_{n, 1}\left(B^{n} \times \mathcal{Y}\right)$, and $u_{k} \rightarrow u_{T}$ weakly in the $B V$-sense to some function $u_{T} \in B V\left(B^{n}, \mathcal{Y}\right)$. Therefore, we clearly have that

$$
T(\phi(x, y) d x)=\int_{B^{n}} \phi\left(x, u_{T}(x)\right) d x \quad \forall \phi \in C_{c}^{\infty}\left(B^{n} \times \mathcal{Y}\right) .
$$

Moreover, by lower semicontinuity we have $\|T\|_{\mathcal{E}_{1,1}}<\infty$ whereas, since the $G_{u_{k}}$ 's have no boundary in $B^{n} \times \mathcal{Y}$, by the weak convergence we also infer

$$
\partial T=0 \quad \text { on } \mathcal{Z}^{n-1,1}\left(B^{n} \times \mathcal{Y}\right) .
$$

Currents associated to graphs of $\boldsymbol{B} \boldsymbol{V}$-functions. Arguing as in Section 1, we associate to the weak limit current $T$ a current $G_{T} \in B V-\operatorname{graph}\left(B^{n} \times \mathcal{Y}\right)$, see Definition 2.1, where the function $u=u\left(G_{T}\right) \in B V\left(B^{n}, \mathcal{Y}\right)$ is given by $u_{T}$ and the $\gamma_{x}$ 's in the definition of the jump part $G_{T}^{J}$ are the indecomposable 1dimensional integral chains defined as in the previous section, but for $\mathcal{H}^{n-1}$-a.e. $x \in J_{u_{T}}$, since $\|T\|_{\mathcal{E}_{1,1}}<\infty$, compare (1.2) and Definition 2.8 below. In general $\partial G_{T}\left\llcorner B^{n} \times \mathcal{Y} \neq 0\right.$. However, setting

$$
S_{T}:=T-G_{T},
$$

we clearly have $S_{T}(\phi(x, y) d x)=0$ for every $\phi \in C_{c}^{\infty}\left(B^{n} \times \mathcal{Y}\right)$. Moreover, we also have:

Proposition 2.3. $S_{T}(\omega)=0$ for every form $\omega=\omega^{(1)}$ such that $\omega=d_{y} \widetilde{\omega}$ for some $\tilde{\omega} \in \mathcal{D}^{n-1,0}\left(B^{n} \times \mathcal{Y}\right)$.

Proof. Write $\widetilde{\omega}:=\omega_{\varphi} \wedge \eta$ for some $\eta \in C_{0}^{\infty}(\mathcal{Y})$ and $\varphi=\left(\varphi^{1}, \ldots, \varphi^{n}\right) \in$ $C_{0}^{\infty}\left(B^{n}, \mathbb{R}^{n}\right)$, where

$$
\omega_{\varphi}:=\sum_{i=1}^{n}(-1)^{i-1} \varphi^{i}(x) \widehat{d x^{i}}
$$


Since

$$
d\left(\omega_{\varphi} \wedge \eta\right)=\operatorname{div} \varphi(x) \eta(y) d x+(-1)^{n-1} \omega_{\varphi} \wedge d_{y} \eta
$$

and $T\left(d\left(\omega_{\varphi} \wedge \eta\right)\right)=\partial T\left(\omega_{\varphi} \wedge \eta\right)=0$, we have

$$
(-1)^{n} T(\operatorname{div} \varphi(x) \eta(y) d x)=T\left(\omega_{\varphi} \wedge d_{y} \eta\right),
$$

so that

$$
S_{T}\left(\omega_{\varphi} \wedge d_{y} \eta\right)=(-1)^{n} T(\operatorname{div} \varphi(x) \eta(y) d x)-G_{T}\left(\omega_{\varphi} \wedge d_{y} \eta\right)
$$

Moreover, since $T_{(0)}=G_{T(0)}$, by (2.3) we have

$$
T(\operatorname{div} \varphi(x) \eta(y) d x)=\int_{B^{n}} \operatorname{div} \varphi(x) \eta\left(u_{T}(x)\right) d x=-\left\langle D\left(\eta \circ u_{T}\right), \varphi\right\rangle
$$

whereas, taking $\phi_{i}^{j}=\varphi^{i} D_{y_{j}} \eta$ in (2.2), by the definition of $G_{T}$, since $\partial \gamma_{x}=$ $\delta_{u_{T}^{+}(x)}-\delta_{u_{T}^{-}(x)}$ we infer

$$
\begin{aligned}
(-1)^{n-1} G_{T}\left(\omega_{\varphi} \wedge d_{y} \eta\right)= & \sum_{j=1}^{N} \int_{B^{n}} \frac{\partial \eta}{\partial y^{j}}\left(u_{T}(x)\right)\left\langle\nabla u_{T}^{j}(x), \varphi(x)\right\rangle d x \\
& +\sum_{j=1}^{N} \int_{B^{n}} \frac{\partial \eta}{\partial y^{j}}\left(u_{T}(x)\right) \varphi(x) d D^{C} u_{T}^{j} \\
& +\int_{J_{u_{T}}}\left(\eta\left(u_{T}^{+}(x)\right)-\eta\left(u_{T}^{-}(x)\right)\langle\varphi(x), v(x)\rangle d \mathcal{H}^{n-1}\right.
\end{aligned}
$$

Finally, by the chain rule for the derivative $D\left(\eta \circ u_{T}\right)$ we obtain

$$
(-1)^{n-1} G_{T}\left(\omega_{\varphi} \wedge d_{y} \eta\right)=\left\langle D\left(\eta \circ u_{T}\right), \varphi\right\rangle
$$

and hence that $S_{T}\left(\omega_{\varphi} \wedge d_{y} \eta\right)=0$.

In conclusion, similarly to [14, Vol. II, Section 5.4.3], we infer that the weak limit current $T$ is given by

$$
T=G_{T}+S_{T}, \quad S_{T}=\sum_{s=1}^{\bar{s}} \mathbb{L}_{S}(T) \times \gamma_{s} \quad \text { on } \quad \mathcal{Z}^{n, 1}\left(B^{n} \times \mathcal{Y}\right)
$$

where $\mathbb{L}_{s}(T) \in \mathcal{D}_{n-1}\left(B^{n}\right)$ is defined by

$$
\mathbb{L}_{s}(T)=(-1)^{n-1} \pi^{\#}\left(S_{T}\left\llcorner\widehat{\pi}^{\#} \omega^{s}\right), \quad s=1, \ldots, \bar{s},\right.
$$

so that

$$
\mathbb{L}_{S}(T)(\phi)=S_{T}\left(\pi^{\#} \phi \wedge \widehat{\pi}^{\#} \omega^{s}\right) \quad \forall \phi \in \mathcal{D}^{n-1}\left(B^{n}\right) .
$$


Notice that by (2.4) we have

$$
\partial \mathbb{L}_{s}(T)\left\llcorner B^{n}=(-1)^{n-1} \pi_{\#}\left(\left(\partial G_{T}\right)\left\llcorner\widehat{\pi}^{\#} \omega^{s}\right) \quad \forall s=1, \ldots, \bar{s} .\right.\right.
$$

Finally, setting

$$
S_{T, \text { sing }}:=T-G_{T}-\sum_{s=1}^{\bar{s}} \mathbb{L}_{s}(T) \times \gamma_{s},
$$

see Remark 1.4, it turns out that $S_{T \text {,sing }}$ is nonzero only possibly on forms $\omega$ with non-zero vertical component, $\omega^{(1)} \neq 0$, and such that $d_{y} \omega^{(1)} \neq 0$.

Parametric polyconvex lower semicontinuous extension of the total variation. Following [14], Vol. II, Section 1.2, we recall that the parametric polyconvex lower semicontinuous extension $\|\cdot\|_{T V}$ of the total variation integrand of mappings from $B^{n}$ to $\mathbb{R}^{N}$ has the form

$$
\|\xi\|_{T V}:=\left|\xi_{(1)}\right| \quad \forall \xi \in \Lambda_{n} \mathbb{R}^{n+N} \quad \text { such that } \quad \xi^{\overline{0} 0} \geq 0,
$$

where $\xi^{\overline{0} 0}$ denotes the coefficient of the first component of any $n$-vector $\xi \in$ $\Lambda_{n} \mathbb{R}^{n+N}$ and $\left|\xi_{(1)}\right|$ is the euclidean norm of the component $\xi_{(1)}$ of $\xi$ in $\Lambda_{n-1} \mathbb{R}^{n} \otimes$ $\Lambda_{1} \mathbb{R}^{N}$. We have

Proposition 2.4. The parametric polyconvex lower semicontinuous extension $F(x, u, \xi): B^{n} \times \mathbb{R}^{N} \times \Lambda_{n} \mathbb{R}^{n+N} \rightarrow \overline{\mathbb{R}}^{+}$of the total variation integrand of mappings from $B^{n}$ into any smooth manifold $\mathcal{Y} \subset \mathbb{R}^{N}$ is given by

$$
F(x, u, \xi):= \begin{cases}\|\xi\|_{T V} & \text { if } u \in \mathcal{Y}, \xi \in \Lambda_{n}\left(\mathbb{R}^{n} \times T_{u} \mathcal{Y}\right) \\ +\infty & \text { otherwise, }\end{cases}
$$

where $\|\xi\|_{T V}$ is given by (2.9) and $T_{u} \mathcal{Y}$ is the tangent space to $\mathcal{Y}$ at $u$.

Parametric total variation. If $T \in \mathcal{D}_{n, 1}\left(B^{n} \times \mathcal{Y}\right)$ is such that $\|T\|_{\mathcal{E}_{1,1}}<\infty$, we denote by

$$
T=\|T\|_{\mathcal{E}_{1,1}}\llcorner\vec{T}
$$

the Radon-Nikodym decomposition of $T$ with respect to the $\mathcal{E}_{1,1}$-norm, $T$ being identified with the $\mathbb{R}^{1+N n}$-valued linear functional

$$
T:=\left(T^{\overline{0} 0},\left(T^{\bar{i} j}\right)_{\mathbb{R}^{N n}}\right), \quad i=1, \ldots n, \quad j=1, \ldots N,
$$

where

$$
T^{\overline{0} 0}(\phi):=T(\phi d x), \quad T^{\bar{i} j}(\phi):=T\left(\phi \widehat{d x^{i}} \wedge d y^{j}\right), \quad \phi \in C_{0}^{\infty}\left(B^{n} \times \mathcal{Y}\right) .
$$

Definition 2.5. The parametric variational integral associated to the total variation integral is defined for every Borel set $B \subset B^{n}$ by

$$
\mathcal{F}_{1,1}(T, B \times \mathcal{Y}):=\int_{B \times \mathcal{Y}} F(\pi(z), \widehat{\pi}(z), \vec{T}(z)) d\|T\|_{\mathcal{E}_{1,1}}(z)
$$

where $F(x, u, \xi)$ is given by (2.10), and we let $\mathcal{F}_{1,1}(T):=\mathcal{F}_{1,1}\left(T, B^{n} \times \mathcal{Y}\right)$. 
Gap phenomenon. If $T \in \mathcal{D}_{n, 1}\left(B^{n} \times \mathcal{Y}\right)$ is the weak limit of a sequence $\left\{G_{u_{k}}\right\}$ of graphs of smooth maps $\left\{u_{k}\right\} \subset C^{1}\left(B^{n}, \mathcal{Y}\right)$ with equibounded $W^{1,1}$-energies, since $\mathcal{F}_{1,1}\left(G_{u_{k}}\right)=\left\|D u_{k}\right\|_{L^{1}}$, by the lower semicontinuity of $\mathcal{F}_{1,1}$ with respect to the weak convergence in $\mathcal{D}_{n, 1}$ we infer that $\mathcal{F}_{1,1}(T)<\infty$. Moreover, if $T$ decomposes as in (2.6) on the whole of $\mathcal{D}^{n, 1}\left(B^{n} \times \mathcal{Y}\right)$, i.e., the singular part $S_{T, \text { sing }}$ defined in (2.8) vanishes, and if the $\mathbb{L}_{S}(T)$ 's are integer multiplicity rectifiable currents, an explicit formula can be obtained. However, similarly to the case of dimension $n=1$, a gap phenomenon occurs. More precisely, in general for every smooth sequence $\left\{u_{k}\right\} \subset C^{1}\left(B^{n}, \mathcal{Y}\right)$ such that $G_{u_{k}} \rightarrow T$ weakly in $\mathcal{D}_{n, 1}\left(B^{n} \times \mathcal{Y}\right)$ we have that

$$
\liminf _{k \rightarrow \infty} \mathcal{F}_{1,1}\left(G_{u_{k}}\right) \geq \mathcal{F}_{1,1}(T)+C
$$

for some absolute constant $C>0$, see Remark 1.5.

Vertical homology classes. As in Definition 1.6, we are therefore led to consider vertical homology equivalence classes of currents satisfying the same structure properties as weak limits of graphs of smooth maps $u_{k}: B^{n} \rightarrow \mathcal{Y}$ with equibounded total variation, $\sup _{k}\left\|D u_{k}\right\|_{L^{1}}<\infty$. More precisely, we say that

$$
T \sim \widetilde{T} \Longleftrightarrow T(\omega)=\widetilde{T}(\omega) \quad \forall \omega \in \mathcal{Z}^{n, 1}\left(B^{n} \times \mathcal{Y}\right) .
$$

Moreover, we will say that $T_{k} \rightarrow T$ weakly in $\mathcal{Z}_{n, 1}\left(B^{n} \times \mathcal{Y}\right)$ if $T_{k}(\omega) \rightarrow T(\omega)$ for every $\omega \in \mathcal{Z}^{n, 1}\left(B^{n} \times \mathcal{Y}\right)$.

Definition 2.6. We denote by $\mathcal{E}_{1,1}-\operatorname{graph}\left(B^{n} \times \mathcal{Y}\right)$ the set of equivalence classes, in the sense of (2.11), of currents $T$ in $\mathcal{D}_{n, 1}\left(B^{n} \times \mathcal{Y}\right)$ which have no interior boundary,

$$
\partial T=0 \quad \text { on } \quad \mathcal{Z}^{n-1,1}\left(B^{n} \times \mathcal{Y}\right),
$$

finite $\mathcal{E}_{1,1}$-norm, i.e.

$$
\|T\|_{\mathcal{E}_{1,1}}:=\sup \left\{T(\omega) \mid \omega \in \mathcal{Z}^{n, 1}\left(B^{n} \times \mathcal{Y}\right),\|\omega\|_{\mathcal{E}_{1,1}} \leq 1\right\}<\infty,
$$

and decompose as

$$
T=G_{T}+S_{T}, \quad S_{T}=\sum_{s=1}^{\bar{s}} \mathbb{L}_{s}(T) \times \gamma_{s} \quad \text { on } \quad \mathcal{Z}^{n, 1}\left(B^{n} \times \mathcal{Y}\right),
$$

where $G_{T} \in B V-\operatorname{graph}\left(B^{n} \times \mathcal{Y}\right)$, see Definition 2.1, and $\mathbb{L}_{s}(T)$ is an integer multiplicity rectifiable current in $\mathcal{R}_{n-1}\left(B^{n}\right)$ for everys.

Remark 2.7. If $\widetilde{T} \sim T$, in general $G_{\widetilde{T}} \neq G_{T}$. However, the corresponding $B V$ functions coincide, i.e., $u\left(G_{T}\right)=u\left(G_{\widetilde{T}}\right)$, see Definition 2.1. This yields that we may refer to the underlying functions $u_{T} \in B V\left(B^{n}, \mathcal{Y}\right)$ associated to currents $T$ in $\mathcal{E}_{1,1}-\operatorname{graph}\left(B^{n} \times \mathcal{Y}\right)$. 
Jump-concentration set. Moreover, if $\mathcal{L}(T)$ denotes the $(n-1)$-rectifiable set given by the union of the sets of positive multiplicity of the $\mathbb{L}_{s}(T)$ 's, we infer that the union

$$
J_{c}(T):=J_{u_{T}} \cup \mathcal{L}(T)
$$

does not depend on the choice of the representative in $T$. As in dimension one, the countably $\mathcal{H}^{n-1}$-rectifiable set $J_{c}(T)$ is said to be the set of points of jumpconcentration of $T$.

Restriction over points of jump-concentration. Let $T \in \mathcal{E}_{1,1}-\operatorname{graph}\left(B^{n} \times \mathcal{Y}\right)$ and let $v_{T}: J_{c}(T) \rightarrow S^{n-1}$ denote an extension to $J_{c}(T)$ of the unit normal $v_{u_{T}}$ to the Jump set $J_{u_{T}}$. For any $k=1, \ldots, n-1$, let $P$ be an oriented $k$ dimensional subspace in $\mathbb{R}^{n}$ and $P_{\lambda}:=P+\sum_{i=1}^{n-k} \lambda_{i} v_{i}$ the family of oriented $k$-planes parallel to $P$, where $\lambda:=\left(\lambda_{1}, \ldots, \lambda_{n-k}\right) \in \mathbb{R}^{n-k}, \operatorname{span}\left(v_{1}, \ldots, v_{n-k}\right)$ being the orthogonal space to $P$. Since $T$ has finite $\mathcal{E}_{1,1}$-norm, similarly to the case of normal currents, for $\mathcal{L}^{n-k}$-a.e. $\lambda$ such that $P_{\lambda} \cap B^{n} \neq \emptyset$, the slice $T\left\llcorner\pi^{-1}\left(P_{\lambda}\right)\right.$ of $T$ over $\pi^{-1}\left(P_{\lambda}\right)$ is a well defined $k$-dimensional current in $\mathcal{E}_{1,1}$-graph $\left(\left(B^{n} \cap\right.\right.$ $\left.P_{\lambda}\right) \times \mathcal{Y}$ ) with finite $\mathcal{E}_{1,1}$-norm. Moreover, for any such $\lambda$ we have

$$
J_{c}\left(T\left\llcorner\pi^{-1}\left(P_{\lambda}\right)\right)=J_{c}(T) \cap P_{\lambda} \quad \text { in the } \mathcal{H}^{k-1} \text {-a.e. sense },\right.
$$

whereas the $B V$-function associated to $T\left\llcorner\pi^{-1}\left(P_{\lambda}\right)\right.$ is equal to the restriction $u_{T \mid P_{\lambda}}$ of $u_{T}$ to $P_{\lambda}$. Therefore, in the particular case $k=1$, as in Section 1 the 1-dimensional restriction

$$
\widehat{\pi}_{\#}\left(\left(T\left\llcorner\pi^{-1}\left(P_{\lambda}\right)\right)\llcorner\{x\} \times \mathcal{Y}) \in \mathcal{D}_{1}(\mathcal{Y})\right.\right.
$$

of the 1-dimensional current $T\left\llcorner\pi^{-1}\left(P_{\lambda}\right)\right.$ over any point $x \in J_{C}(T) \cap P_{\lambda}$ such that $v_{T}(x)$ does not belong to $P$ is well defined. In this case, from the slicing properties of $B V$-functions, if $x \in\left(J_{c}(T) \backslash J_{u_{T}}\right) \cap P_{\lambda}$ we have $u_{T \mid P_{\lambda}}(x)=u_{T}(x)$. Moreover, if $x \in J_{u_{T}} \cap P_{\lambda}$, the one-sided approximate limits of $u_{T}$ are equal to the one-sided limits of the restriction $u_{T \mid P_{\lambda}}$, i.e.

$$
u_{T \mid P_{\lambda}}^{+}(x)=u_{T}^{+}(x) \text { and } u_{T \mid P_{\lambda}}^{-}(x)=u_{T}^{-}(x),
$$

provided that $\left\langle\nu, v_{u_{T}}(x)\right\rangle>0$, where $v$ is an orienting unit vector to $P$, compare Theorem 3.2. We finally infer that for $\mathcal{H}^{n-1}$-a.e. point $x \in J_{c}(T)$ the 1 dimensional restriction (2.13), up to the orientation, does not depend on the choice of the oriented 1-space $P$ and on $\lambda \in \mathbb{R}^{n-1}$, provided that $x \in P_{\lambda}$ and $v_{T}(x)$ does not belong to $P$. As a consequence we may and do give the following:

Definition 2.8. For $\mathcal{H}^{n-1}$-a.e. point $x \in J_{c}(T)$, the 1-dimensional restriction $\widehat{\pi}_{\#}(T\llcorner\{x\} \times \mathcal{Y})$ is well-defined by (2.13) for any oriented 1 -space $P$ and $\lambda \in$ $\mathbb{R}^{n-1}$ such that $x \in P_{\lambda}$ and $\left\langle\nu, \nu_{T}(x)\right\rangle>0$, where $v$ is the orienting unit vector to $P$. 
$\boldsymbol{B} \boldsymbol{V}$-energy. The gap phenomenon and the properties previously described lead us to define the $B V$-energy of a current $T \in \mathcal{E}_{1,1}-\operatorname{graph}\left(B^{n} \times \mathcal{Y}\right)$ as follows.

Definition 2.9. For $\mathcal{H}^{n-1}$-a.e. point $x \in J_{c}(T)$ we define $\Gamma_{T}(x)$ and $\mathcal{L}_{T}(x)$ by (1.5) and (1.6), respectively, where this time $\widehat{\pi}_{\#}(T\llcorner\{x\} \times \mathcal{Y})$ is the 1-dimensional restriction given by Definition 2.8 .

Definition 2.10. The $B V$-energy of a current $T \in \mathcal{E}_{1,1}-\operatorname{graph}\left(B^{n} \times \mathcal{Y}\right)$ is defined for every Borel set $B \subset B^{n}$ by

$$
\mathcal{E}_{1,1}(T, B \times \mathcal{Y}):=\int_{B}\left|\nabla u_{T}(x)\right| d x+\left|D^{C} u_{T}\right|(B)+\int_{J_{c}(T) \cap B} \mathcal{L}_{T}(x) d \mathcal{H}^{n-1}(x) .
$$

We also let

$$
\mathcal{E}_{1,1}(T):=\mathcal{E}_{1,1}\left(T, B^{n} \times \mathcal{Y}\right) .
$$

Of course, if $T=G_{u}$ is the current integration of $n$-forms in $\mathcal{D}^{n, 1}\left(B^{n} \times \mathcal{Y}\right)$ over the graph of a smooth $W^{1,1}$-function $u: B^{n} \rightarrow \mathcal{Y}$, then

$$
\mathcal{E}_{1,1}(u)=\mathcal{E}_{1,1}\left(G_{u}\right)=\|D u\|_{L^{1}} .
$$

Definition 2.11. We denote by cart ${ }^{1,1}\left(B^{n} \times \mathcal{Y}\right)$ the class of currents $T$ in $\mathcal{E}_{1,1^{-}}$ $\operatorname{graph}\left(B^{n} \times \mathcal{Y}\right)$ such that $\mathcal{E}_{1,1}(T)<\infty$.

Lower semicontinuity. Using the lower semicontinuity result in dimension $n=1$, see Theorem 1.7, and applying arguments as for instance in [7], in Section 3 we will prove in any dimension:

Theorem 2.12. Let $n \geq 2$ and $T \in \operatorname{cart}^{1,1}\left(B^{n} \times \mathcal{Y}\right)$. For every sequence of smooth maps $\left\{u_{k}\right\} \subset C^{1}\left(B^{n}, \mathcal{Y}\right)$ such that $G_{u_{k}} \rightarrow T$ weakly in $\mathcal{Z}_{n, 1}\left(B^{n} \times \mathcal{Y}\right)$, we have

$$
\liminf _{k \rightarrow \infty} \mathcal{E}_{1,1}\left(u_{k}\right) \geq \mathcal{E}_{1,1}(T) .
$$

A strong density result. In all the results stated below, we shall always assume that the first homotopy group $\pi_{1}(\mathcal{Y})$ is commutative. We shall prove in any dimension $n \geq 2$

Theorem 2.13. Let $T \in \operatorname{cart}^{1,1}\left(B^{n} \times \mathcal{Y}\right)$. There exists a sequence of smooth maps $\left\{u_{k}\right\} \subset C^{1}\left(B^{n}, \mathcal{Y}\right)$ such that $G_{u_{k}} \rightarrow T$ weakly in $\mathcal{Z}_{n, 1}\left(B^{n} \times \mathcal{Y}\right)$ and $\mathcal{E}_{1,1}\left(u_{k}\right) \rightarrow$ $\mathcal{E}_{1,1}(T)$ as $k \rightarrow \infty$.

More precisely, in Section 4 we will prove:

Theorem 2.14. Let $T \in \operatorname{cart}^{1,1}\left(B^{n} \times \mathcal{Y}\right)$. We can find a sequence of currents $\left\{T_{k}\right\} \subset \operatorname{cart}^{1,1}\left(B^{n} \times \mathcal{Y}\right)$ such that

$$
T_{k} \rightarrow T \text { weakly in } \mathcal{Z}_{n, 1}\left(B^{n} \times \mathcal{Y}\right), \quad \mathcal{E}_{1,1}\left(T_{k}\right) \rightarrow \mathcal{E}_{1,1}(T)
$$

and for all $k$ the corresponding function $u_{k}:=u_{T_{k}}$ in $B V\left(B^{n}, \mathcal{Y}\right)$ has no Cantor part, i.e, $\left|D^{C} u_{k}\right|=0$ for every $k$. Moreover, $u_{k}$ weakly converges to $u_{T}$ in the $B V$-sense and

$$
\lim _{k \rightarrow \infty}\left|D u_{k}\right|\left(B^{n}\right)=\left|D u_{T}\right|\left(B^{n}\right) .
$$


In Section 5 we will then prove

Theorem 2.15. Let $T \in \operatorname{cart}^{1,1}\left(B^{n} \times \mathcal{Y}\right)$ be such that the corresponding $B V$ function $u_{T} \in B V\left(B^{n}, \mathcal{Y}\right)$ has no Cantor part, i.e, $\left|D^{C} u_{T}\right|=0$. There exists a sequence of smooth maps $\left\{u_{k}\right\} \subset C^{1}\left(B^{n}, \mathcal{Y}\right)$ such that $G_{u_{k}} \rightarrow T$ weakly in $\mathcal{Z}_{n, 1}\left(B^{n} \times \mathcal{Y}\right)$ and the energy $\mathcal{E}_{1,1}\left(u_{k}\right) \rightarrow \mathcal{E}_{1,1}(T)$ as $k \rightarrow \infty$.

By a diagonal argument we then clearly obtain Theorem 2.13 .

Relaxed total variation functional. As a consequence, setting

$$
\begin{array}{r}
\widetilde{\mathcal{E}_{1,1}}(T):=\inf \left\{\liminf _{k \rightarrow \infty} \int_{B^{n}}\left|D u_{k}\right| d x:\left\{u_{k}\right\} \subset C^{1}\left(B^{n}, \mathcal{Y}\right),\right. \\
\left.G_{u_{k}} \rightarrow T \quad \text { weakly in } \mathcal{Z}_{n, 1}\left(B^{n} \times \mathcal{Y}\right)\right\},
\end{array}
$$

by Theorems 2.12 and 2.13 we conclude that

$$
\mathcal{E}_{1,1}(T)=\widetilde{\mathcal{E}_{1,1}}(T) \quad \forall T \in \operatorname{cart}^{1,1}\left(B^{n} \times \mathcal{Y}\right) .
$$

Properties. By Theorems 2.12 and 2.13 we readily infer the following lower semicontinuity result.

Proposition 2.16. Let $\left\{T_{k}\right\} \subset \operatorname{cart}^{1,1}\left(B^{n} \times \mathcal{Y}\right)$ converge weakly in $\mathcal{Z}_{n, 1}\left(B^{n} \times \mathcal{Y}\right)$, $T_{k} \rightarrow T$, to some $T \in \operatorname{cart}^{1,1}\left(B^{n} \times \mathcal{Y}\right)$. Then

$$
\mathcal{E}_{1,1}(T) \leq \liminf _{k \rightarrow \infty} \mathcal{E}_{1,1}\left(T_{k}\right) .
$$

As a consequence of Theorem 2.13, in the final part of this section we prove that the class of Cartesian currents cart ${ }^{1,1}\left(B^{n} \times \mathcal{Y}\right)$ is closed under weak convergence with equibounded energies.

Theorem 2.17. Let $\left\{T_{k}\right\} \subset$ cart $^{1,1}\left(B^{n} \times \mathcal{Y}\right)$ converge weakly in $\mathcal{Z}_{n, 1}\left(B^{n} \times \mathcal{Y}\right)$, $T_{k} \rightarrow T$, to some $T \in \mathcal{D}_{n, 1}\left(B^{n} \times \mathcal{Y}\right)$, and $\sup _{k} \mathcal{E}_{1,1}\left(T_{k}\right)<\infty$. Then $T \in$ $\operatorname{cart}^{1,1}\left(B^{n} \times \mathcal{Y}\right)$.

By the relative compactness of $\mathcal{E}_{1,1}$-bounded sets in $\mathcal{D}_{n, 1}\left(B^{n} \times \mathcal{Y}\right)$, we then readily infer the following compactness property.

Proposition 2.18. Let $\left\{T_{k}\right\} \subset$ cart $^{1,1}\left(B^{n} \times \mathcal{Y}\right)$ be such that $\sup _{k} \mathcal{E}_{1,1}\left(T_{k}\right)<\infty$. Then, possibly passing to a subsequence, $T_{k} \rightarrow T$ weakly in $\mathcal{Z}_{n, 1}\left(B^{n} \times \mathcal{Y}\right)$ to some $T \in \operatorname{cart}^{1,1}\left(B^{n} \times \mathcal{Y}\right)$.

Proof of Theorem 2.17. By Theorem 2.13, and by a diagonal procedure, we may and will assume that $T_{k}=G_{u_{k}}$ for some smooth sequence $\left\{u_{k}\right\} \subset C^{1}\left(B^{n}, \mathcal{Y}\right)$. As a consequence, by the first part of this section we infer that $T$ satisfies (2.4) and 
(2.6). It then remains to show that the $\mathbb{L}_{s}(T)$ 's in (2.6) are integer multiplicity rectifiable current in $\mathcal{R}_{n-1}\left(B^{n}\right)$. In this case, in fact, since $\|T\|_{\mathcal{E}_{1,1}}<\infty$, we obtain that $T \in \mathcal{E}_{1,1}$-graph $\left(B^{n} \times \mathcal{Y}\right)$, see Definition 2.6, and hence, by lower semicontinuity, Theorem 2.12, and the condition $\sup _{k} \mathcal{E}_{1,1}\left(G_{u_{k}}\right)<\infty$, we conclude that $\mathcal{E}_{1,1}(T)<\infty$, which yields $T \in \operatorname{cart}^{1,1}\left(B^{n} \times \mathcal{Y}\right)$, according to Definition 2.11. To prove that the $\mathbb{L}_{s}(T)$ 's are integer multiplicity rectifiable currents we make use of the following slicing argument.

As before, let $P$ be an oriented 1-space in $\mathbb{R}^{n}$ and $\left\{P_{\lambda}\right\}_{\lambda \in \mathbb{R}^{n-1}}$ the family of oriented straight lines parallel to $P$. For $\mathcal{H}^{n-1}$-a.e. $\lambda$ the slice $T\left\llcorner\pi^{-1}\left(P_{\lambda}\right)\right.$ of $T$ over $\pi^{-1}\left(P_{\lambda}\right)$ is well defined on $\mathcal{Z}^{1,1}\left(\left(B^{n} \cap P_{\lambda}\right) \times \mathcal{Y}\right)$ and $G_{u_{k}}\left\llcorner\pi^{-1}\left(P_{\lambda}\right)\right.$ belongs to cart ${ }^{1,1}\left(\left(B^{n} \cap P_{\lambda}\right) \times \mathcal{Y}\right)$ for every $k$. Moreover, since $G_{u_{k}} \rightarrow T$ weakly in $\mathcal{Z}_{n, 1}$, for $\mathcal{H}^{n-1}$-a.e. $\lambda$, passing to a subsequence we have $G_{u_{k}}\left\llcorner\pi^{-1}\left(P_{\lambda}\right) \rightarrow\right.$ $T\left\llcorner\pi^{-1}\left(P_{\lambda}\right)\right.$ weakly in $\mathcal{Z}_{1,1}\left(\left(B^{n} \cap P_{\lambda}\right) \times \mathcal{Y}\right)$, with $\sup _{k} \mathbf{M}\left(G_{u_{k}}\left\llcorner\pi^{-1}\left(P_{\lambda}\right)\right)<\infty\right.$, so that by the closure-compactness of cart ${ }^{1,1}$ on 1-dimensional domains, we infer that $T\left\llcorner\pi^{-1}\left(P_{\lambda}\right) \in \operatorname{cart}^{1,1}\left(\left(B^{n} \cap P_{\lambda}\right) \times \mathcal{Y}\right)\right.$.

Therefore, the 0 -dimensional slices $\mathbb{L}_{s}(T)\left\llcorner\pi^{-1}\left(P_{\lambda}\right)\right.$ are rectifiable in $\mathcal{R}_{0}\left(B^{n} \cap\right.$ $\left.P_{\lambda}\right)$, as $T\left\llcorner\pi^{-1}\left(P_{\lambda}\right)\right.$ belongs to cart ${ }^{1,1}\left(\left(B^{n} \cap P_{\lambda}\right) \times \mathcal{Y}\right)$ and $\mathbb{L}_{s}(T)\left\llcorner\pi^{-1}\left(P_{\lambda}\right)=\right.$ $\mathbb{L}_{s}\left(T\left\llcorner\pi^{-1}\left(P_{\lambda}\right)\right)\right.$. Since the $\mathbb{L}_{s}(T)$ 's are flat chains, see Lemma 2.19 below, arguing as in [12], by White's rectifiability criterion [23], see also [3], we infer that $\mathbb{L}_{s}(T)$ is an integer multiplicity rectifiable current in $\mathcal{R}_{n-1}\left(B^{n}\right)$ for every $s$, as required.

Lemma 2.19. The $\mathbb{L}_{s}(T)$ 's are flat chains in $B^{n}$.

Proof. By Theorem 2.13, we may and will assume that $T$ is the weak limit of $G_{u_{k}}$ for some smooth sequence $\left\{u_{k}\right\} \subset C^{1}\left(B^{n}, \mathcal{Y}\right)$ such that $\sup _{k}\left\|u_{k}\right\|_{W^{1,1}}<\infty$. The proof follows the same lines as the proof of [17, Theorem 2.15]. Since $u_{k} \in$ $B V\left(B^{n}, \mathcal{Y}\right)$ is smooth, for all $k$ and $s$ we infer that $\mathbb{L}_{s}\left(G_{u_{k}}\right):=\pi_{\#}\left(G_{u_{k}}\left\llcorner\widehat{\pi}^{\#} \omega^{s}\right)\right.$ is a flat chain with equibounded flat norms. Recall that the flat norm $\mathbf{F}\left(\mathbb{L}_{s}\left(G_{u_{k}}\right)\right)$ of $\mathbb{L}_{s}\left(G_{u_{k}}\right)$ is given by

$$
\mathbf{F}\left(\mathbb{L}_{s}\left(G_{u_{k}}\right)\right):=\sup \left\{\mathbb{L}_{s}\left(G_{u_{k}}\right)(\phi) \mid \phi \in \mathcal{D}^{n-1}\left(B^{n}\right), \mathbf{F}(\phi) \leq 1\right\},
$$

where

$$
\mathbf{F}(\phi):=\max \left\{\sup _{x \in B^{n}}\|\phi(x)\|, \sup _{x \in B^{n}}\|d \phi(x)\|\right\} .
$$

Next, since $u_{k} \rightarrow u_{T}$ weakly in the $B V$-sense, we deduce that $\left\{\mathbb{L}_{s}\left(G_{u_{k}}\right)(\phi)\right\}_{k}$ is a Cauchy sequence for every $\phi$ such that $\mathbf{F}(\phi) \leq 1$. If $\mathcal{F}^{n-1}\left(B^{n}\right)$ denotes a countable dense subset of smooth forms $\phi$ in $\mathcal{D}^{n-1}\left(B^{n}\right)$ satisfying $\mathbf{F}(\phi) \leq 1$, by a diagonal argument we infer that

$$
\sup \left\{\left(\mathbb{L}_{s}\left(G_{u_{k}}\right)-\mathbb{L}_{s}\left(G_{u_{h}}\right)\right)(\phi) \mid \phi \in \mathcal{F}^{n-1}\left(B^{n}\right)\right\}
$$

is small for $k, h$ large. This yields that $\left\{\mathbb{L}_{s}\left(G_{u_{k}}\right)\right\}_{k}$ is a Cauchy sequence with respect to the flat norm, i.e., that

$$
\mathbf{F}\left(\mathbb{L}_{s}\left(G_{u_{k}}\right)-\mathbb{L}_{s}\left(G_{u_{h}}\right)\right):=\sup \left\{\left(\mathbb{L}_{s}\left(G_{u_{k}}\right)-\mathbb{L}_{s}\left(G_{u_{h}}\right)\right)(\phi) \mid \phi \in \mathcal{D}^{n-1}\left(B^{n}\right), \mathbf{F}(\phi) \leq 1\right\}
$$


is small for $k, h$ large and therefore, due to weak convergence of $G_{u_{k}}$ to $T$, that $R_{S}:=\pi_{\#}\left(T\left\llcorner\widehat{\pi}^{\#} \omega^{s}\right)\right.$ is a flat chain. Similarly, by using a trivial extension of Theorem 6.7 below, we infer that $D_{s}:=\pi_{\#}\left(G_{T}\left\llcorner\widehat{\pi}^{\#} \omega^{s}\right)\right.$ is a flat chain and hence, since $(-1)^{n-1} \mathbb{L}_{s}(T)=R_{S}-D_{s}$, compare (2.6) and (2.7), we conclude that $\mathbb{L}_{S}(T)$ is a flat chain, too.

\section{Lower semicontinuity}

In this section we prove Theorem 2.12, by recovering it from the one dimensional case. To this aim, we recall the following properties from $B V$-functions theory, compare [2, Section 3.11].

One-dimensional restrictions of $\boldsymbol{B} \boldsymbol{V}$-functions. Let $\Omega \subset \mathbb{R}^{n}$ be an open set. Given $v \in S^{n-1}$ we denote by $\pi_{v}$ the hyperplane in $\mathbb{R}^{n}$ orthogonal to $v$ and by $\Omega_{v}$ the orthogonal projection of $\Omega$ on $\pi_{v}$. For any $y \in \Omega_{v}$ we let

$$
\Omega_{y}^{v}:=\{t \in \mathbb{R} \mid y+t v \in \Omega\}
$$

denote the (non-empty) section of $\Omega$ corresponding to $y$. Accordingly, for any function $u: B \subset \Omega \rightarrow \mathbb{R}^{N}$ and any $y \in B_{v}$ the function $u_{y}^{v}: B_{y}^{v} \rightarrow \mathbb{R}^{N}$ is defined by

$$
u_{y}^{v}(t):=u(y+t v)
$$

Proposition 3.1. Let $u \in L^{1}\left(\Omega, \mathbb{R}^{N}\right)$. Then $u \in B V\left(\Omega, \mathbb{R}^{N}\right)$ if and only if there exist $n$ linearly independent unit vectors $v_{i}$ such that $u_{y}^{v_{i}} \in B V\left(\Omega_{y}^{v_{i}}, \mathbb{R}^{N}\right)$ for $\mathcal{L}^{n-1}$-a.e. $y \in \Omega_{v_{i}}$ and

$$
\int_{\Omega_{v_{i}}}\left|D u_{y}^{v_{i}}\right|\left(\Omega_{y}^{v_{i}}\right) d \mathcal{L}^{n-1}(y)<\infty \quad \forall i=1, \ldots, n .
$$

Theorem 3.2. If $u \in B V\left(\Omega, \mathbb{R}^{N}\right)$ and $v \in S^{n-1}$, then

$$
\begin{array}{ll}
\langle D u, v\rangle=\mathcal{L}^{n-1}\left\llcorner\Omega_{v} \otimes D u_{y}^{v},\right. & \left\langle D^{a} u, v\right\rangle=\mathcal{L}^{n-1}\left\llcorner\Omega_{v} \otimes D^{a} u_{y}^{v},\right. \\
\left\langle D^{J} u, v\right\rangle=\mathcal{L}^{n-1}\left\llcorner\Omega_{v} \otimes D^{J} u_{y}^{v},\right. & \left\langle D^{C} u, v\right\rangle=\mathcal{L}^{n-1}\left\llcorner\Omega_{v} \otimes D^{C} u_{y}^{v} .\right.
\end{array}
$$

In addition, for $\mathcal{L}^{n-1}$-a.e. $y \in \Omega_{v}$ the precise representative $u^{*}$ has classical directional derivatives along $v \mathcal{L}^{1}$-a.e. in $\Omega_{y}^{v}$, the function $\left(u^{*}\right)_{y}^{v}$ is a good representative in the equivalence class of $u_{y}^{v}$, its Jump set is $\left(J_{u}\right)_{y}^{v}$ and

$$
\frac{\partial u^{*}}{\partial v}(y+t v)=\langle\nabla u(y+t v), v\rangle \quad \text { for } \mathcal{L}^{1} \text {-a.e. } t \in \Omega_{y}^{v} .
$$

Finally, $\sigma(t):=\left\langle\nu, v_{u}(y+t v)\right\rangle \neq 0$ for $\mathcal{L}^{n-1}$-a.e. $y \in \Omega_{v}$ and $\mathcal{L}^{1}$-a.e. $t \in \Omega_{y}^{v}$, and

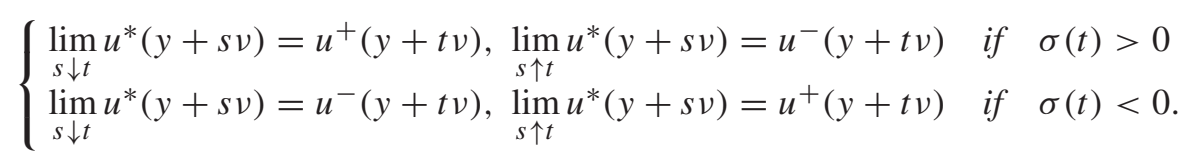


One-dimensional restrictions of Cartesian currents. If $T \in \operatorname{cart}^{1,1}\left(B^{n}, \mathcal{Y}\right)$, taking $\Omega=B^{n}$, for any $v \in S^{n-1}$ the 1-dimensional slice

$$
T_{y}^{v}:=T\left\llcorner\left(B^{n}\right)_{y}^{v} \times \mathcal{Y}\right.
$$

defines a Cartesian current $T_{y}^{v} \in \operatorname{cart}^{1,1}\left(\left(B^{n}\right)_{y}^{v} \times \mathcal{Y}\right)$ for $\mathcal{L}^{n-1}$-a.e. $\quad y \in\left(B^{n}\right)_{v}$. Also, by Theorem 3.2 and by Definition 2.10, we infer that the $B V$-energy of $T_{y}^{v}$ is given for $\mathcal{L}^{n-1}$-a.e. $y \in\left(B^{n}\right)_{v}$ by

$$
\begin{aligned}
\mathcal{E}_{1,1}\left(T_{y}^{v}, A_{y}^{v} \times \mathcal{Y}\right)= & \int_{A_{y}^{v}}\left|\left\langle\nabla u_{T}(y+t v), v\right\rangle\right| d t+\left|D^{C}\left(u_{T}\right)_{y}^{v}\right|\left(A_{y}^{v}\right) \\
& +\sum_{t \in\left(J_{c}(T) \cap A\right)_{y}^{v}} \mathcal{L}_{T}(y+t v)
\end{aligned}
$$

for any open set $A \subset B^{n}$.

Proof of Theorem 2.12. We follow [2, Theorem 5.4], [7]. Since $\left\{u_{k}\right\} \subset C^{1}\left(B^{n}, \mathcal{Y}\right)$ is such that $G_{u_{k}} \rightarrow T$ weakly in $\mathcal{Z}_{n, 1}\left(B^{n} \times \mathcal{Y}\right)$, for $\mathcal{L}^{n-1}$-a.e. $y \in\left(B^{n}\right)_{\nu}$ we infer that

$$
\left(G_{u_{k}}\right)_{y}^{v} \rightarrow T_{y}^{v} \quad \text { weakly in } \quad \mathcal{Z}_{1,1}\left(\left(B^{n}\right)_{y}^{v} \times \mathcal{Y}\right),
$$

where

$$
\left(G_{u_{k}}\right)_{y}^{v}=G_{\left(u_{k}\right)_{y}^{v}}, \quad\left(u_{k}\right)_{y}^{v}(t):=u_{k}(y+t v) \in C^{1}\left(\left(B^{n}\right)_{y}^{v}, \mathcal{Y}\right) .
$$

Therefore, arguing as in the proof of Theorem 1.7, we readily infer that

$$
\mathcal{E}_{1,1}\left(T_{y}^{v}, A_{y}^{v} \times \mathcal{Y}\right) \leq \liminf _{k \rightarrow \infty} \mathcal{E}_{1,1}\left(\left(u_{k}\right)_{y}^{v}, A_{y}^{v}\right)
$$

for any open set $A \subset B^{n}$, where

$$
\mathcal{E}_{1,1}\left(\left(u_{k}\right)_{y}^{v}, A_{y}^{v}\right)=\mathcal{E}_{1,1}\left(G_{\left(u_{k}\right) y}, A_{y}^{v} \times \mathcal{Y}\right)=\int_{A_{y}^{v}}\left|\left\langle\nabla u_{k}(y+t v), v\right\rangle\right| d t .
$$

We now denote by $\nu_{T}$ an extension to the countably $\mathcal{H}^{n-1}$-rectifiable set $J_{c}(T)$ of the outward unit normal to the Jump set $J_{u_{T}}$. By the coarea formula, for any $v \in S^{n-1}$ and any open set $A \subset B^{n}$, we have

$$
\int_{J_{c}(T) \cap A}\left|\left\langle v_{T}(x), v\right\rangle\right| f(x) d \mathcal{H}^{n-1}(x)=\int_{\pi_{v}} \sum_{t \in\left(J_{c}(T) \cap A\right)_{y}^{v}} f(y+t v) d \mathcal{L}^{n-1}(y)
$$

for any Borel function $f: J_{c}(T) \cap A \rightarrow[0,+\infty]$. Moreover, Theorem 3.2 gives

$$
\begin{aligned}
\int_{A}\left|\left\langle\nabla u_{T}, v\right\rangle\right| d x & =\int_{\pi_{v}}\left(\int_{A_{y}^{v}}\left|\nabla\left(u_{T}\right)_{y}^{v}(t)\right| d t\right) \mathcal{L}^{n-1}(y) \\
\left|\left\langle D^{C} u_{T}, v\right\rangle\right|(A) & =\int_{\pi_{v}}\left|D^{C}\left(u_{T}\right)_{y}^{v}\right|\left(A_{y}^{v}\right) d \mathcal{L}^{n-1}(y) .
\end{aligned}
$$


Therefore, setting for every open set $A \subset B^{n}$ and $v \in S^{n-1}$

$$
\begin{aligned}
\mathcal{E}_{1,1}(T, A \times \mathcal{Y}, v):= & \int_{A}\left|\left\langle\nabla u_{T}, v\right\rangle\right| d x+\left|\left\langle D^{C} u_{T}, v\right\rangle\right|(A) \\
& +\int_{J_{c}(T) \cap A}\left|\left\langle v_{T}(x), v\right\rangle\right| \mathcal{L}_{T}(x) d \mathcal{H}^{n-1}(x),
\end{aligned}
$$

by (3.1) we obtain the identity

$$
\mathcal{E}_{1,1}(T, A \times \mathcal{Y}, v)=\int_{\pi_{v}} \mathcal{E}_{1,1}\left(T_{y}^{v}, A_{y}^{v} \times \mathcal{Y}\right) d \mathcal{L}^{n-1}(y)
$$

Similarly, for every $k$ we obtain

$$
\mathcal{E}_{1,1}\left(u_{k}, A, v\right):=\int_{A}\left|\left\langle\nabla u_{k}, v\right\rangle\right| d x=\int_{\pi_{v}} \mathcal{E}_{1,1}\left(\left(u_{k}\right)_{y}^{v}, A_{y}^{v}\right) d \mathcal{L}^{n-1}(y) .
$$

We also notice that

$$
\mathcal{E}_{1,1}(T, A \times \mathcal{Y}, v) \leq \mathcal{E}_{1,1}(T, A \times \mathcal{Y}) \quad \text { and } \quad \mathcal{E}_{1,1}\left(u_{k}, A, v\right) \leq \mathcal{E}_{1,1}\left(u_{k}, A\right)
$$

Since

$$
\lim _{k \rightarrow \infty} \int_{\pi_{\nu}}\left(\int_{A_{y}^{v}}\left|\left(u_{k}\right)_{y}^{v}-\left(u_{T}\right)_{y}^{v}\right| d t\right) d \mathcal{L}^{n-1}(y)=\lim _{k \rightarrow \infty} \int_{A}\left|u_{k}-u_{T}\right| d x=0,
$$

we can find a sequence $\{k(h)\}$ such that

$$
\liminf _{k \rightarrow \infty} \mathcal{E}_{1,1}\left(u_{k}, A, v\right)=\lim _{h \rightarrow \infty} \mathcal{E}_{1,1}\left(u_{k(h)}, A, v\right)
$$

and $\left(G_{u_{k(h)}}\right)_{y}^{v}$ converges to $T_{y}^{v}$ weakly in $\mathcal{Z}_{1,1}\left(A_{y}^{v} \times \mathcal{Y}\right)$ as $h \rightarrow \infty$ for $\mathcal{L}^{n-1}$ a.e. $y \in \pi_{v}$. The lower semicontinuity property in dimension one, see (3.2), implies then

$$
\liminf _{h \rightarrow \infty} \mathcal{E}_{1,1}\left(\left(u_{k(h)}\right)_{y}^{v}, A_{y}^{v}\right) \geq \mathcal{E}_{1,1}\left(T_{y}^{v}, A_{y}^{v} \times \mathcal{Y}\right)
$$

for $\mathcal{L}^{n-1}$-a.e. $y \in \pi_{v}$. Integrating both sides on $\pi_{\nu}$, using Fatou's lemma and (3.3), (3.4), we get

$$
\liminf _{k \rightarrow \infty} \mathcal{E}_{1,1}\left(u_{k}, A, v\right)=\lim _{h \rightarrow \infty} \mathcal{E}_{1,1}\left(u_{k(h)}, A, v\right) \geq \mathcal{E}_{1,1}(T, A \times \mathcal{Y}, v) .
$$

Let $\lambda:=\mathcal{L}^{n}+\mathcal{L}_{T}(\cdot) \mathcal{H}^{n-1}\left\llcorner J_{c}(T)+\left|D^{C} u_{T}\right|\right.$ and let $\left\{v_{i}\right\} \subset S^{n-1}$ be a countable dense sequence. Choosing an $\mathcal{L}^{n}$-negligible set $E \subset B^{n} \backslash J_{c}(T)$ on which $\left|D^{C} u_{T}\right|$ is concentrated, we can define

$$
\varphi_{i}(x):=\left\{\begin{array}{lll}
\left|\left\langle\nabla u_{T}(x), v_{i}\right\rangle\right| & \text { if } & x \in B^{n} \backslash\left(E \cup J_{c}(T)\right) \\
\left|\left\langle v_{T}(x), v_{i}\right\rangle\right| \mathcal{L}_{T}(x) & \text { if } & x \in J_{c}(T) \\
\frac{\left|\left\langle D^{C} u_{T}, v_{i}\right\rangle\right|}{\left|D^{C} u_{T}\right|}(x) & \text { if } & x \in E
\end{array}\right.
$$


and obtain from (3.3) that

$$
\liminf _{k \rightarrow \infty} \mathcal{E}_{1,1}\left(u_{k}, A\right) \geq \liminf _{k \rightarrow \infty} \mathcal{E}_{1,1}\left(u_{k}, A, v_{i}\right) \geq \mathcal{E}_{1,1}\left(T, A \times \mathcal{Y}, v_{i}\right)=\int_{A} \varphi_{i} d \lambda
$$

for any $i \in \mathbb{N}$ and any open set $A \subset B^{n}$. By the superadditivity of the liminf operator, we obtain that

$$
\liminf _{k \rightarrow \infty} \mathcal{E}_{1,1}\left(u_{k}, B^{n}\right) \geq \sum_{i} \int_{A_{i}} \varphi_{i} d \lambda
$$

for any finite family of pairwise disjoint open sets $A_{i} \subset B^{n}$. We now recall that by [2, Lemma 2.35]

$$
\int_{B^{n}} \sup _{i \in \mathbb{N}} \varphi_{i} d \lambda=\sup \left\{\sum_{i \in I} \int_{A_{i}} \varphi_{i} d \lambda\right\}
$$

where the supremum is taken over all finite sets $I \subset \mathbb{N}$ and all families $\left\{A_{i}\right\}_{i \in I}$ of pairwise disjoint open sets with compact closure in $B^{n}$. We then conclude that

$$
\begin{aligned}
\liminf _{k \rightarrow \infty} \mathcal{E}_{1,1}\left(u_{k}, B^{n}\right) & \geq \int_{B^{n}} \sup _{i \in \mathbb{N}} \varphi_{i} d \lambda \\
& =\int_{B^{n}}\left|\nabla u_{T}(x)\right| d x+\left|D^{C} u_{T}\right|\left(B^{n}\right)+\int_{J_{c}(T)} \mathcal{L}_{T}(x) d \mathcal{H}^{n-1}(x) \\
& =\mathcal{E}_{1,1}\left(T, B^{n} \times \mathcal{Y}\right) .
\end{aligned}
$$

\section{The density theorem: part I}

In this section we prove Theorem 2.14. To this aim we first recall that every $T \in$ $\operatorname{cart}^{1,1}\left(B^{n} \times \mathcal{Y}\right)$ decomposes as

$$
T=G_{T}+S_{T}, \quad S_{T}=\sum_{s=1}^{\bar{s}} \mathbb{L}_{s}(T) \times \gamma_{s} \quad \text { on } \quad \mathcal{Z}^{n, 1}\left(B^{n} \times \mathcal{Y}\right),
$$

see Definition 2.11. Let $u=u_{T} \in B V\left(B^{n}, \mathcal{Y}\right)$ be the $B V$-function associated to $T$, according to Remark 2.7. For every Borel set $B \subset B^{n}$ we have

$$
\mathcal{E}_{1,1}(T, B \times \mathcal{Y})=\int_{B}|\nabla u(x)| d x+\left|D^{C} u\right|(B)+\int_{J_{c}(T) \cap B} \mathcal{L}_{T}(x) d \mathcal{H}^{n-1}(x),
$$

where $J_{c}(T), \Gamma_{T}(x)$, and $\mathcal{L}_{T}(x)$ are given by (2.12), (1.5), and (1.6), respectively, compare Definition 2.10.

Slicing properties. Similarly to the case of normal currents, for every point $x_{0} \in$ $B^{n}$ and for a.e. radius $r \in\left(0, r_{0}\right)$, where $2 r_{0}:=\operatorname{dist}\left(x_{0}, \partial B^{n}\right)$, the slice

$$
\left\langle T, d_{x_{0}}, r\right\rangle=\left\langle G_{T}, d_{x_{0}}, r\right\rangle+\left\langle S_{T}, d_{x_{0}}, r\right\rangle,
$$


where $d_{x_{0}}(x, y):=\left|x-x_{0}\right|$, is a well-defined Cartesian current in $\operatorname{cart}^{1,1}\left(\partial B_{r}\left(x_{0}\right) \times\right.$ $\mathcal{Y})$. More precisely, let $u_{\left(r, x_{0}\right)}:=u_{\mid \partial B_{r}\left(x_{0}\right)}$ be the restriction of $u$ to $\partial B_{r}\left(x_{0}\right)$, which is a function in $B V\left(\partial B_{r}\left(x_{0}\right), \mathcal{Y}\right)$ with jump set satisfying $J_{u_{\left(r, x_{0}\right)}}=J_{u} \cap \partial B_{r}\left(x_{0}\right)$ in the $\mathcal{H}^{n-1}$-a.e. sense. The slice $\left\langle G_{T}, d_{x_{0}}, r\right\rangle$ is an $(n-1)$-dimensional current in $B V-\operatorname{graph}\left(\partial B_{r}\left(x_{0}\right) \times \mathcal{Y}\right)$ such that its action on forms in $\mathcal{D}^{n-1,1}\left(\partial B_{r}\left(x_{0}\right) \times \mathcal{Y}\right)$, according to a straightforward extension of Definition 2.1, depends on the restriction $u_{\left(r, x_{0}\right)}$ and on the 1-dimensional integral chains $\gamma_{x}$ in $\mathcal{Y}$ associated to the current $G_{T} \in B V-\operatorname{graph}\left(B^{n} \times \mathcal{Y}\right)$, so that in particular $\partial \gamma_{x}=\delta_{u_{\left(r, x_{0}\right)}^{+}(x)}-\delta_{u_{\left(r, x_{0}\right)}^{-}(x)}$ for $\mathcal{H}^{n-1}$-a.e. $x \in J_{u_{\left(r, x_{0}\right)}}$. Also,

$$
\left\langle S_{T}, d_{x_{0}}, r\right\rangle=\sum_{s=1}^{\bar{s}}\left\langle\mathbb{L}_{s}(T), \delta_{x_{0}}, r\right\rangle \times \gamma_{s} \quad \text { on } \quad \mathcal{Z}^{n-1,1}\left(\partial B_{r}\left(x_{0}\right) \times \mathcal{Y}\right),
$$

where $\delta_{x_{0}}(x):=\left|x-x_{0}\right|$. Finally, letting

$$
J_{c}\left(\left\langle T, d_{x_{0}}, r\right\rangle\right):=J_{u_{\left(r, x_{0}\right)}} \cup \mathcal{L}\left(\left\langle T, d_{x_{0}}, r\right\rangle\right),
$$

where $\mathcal{L}\left(\left\langle T, d_{x_{0}}, r\right\rangle\right)$ denotes the $(n-2)$-rectifiable set given by the union of the sets of positive multiplicity of the $\left\langle\mathbb{L}_{s}(T), \delta_{x_{0}}, r\right\rangle$ 's, we have, in the $\mathcal{H}^{n-1}$-a.e. sense,

$$
J_{c}\left(\left\langle T, d_{x_{0}}, r\right\rangle\right)=J_{c}(T) \cap \partial B_{r}\left(x_{0}\right),
$$

where $J_{c}(T)$ is given by (2.12). In this case we say that $r$ is a good radius for $T$ at $x_{0}$. Moreover, by the argument preceding Definition 2.8, we also infer that for any good radius

$$
\mathcal{L}_{\left\langle T, d_{x_{0}}, r\right\rangle}(x)=\mathcal{L}_{T}(x) \quad \text { for } \mathcal{H}^{n-1} \text {-a.e. } x \in J_{c}\left(\left\langle T, d_{x_{0}}, r\right\rangle\right)
$$

As a consequence, according to Definition 2.10, we infer that the $B V$-energy of $\left\langle T, d_{x_{0}}, r\right\rangle$ is given by

$$
\begin{aligned}
\mathcal{E}_{1,1}\left(\left\langle T, d_{x_{0}}, r\right\rangle, \partial B_{r}\left(x_{0}\right) \times \mathcal{Y}\right)= & \int_{\partial B_{r}\left(x_{0}\right)}\left|\nabla_{\tau} u_{\left(r, x_{0}\right)}\right| d \mathcal{H}^{n-1}+\left|D_{\tau}^{C} u\right|\left(\partial B_{r}\left(x_{0}\right)\right) \\
& +\int_{J_{c}(T) \cap \partial B_{r}\left(x_{0}\right)} \mathcal{L}_{T}(x) d \mathcal{H}^{n-2}(x),
\end{aligned}
$$

where $D_{\tau}$ and $\nabla_{\tau}$ denote the distributional derivative and the approximate gradient with respect to an orthonormal frame $\tau$ tangential to $\partial B_{r}\left(x_{0}\right)$, respectively.

Proof of Theorem 2.14. We make use of an inductive argument on the dimension $n$. More precisely, we will assume that Theorem 2.13 holds true in dimension $n-1$, and we use Theorem 1.7 in the case $n=2$. Therefore, taking into account the slicing properties previously outlined, we may and will assume that for every $x_{0} \in B^{n}$ and 
for a.e. radius $r \in\left(0, r\left(x_{0}\right)\right)$, where $r\left(x_{0}\right)>0$ is suitably chosen, by the inductive hypothesis we find a sequence of smooth functions $\left\{v_{k}\right\} \subset C^{1}\left(\partial B_{r}\left(x_{0}\right), \mathcal{Y}\right)$ such that

$$
G_{v_{k}} \rightarrow\left\langle T, d_{x_{0}}, r\right\rangle \quad \text { weakly in } \quad \mathcal{Z}_{n-1,1}\left(\partial B_{r}\left(x_{0}\right) \times \mathcal{Y}\right)
$$

and

$$
\int_{\partial B_{r}\left(x_{0}\right)}\left|D_{\tau} v_{k}\right| d \mathcal{H}^{n-1} \rightarrow \mathcal{E}_{1,1}\left(\left\langle T, d_{x_{0}}, r\right\rangle, \partial B_{r}\left(x_{0}\right) \times \mathcal{Y}\right) .
$$

In particular, we have that $v_{k} \rightarrow u_{\left(r, x_{0}\right)}$ weakly in the $B V$-sense. We divide the proof of Theorem 2.14 in six steps.

Step 1: Definition of the fine cover $\mathcal{F}_{m}$. We define for every $m \in \mathbb{N}$ a suitable fine cover $\mathcal{F}_{m}$ of $B^{n} \backslash J_{c}(T)$ consisting of closed balls of radius smaller than $1 / m$. To this aim, let $\mu_{d}$ and $\mu_{J c}$ be the mutually singular Radon measures on $B^{n}$ given for every Borel set $B \subset B^{n}$ by

$$
\begin{aligned}
\mu_{d}(B) & :=\int_{B}\left|\nabla u_{T}(x)\right| d x+\left|D^{C} u_{T}\right|(B), \\
\mu_{J c}(B) & :=\int_{J_{c}(T) \cap B} \mathcal{L}_{T}(x) d \mathcal{H}^{n-1}(x) .
\end{aligned}
$$

Definition 2.10 yields that the $B V$-energy of $T$ decomposes into the "diffuse" and "jump-concentration" part, i.e., setting

$$
\mu_{T}:=\mu_{d}+\mu_{J c},
$$

for every Borel set $B \subset B^{n}$ we have

$$
\mathcal{E}_{1,1}(T, B \times \mathcal{Y})=\mu_{T}(B)=\mu_{d}(B)+\mu_{J c}(B) .
$$

By the decomposition of the derivative $D u_{T}$, compare [2, Proposition 3.92], we infer that for any point $x_{0}$ in $B^{n} \backslash J_{c}(T)$ we have

$$
\liminf _{r \rightarrow 0} \frac{\mu_{T}\left(B_{r}\left(x_{0}\right)\right)}{r^{n-1}}=\liminf _{r \rightarrow 0} \frac{|D u|\left(B_{r}\left(x_{0}\right)\right)}{r^{n-1}}=0 .
$$

Moreover, since $\mu_{J c}=\mu_{J c}\left\llcorner J_{c}(T)\right.$, where $J_{c}(T)$ is a countably $\mathcal{H}^{n-1}$-rectifiable set, and $\mu_{T}\left(J_{c}(T)\right)<\infty$, for every $m \in \mathbb{N}$ we find a closed subset $J_{m} \subset J_{c}(T)$ such that

$$
J_{m} \subset J_{m+1} \quad \text { and } \quad \mu_{T}\left(J_{c}(T) \backslash J_{m}\right)=\mu_{J_{c}}\left(J_{c}(T) \backslash J_{m}\right)<\frac{1}{m} \quad \forall m .
$$

This yields in particular that

$$
\left|D^{J} u_{T}\right|\left(J_{u_{T}} \backslash J_{m}\right)<\frac{1}{m} .
$$


Setting now

$$
\Omega:=B^{n} \backslash J_{c}(T),
$$

$J_{m}$ being closed, for every $x_{0} \in \Omega$ there exists a positive radius $r=r\left(x_{0}, m\right)$, smaller than the distance of $x_{0}$ to the boundary $\partial B^{n}$, such that for every $0<r<$ $r\left(x_{0}, m\right)$

$$
\bar{B}_{r}\left(x_{0}\right) \cap J_{m}=\emptyset \text {. }
$$

Finally, by (4.1), if $x_{0} \in \Omega$, for every $0<r<r\left(x_{0}, m\right)$ we find a good radius $\rho \in(r / 2, r)$ such that

$$
\mathcal{E}_{1,1}\left(\left\langle T, d_{x_{0}}, \rho\right\rangle, \partial B_{\rho}\left(x_{0}\right) \times \mathcal{Y}\right) \leq \frac{2}{r} \mathcal{E}_{1,1}\left(T, \bar{B}_{r}\left(x_{0}\right) \times \mathcal{Y}\right) .
$$

We then denote by $\mathcal{F}_{m}$ the union of all the closed balls centered at points $x_{0} \in \Omega$ and with good radii $0<r<\min \left\{r\left(x_{0}, m\right) / 2,1 / m\right\}$ such that

$$
\mathcal{E}_{1,1}\left(\left\langle T, d_{x_{0}}, r\right\rangle, \partial B_{r}\left(x_{0}\right) \times \mathcal{Y}\right) \leq \frac{2}{r} \mathcal{E}_{1,1}\left(T, \bar{B}_{2 r}\left(x_{0}\right) \times \mathcal{Y}\right)
$$

and

$$
\frac{1}{(2 r)^{n-1}} \mathcal{E}_{1,1}\left(T, \bar{B}_{2 r}\left(x_{0}\right) \times \mathcal{Y}\right) \leq \frac{1}{m} .
$$

The above construction yields that $\mathcal{F}_{m}$ is a fine cover of $\Omega$ such that

$$
\bigcup \mathcal{F}_{m} \subset B^{n} \backslash J_{m}
$$

Step 2: Covering argument. We apply the following extension of the classical Vitali-Besicovitch covering theorem, see e.g. [2, Theorem 2.19], with respect to the positive Radon measure

$$
\mu:=\mathcal{L}^{n}+\mu_{T}=\mathcal{L}^{n}+\mu_{d}+\mu_{J c},
$$

where $\mathcal{L}^{n}$ is the Lebesgue measure and $\mu_{d}, \mu_{J_{c}}$ are given by (4.3). In the sequel, for any closed ball $B$ we will denote by $\widetilde{B}$ the closed ball centered as $B$ and with radius twice the radius of $B$, i.e.,

$$
\widetilde{B}:=\bar{B}_{2 r}\left(x_{0}\right) \quad \text { if } \quad B=\bar{B}_{r}\left(x_{0}\right) .
$$

Theorem 4.1 (Vitali-Besicovitch). Let $\Omega \subset \mathbb{R}^{n}$ be a bounded Borel set, and let $\mathcal{F}$ be a fine cover of $\Omega$ made of closed balls. For every positive Radon measure $\mu$ in $\mathbb{R}^{n}$ there is a disjoint countable family $\mathcal{F}^{\prime}$ of $\mathcal{F}$ such that

$$
\mu\left(\Omega \backslash \bigcup \mathcal{F}^{\prime}\right)=0
$$

Moreover, we have

$$
\sum_{B \in \mathcal{F}^{\prime}} \mu(\widetilde{B}) \leq C \cdot \mu(\Omega),
$$

where $C=C(n)>0$ is an absolute constant, only depending on the dimension $n$. 
Proof. Following the notation in [2, Theorem 2.19], setting $A_{0}:=\Omega$, for every $h \in$ $\mathbb{N}^{+}$, at the $h^{\text {th }}$ step we may and do apply the Besicovitch theorem [2, Theorem 2.17] by selecting the fine cover of $A_{h-1}$ given by all the closed balls $B$ of $\mathcal{F}$ such that the corresponding balls $\widetilde{B}$ are contained in $A_{h-1}$. Besicovitch's theorem yields the existence of a countable family made of closed balls $B$ which do not intersect more than $\xi$ times and such that their doubles $\widetilde{B}$ do not intersect more that $\eta$ times, where $\xi=\xi(n)$ and $\eta=\eta(n)$ are absolute constants. Therefore, the disjoint family $\mathcal{G}_{h}$ satisfies

$$
\sum_{B \in \mathcal{G}_{h}} \mu(\widetilde{B}) \leq \eta \cdot \mu\left(A_{h-1}\right)
$$

whereas, letting $A_{h}:=A_{h-1} \backslash \bigcup \mathcal{G}_{h}$, we have

$$
\mu\left(A_{h}\right) \leq \delta \mu\left(A_{h-1}\right), \quad \delta:=1-\frac{1}{2 \xi}<1 .
$$

Therefore, since $\mu\left(A_{h}\right) \leq \delta^{h} \cdot \mu\left(A_{0}\right)$ for every $h$, we obtain

$$
\sum_{B \in \mathcal{G}_{h}} \mu(\widetilde{B}) \leq \eta \cdot \delta^{h-1} \cdot \mu(\Omega)
$$

and finally

$$
\sum_{B \in \mathcal{F}^{\prime}} \mu(\widetilde{B})=\sum_{h=1}^{\infty} \sum_{B \in \mathcal{G}_{h}} \mu(\widetilde{B}) \leq \sum_{h=1}^{\infty} \eta \cdot \delta^{h-1} \cdot \mu(\Omega)
$$

which yields the assertion, by taking $C:=\eta /(1-\delta)$.

By Theorem 4.1 we obtain for every $m$ a suitable denumerable disjoint family $\mathcal{F}_{m}^{\prime}$ of closed balls contained in $B^{n} \backslash J_{m}$ and with radii smaller than $1 / m$. We finally label

$$
\mathcal{F}_{m}^{\prime}=\left\{B_{j}\right\}_{j=1}^{\infty}, \quad \Omega_{m}:=\bigcup_{j=1}^{\infty} B_{j}
$$

and notice that

$$
\mu_{J c}\left(\Omega_{m}\right) \leq \mu_{J c}\left(B^{n} \backslash J_{m}\right)<\frac{1}{m} \quad \text { and } \quad \mu_{d}\left(B^{n} \backslash \Omega_{m}\right)=0 .
$$

Step 3: Smoothing of the boundary data. If $B_{j}=\bar{B}_{r}\left(x_{0}\right) \in \mathcal{F}_{m}^{\prime}$, arguing as in Gagliardo's theorem [11, Theorem 1.II], that states the existence of a $W^{1,1}$ extension of any $L^{1}$-function, we are able to modify the boundary datum $\left\langle T, d_{x_{0}}, r\right\rangle$ to a smooth $W^{1,1}$-map with values into $\mathcal{Y}$. This can be done by paying an arbitrary small amount of energy. 
More precisely, due to the inductive hypothesis, see (4.2), we find a sequence of smooth maps $\left\{v_{h}^{(j)}\right\} \subset W^{1,1}\left(\partial B_{j}, \mathcal{Y}\right)$ such that $\left\|v_{h}^{(j)}-u_{\mid \partial B_{j}}\right\|_{L^{1}\left(\partial B_{j}\right)} \rightarrow 0$,

$$
G_{v_{h}^{(j)}} \rightarrow\left\langle T, d_{x_{0}}, r\right\rangle \quad \text { weakly in } \quad \mathcal{Z}_{n-1,1}\left(\partial B_{j} \times \mathcal{Y}\right)
$$

as $h \rightarrow \infty$ and

$$
\int_{\partial B_{j}}\left|D_{\tau} v_{h}^{(j)}\right| d \mathcal{H}^{n-1} \leq \mathcal{E}_{1,1}\left(\left\langle T, d_{x_{0}}, r\right\rangle, \partial B_{j} \times \mathcal{Y}\right) \cdot\left(1+2^{-h}\right)
$$

for every $h$. Taking $k$ sufficiently large, we now define a map $W_{k}^{(j)} \in W^{1,1}\left(A_{\rho_{k}}^{r}, \mathbb{R}^{N}\right)$, where $0<\rho_{k}<r$ and $A_{\rho}^{r}$ denotes the annulus

$$
A_{\rho}^{r}:=\bar{B}_{r}\left(x_{0}\right) \backslash B_{\rho}\left(x_{0}\right), \quad 0<\rho<r,
$$

in such a way that $W_{k \mid \partial B_{r}\left(x_{0}\right)}^{(j)}=u_{\mid \partial B_{r}\left(x_{0}\right)}$ in the sense of traces,

$$
W_{k}^{(j)}\left(x_{0}+\rho_{k} \frac{x-x_{0}}{\left|x-x_{0}\right|}\right)=v_{k}^{(j)}\left(x_{0}+r \frac{x-x_{0}}{\left|x-x_{0}\right|}\right)
$$

and the energy $\int_{A_{\rho_{k}}^{r}}\left|D W_{k}^{(j)}\right| d x$ is arbitrarily small, if $\rho_{k} \nearrow r$ sufficiently rapidly.

The function $W_{k}^{(j)}$ is obtained by parametrizing in a sequence of annuli of the type $A_{\rho_{h}}^{\rho_{h+1}}$, for a suitable sequence $\left\{\rho_{h}\right\}_{h \geq k}$ of radii $\rho_{h} \nearrow r$, the affine homotopies

$$
t_{h} v_{h}^{(j)}+\left(1-t_{h}\right) v_{h+1}^{(j)}, \quad t_{h}=t_{h}(\rho) \in[0,1], \quad \rho:=\left|x-x_{0}\right|,
$$

where $t_{h}(\rho)$ is the affine map such that $t_{h}\left(\rho_{h}\right)=1$ and $t_{h}\left(\rho_{h+1}\right)=0$. Therefore, if we show that for every $t \in[0,1]$ and $h \geq k$ the $L^{\infty}$-distance of $t v_{h}^{(j)}+(1-t) v_{h+1}^{(j)}$ from $\mathcal{Y}$ is small, we find that

$$
\operatorname{dist}\left(W_{k}^{(j)}(x), \mathcal{Y}\right)<\varepsilon_{0} \quad \text { for } \mathcal{L}^{n} \text {-a.e. } x \in A_{\rho_{k}}^{r}
$$

and hence we may and do define $w_{k}^{(j)}:=\Pi_{\varepsilon_{0}} \circ W_{k}^{(j)}$ on $A_{\rho_{k}}^{r}$, where $\Pi_{\varepsilon_{0}}$ is the Lipschitz projection on $\mathcal{Y}$ given by Remark 1.9.

To prove (4.9), due to the $L^{1}$-convergence and to (4.8), by applying Poincaré inequality we find an absolute constant $c_{n}>0$ such that, if $k$ is sufficiently large, for $\mathcal{H}^{n-1}$-a.e. $x \in \partial B_{r}\left(x_{0}\right)$ and every $h \geq k$ we have

$$
\begin{aligned}
\int_{\partial B_{r}\left(x_{0}\right)}\left|v_{h}^{(j)}(x)-u(y)\right| d \mathcal{H}^{n-1}(y) \\
\quad \leq \int_{\partial B_{r}\left(x_{0}\right)}\left|v_{h}^{(j)}(x)-v_{h}^{(j)}(y)\right| d \mathcal{H}^{n-1}(y)+\left\|v_{h}^{(j)}-u\right\|_{L^{1}\left(\partial B_{r}\left(x_{0}\right)\right)} \\
\leq c_{n} r \int_{\partial B_{r}\left(x_{0}\right)}\left|D_{\tau} v_{h}^{(j)}\right| d \mathcal{H}^{n-1}+\left\|v_{h}^{(j)}-u\right\|_{L^{1}\left(\partial B_{r}\left(x_{0}\right)\right)} \\
\leq 2 c_{n} r \cdot \mathcal{E}_{1,1}\left(\left\langle T, d_{x_{0}}, r\right\rangle, \partial B_{j} \times \mathcal{Y}\right) .
\end{aligned}
$$


As a consequence, by (4.4) and (4.5) we obtain

$$
\int_{\partial B_{r}\left(x_{0}\right)}\left|v_{h}^{(j)}(x)-u(y)\right| d \mathcal{H}^{n-1}(y) \leq 2^{n+1} \cdot c_{n} \cdot \frac{r^{n-1}}{m}
$$

and hence, by convexity, for any $t \in[0,1]$ we have

$$
\begin{array}{r}
\int_{\partial B_{r}\left(x_{0}\right)}\left|t v_{h}^{(j)}(x)+(1-t) v_{h+1}^{(j)}(x)-u(y)\right| d \mathcal{H}^{n-1}(y) \\
\leq \int_{\partial B_{r}\left(x_{0}\right)}\left|v_{h}^{(j)}(x)-u(y)\right| d \mathcal{H}^{n-1}(y)+\int_{\partial B_{r}\left(x_{0}\right)}\left|v_{h+1}^{(j)}(x)-u(y)\right| d \mathcal{H}^{n-1}(y) \\
<\mathcal{H}^{n-1}\left(\partial B_{r}\left(x_{0}\right)\right) \cdot \varepsilon_{0}
\end{array}
$$

provided that $m \in \mathbb{N}$ is large enough so that $2^{n+2} \cdot c_{n} \cdot 1 / m<\varepsilon_{0} \cdot n \cdot \omega_{n}$, where $\omega_{n}$ is the measure of the unit $n$-ball. Therefore, arguing as in Schoen-Uhlenbeck density theorem [21], we obtain

$$
\operatorname{dist}\left(t v_{h}^{(j)}(x)+(1-t) v_{h+1}^{(j)}(x), \mathcal{Y}\right)<\varepsilon_{0} \quad \text { for } \mathcal{H}^{n-1} \text {-a.e. } x \in \partial B_{r}\left(x_{0}\right),
$$

which yields (4.9), as required.

We remark that due to the strong convergence (4.7) (4.8), the sequence $\left\{w_{k}^{(j)}\right\}_{k}$ this way obtained also satisfies the boundary condition

$$
\left\langle G_{w_{k}^{(j)}}, d_{x_{0}}, r\right\rangle=\left\langle T, d_{x_{0}}, r\right\rangle .
$$

Finally, for future use, we extend $w_{k}^{(j)}$ to the whole ball $B_{j}$ by the map $\widetilde{w}_{k}^{(j)}$ : $\bar{B}_{\rho_{k}}\left(x_{0}\right) \rightarrow \mathcal{Y}$ given by

$$
\widetilde{w}_{k}^{(j)}(x):=\left\{\begin{array}{lll}
w_{k}^{(j)} \circ \psi_{(r, \sigma)}(x) & \text { if } & x \in A_{r-2 \sigma}^{r-\sigma} \\
u \circ \phi_{(r, \sigma)}(x) & \text { if } & x \in B_{r-2 \sigma}\left(x_{0}\right),
\end{array}\right.
$$

where $\sigma:=r-\rho_{k}, \psi_{(r, \sigma)}: A_{r-2 \sigma}^{r-\sigma} \rightarrow A_{r-\sigma}^{r}$ is the reflection map

$$
\psi_{(r, \sigma)}(x):=\left(-\left|x-x_{0}\right|+2(r-\sigma)\right) \frac{x-x_{0}}{\left|x-x_{0}\right|}
$$

and $\phi_{(r, \sigma)}: B_{r-2 \sigma}\left(x_{0}\right) \rightarrow B_{r}\left(x_{0}\right)$ is the homothetic map

$$
\phi_{(r, \sigma)}(x):=x_{0}+\frac{r}{r-2 \sigma}\left(x-x_{0}\right) .
$$

Notice that $\widetilde{w}_{k}^{(j)}$ is smooth on $A_{r-2 \sigma}^{r-\sigma}$ and that, taking $\sigma$ small, by the property above we may and do assume that

$$
\left|D \widetilde{w}_{k}^{(j)}\right|\left(\bar{B}_{\rho_{k}}\left(x_{0}\right)\right) \leq 2|D u|\left(\bar{B}_{r}\left(x_{0}\right)\right) .
$$


Step 4: Approximation on the balls of $\mathcal{F}_{m}^{\prime}$. Let $B_{j}=\bar{B}_{r}\left(x_{0}\right) \in \mathcal{F}_{m}^{\prime}$. Making use of arguments from [5], we now define an approximating sequence on $B_{j}$.

We first fix some notation. For any $\rho>0$, we let

$$
Q_{\rho}^{n}:=[-\rho, \rho]^{n} \subset \mathbb{R}^{n}
$$

denote the $n$-dimensional cube of side $2 \rho$ and $\Sigma_{\rho}^{i}$ the $i$-dimensional skeleton of $Q_{\rho}^{n}$, so that $\bigcup \Sigma_{\rho}^{n-1}=\partial Q_{\rho}^{n}$. Let $\|x\|:=\max \left\{\left|x_{1}\right|, \ldots,\left|x_{n}\right|\right\}$, so that

$$
Q_{\rho}^{n}=\left\{x \in \mathbb{R}^{n}:\|x\| \leq \rho\right\}, \quad \partial Q_{\rho}^{n}=\left\{x \in \mathbb{R}^{n}:\|x\|=\rho\right\} .
$$

If $v: Q_{\rho}^{n} \rightarrow \mathbb{R}^{N}$ is any given $B V$-function, and $F$ is any $i$-face of $\Sigma_{\rho}^{i}$, in the sequel we will denote

$$
E_{1,1}(v, F):=\left|D v_{\mid F}\right|(F)
$$

where $D v_{\mid F}$ is the distributional derivative of the restriction $v_{\mid F}$ of $v$ to $F$, and we let

$$
E_{1,1}\left(v, \Sigma_{\rho}^{i}\right):=\sum_{F \in \Sigma_{\rho}^{i}} E_{1,1}(v, F) .
$$

Recall that $\mathcal{Y} \subset \mathbb{R}^{N}$, and denote by

$$
B \mathcal{Y}(y, \varepsilon):=\bar{B}^{N}(y, \varepsilon) \cap \mathcal{Y}
$$

the intersection of $\mathcal{Y}$ with the closed $N$-ball of radius $\varepsilon$ centered at $y$. If $y \in \mathcal{Y}$ and $0<\varepsilon<\varepsilon_{0}$, we let $\Psi_{(y, \varepsilon)}: \mathbb{R}^{N} \rightarrow \bar{B}_{\mathcal{Y}}(y, \varepsilon)$ be the retraction map given by $\Psi_{(y, \varepsilon)}(z):=\Pi_{\varepsilon} \circ \xi_{(y, \varepsilon)}$, where

$$
\xi_{(y, \varepsilon)}(z):=\left\{\begin{array}{llll}
z & \text { if } & z \in \bar{B}^{N}(y, \varepsilon) \\
\varepsilon \frac{z-y}{|z-y|} & \text { if } & z \in \mathbb{R}^{N} \backslash \bar{B}^{N}(y, \varepsilon)
\end{array}\right.
$$

and $\Pi_{\varepsilon}: \mathcal{Y}_{\varepsilon} \rightarrow \mathcal{Y}$ is the projection map given by Remark 1.9. Of course, $\Psi_{(y, \varepsilon)}$ is a Lipschitz continuous function with $\operatorname{Lip} \Psi_{(y, \varepsilon)}=\operatorname{Lip} \Pi_{\varepsilon} \rightarrow 1^{+}$as $\varepsilon \rightarrow 0^{+}$.

First, letting $\rho=\rho_{k}$ from Step 3, by means of a deformation and slicing argument, we may and do define a bilipschitz homeomorphism $\psi_{j}: \bar{B}_{\rho}\left(x_{0}\right) \rightarrow Q_{\rho}^{n}$ such that $\left\|D \psi_{j}\right\|_{\infty} \leq K,\left\|D \psi_{j}^{-1}\right\|_{\infty} \leq K$ for some absolute constant $K>0$, only depending on $n$. Moreover, we may and do define $\psi_{j}$ in such a way that

$$
\psi_{j}\left(\bar{B}_{R}\left(x_{0}\right)\right)=Q_{R}^{n} \quad \forall R \in(\rho / 2, \rho) .
$$

Finally, for any given $B V$-function $\widetilde{v}: \bar{B}_{\rho}\left(x_{0}\right) \rightarrow \mathcal{Y}$, smooth on $\partial B_{\rho}\left(x_{0}\right)$, if $v_{j}: Q_{\rho}^{n} \rightarrow \mathcal{Y}$ is the corresponding map given by $v_{j}:=\widetilde{v} \circ \psi_{j}^{-1}$, we also may and do define $\psi_{j}$ in such a way that

$$
E_{1,1}\left(v_{j}, \Sigma_{\rho}^{i}\right) \leq C \cdot \frac{1}{\rho} \cdot E_{1,1}\left(v_{j}, \Sigma_{\rho}^{i+1}\right) \quad \forall i=1, \ldots, n-2,
$$

where $C>0$ is an absolute constant, not depending on $\tilde{v}$. 
Taking $\widetilde{v}=\widetilde{v}_{j}:=\widetilde{w}_{k}^{(j)}$ from (4.12), i.e., letting

$$
v_{j}:=\widetilde{w}_{k}^{(j)} \circ \psi_{j}^{-1}: Q_{\rho}^{n} \rightarrow \mathcal{Y},
$$

by (4.8) and (4.15) we readily infer that

$$
E_{1,1}\left(v_{j}, \Sigma_{\rho}^{i}\right) \leq 2 C K \rho^{i-n+1} \mathcal{E}_{1,1}\left(\left\langle T, d_{x_{0}}, r\right\rangle, \partial B_{j} \times \mathcal{Y}\right) \quad \forall i=1, \ldots, n-1
$$

and hence, by (4.4), that

$$
E_{1,1}\left(v_{j}, \Sigma_{\rho}^{i}\right) \leq \widetilde{C} \rho^{i-n} \mathcal{E}_{1,1}\left(T, \bar{B}_{2 r}\left(x_{0}\right) \times \mathcal{Y}\right) \quad \forall i=1, \ldots, n-1 .
$$

On the other hand, since we may assume $\rho>r / 2$, due to (4.5) and (4.13), by (4.17) we also obtain

$$
\frac{1}{\rho^{i-1}} E_{1,1}\left(v_{j}, \Sigma_{\rho}^{i}\right) \leq \widetilde{C} \frac{1}{m} \quad \forall i=1, \ldots, n,
$$

where in the above formulas $\widetilde{C}>0$ is an absolute constant.

Remark 4.2. Let $\varepsilon_{m}:=1 / \sqrt{m}$. By the Sobolev embedding theorem, if $m \in \mathbb{N}$ is sufficiently large, e.g., $m \geq 4 \widetilde{C}^{2}$, the inequality (4.18), with $i=1$, yields that the oscillation of $v_{j}$ on the 1 -skeleton $\Sigma_{\rho}^{1}$ is smaller than $\varepsilon_{m} / 2$, if $v_{j}$ is smooth. Therefore, the image $v_{j}\left(\Sigma_{\rho}^{1}\right)$ is contained in a small geodesic ball $B_{\mathcal{Y}}\left(y_{j}, \varepsilon_{m} / 2\right)$ centered at some given point $y_{j} \in \mathcal{Y}$. Actually, since the total variation of 1dimensional $B V$-functions estimates the oscillation, we infer that the above property holds for $B V$-function $v_{j}$, provided that in (4.18) we consider the total variation of the 1-dimensional restriction of $v$ to $\Sigma_{\rho}^{1}$. We also notice that

$$
\lim _{m \rightarrow+\infty} \varepsilon_{m} \cdot m=+\infty
$$

whereas, on account of Remark 1.9,

$$
\operatorname{Lip} \Psi_{\left(y_{j}, \varepsilon_{m}\right)}=\operatorname{Lip} \Pi_{\varepsilon_{m}} \rightarrow 1^{+} \quad \text { as } \quad m \rightarrow+\infty .
$$

The case $n=2$. In case of dimension $n=2$, we define $w_{j}: Q_{\rho}^{2} \rightarrow B \mathcal{Y}\left(y_{j}, \varepsilon_{m}\right)$ by

$$
w_{j}:=\Psi_{\left(y_{j}, \varepsilon_{m}\right)} \circ v_{j},
$$

where $v_{j}$ is given by (4.16), so that

$$
\left|D w_{j}\right|\left(Q_{\rho}^{2}\right)=: E_{1,1}\left(w_{j}, Q_{\rho}^{2}\right) \leq\left(\operatorname{Lip} \Pi_{\varepsilon_{m}}\right) \cdot E_{1,1}\left(v_{j}, Q_{\rho}^{2}\right) .
$$

Remark 4.2 yields that $w_{j}$ agrees with $v_{j}$ on the boundary of $Q_{\rho}^{2}$. Moreover, letting $R:=\rho-\sigma$, by (4.12), (4.14) and (4.16) we infer that $w_{j}$ is smooth on $Q_{\rho}^{2} \backslash Q_{R}^{2}$ and that

$$
w_{j}(x)=\Psi_{\left(y_{j}, \varepsilon_{m}\right)} \circ\left(u \circ \phi_{(r, \sigma)}\right) \circ \psi_{j}^{-1}(x) \quad \forall x \in Q_{R}^{2} .
$$


Since the image of $Q_{R}^{2}$ by $w_{j}$ is contained in the geodesic ball $B \mathcal{Y}\left(y_{j}, \varepsilon_{m}\right)$, by means of a convolution argument we can approximate $w_{j}$ on $Q_{R}^{2}$ by a smooth sequence $v_{\varepsilon}^{(j)}: Q_{R}^{2} \rightarrow \bar{B}^{N}\left(y_{j}, \varepsilon_{m}\right)$ which converges in the $L^{1}$-sense to $w_{j \mid Q_{R}^{2}}$ and with total variation converging to the total variation $\left|D w_{j}\right|\left(Q_{R}^{2}\right)$. We finally set $w_{\varepsilon}^{(j)}:=\Pi_{\varepsilon_{m}} \circ v_{\varepsilon}^{(j)}: Q_{R}^{2} \rightarrow \mathcal{Y}$, see Remark 1.9, so that clearly $w_{\varepsilon}^{(j)} \rightarrow w_{j}$ weakly in $B V\left(Q_{R}^{2}, \mathbb{R}^{N}\right)$, whereas

$$
E_{1,1}\left(w_{\varepsilon}^{(j)}, Q_{R}^{2}\right) \leq\left(\operatorname{Lip} \Pi_{\varepsilon_{m}}\right) \cdot E_{1,1}\left(v_{\varepsilon}^{(j)}, Q_{R}^{2}\right),
$$

so that

$$
\limsup _{\varepsilon \rightarrow 0} E_{1,1}\left(w_{\varepsilon}^{(j)}, Q_{R}^{2}\right) \leq\left(\operatorname{Lip} \Pi_{\varepsilon_{m}}\right)^{2} \cdot E_{1,1}\left(v_{j}, Q_{R}^{2}\right) .
$$

Moreover, by suitably defining the convolution kernel, we may and do assume that the traces are equal, so that $w_{\varepsilon \mid \partial Q_{R}^{2}}^{(j)}=v_{\varepsilon \mid \partial Q_{R}^{2}}^{(j)}=w_{j \mid \partial Q_{R}^{2}}$. Most importantly, by the construction we may and do assume that the boundaries of the graphs agree on $\partial Q_{R}^{2}$, so that

$$
\partial G_{w_{\varepsilon}^{(j)}}\left\llcorner\partial Q_{R}^{2} \times \mathcal{Y}=\partial G_{v_{\varepsilon}^{(j)}}\left\llcorner\partial Q_{R}^{2} \times \mathcal{Y}=\partial G_{w_{j}}\left\llcorner\partial Q_{R}^{2} \times \mathcal{Y} .\right.\right.\right.
$$

Finally, letting $w_{\varepsilon}^{(j)}=w_{j}$ on $Q_{\rho}^{2} \backslash Q_{R}^{2}$, we define $u_{k}^{(j)}: \bar{B}_{r}\left(x_{0}\right) \rightarrow \mathcal{Y}$ by

$$
u_{k}^{(j)}(x):=\left\{\begin{array}{lll}
w_{\varepsilon_{k}}^{(j)} \circ \psi_{j}(x) & \text { if } & x \in \bar{B}_{\rho}\left(x_{0}\right) \\
w_{k}^{(j)}(x) & \text { if } & x \in \bar{B}_{r}\left(x_{0}\right) \backslash \bar{B}_{\rho}\left(x_{0}\right),
\end{array}\right.
$$

where $\rho=\rho_{k}$ and $\varepsilon_{k} \searrow 0$ along a sequence.

The case $n \geq 3$. For $\delta:=\rho(1-\eta)$, where $\eta:=1 / q$ and $q \in \mathbb{N}^{+}$, we let $\Phi_{(\rho, \delta)}: Q_{\rho}^{n} \rightarrow Q_{\delta}^{n}$ be given by

$$
\Phi_{(\rho, \delta)}(x):=(1-\eta) x .
$$

Note that

$$
E_{1,1}\left(v_{j} \circ \Phi_{(\rho, \delta)}^{-1}, \Sigma_{\delta}^{i}\right)=(1-\eta)^{i-1} E_{1,1}\left(v_{j}, \Sigma_{\rho}^{i}\right),
$$

so that (4.18) yields

$$
\frac{1}{\delta^{i-1}} E_{1,1}\left(v_{j} \circ \Phi_{(\rho, \delta)}^{-1}, \Sigma_{\delta}^{i}\right) \leq \widetilde{C} \frac{1}{m} \quad \forall i=1, \ldots, n .
$$

Define $w_{j}: Q_{\delta}^{n} \rightarrow B \mathcal{Y}\left(y_{j}, \varepsilon_{m}\right)$ by

$$
w_{j}:=\Psi_{\left(y_{j}, \varepsilon_{m}\right)} \circ v_{j} \circ \Phi_{(\rho, \delta)}^{-1},
$$


where $v_{j}$ is given by (4.16), so that

$$
\left|D w_{j}\right|\left(Q_{\delta}^{n}\right)=: E_{1,1}\left(w_{j}, Q_{\delta}^{n}\right) \leq\left(\operatorname{Lip} \Pi_{\varepsilon_{m}}\right) \cdot E_{1,1}\left(v_{j} \circ \Phi_{(\rho, \delta)}^{-1}, Q_{\delta}^{n}\right) .
$$

Remark 4.2 yields that $w_{j}$ agrees with $v_{j} \circ \Phi_{(\rho, \delta)}^{-1}$ on the 1-skeleton $\Sigma_{\delta}^{1}$ of $Q_{\delta}^{n}$. Moreover, letting $R:=(\rho-\sigma)(1-\eta)$, by (4.12) and (4.14) we infer that $w_{j}$ is smooth on $Q_{\delta}^{n} \backslash Q_{R}^{n}$ and that

$$
w_{j}(x)=\Psi_{\left(y_{j}, \varepsilon_{m}\right)} \circ\left(u \circ \phi_{(r, \sigma)}\right) \circ \psi_{j}^{-1} \circ \Phi_{(\rho, \delta)}^{-1}(x) \quad \forall x \in Q_{R}^{n} .
$$

Now, since the image of $Q_{R}^{n}$ by $w_{j}$ is contained in the geodesic ball $B \mathcal{Y}\left(y_{j}, \varepsilon_{m}\right)$, as in the case of dimension $n=2$, we approximate $w_{j}$ by a smooth sequence $v_{\varepsilon}^{(j)}: Q_{R}^{n} \rightarrow \bar{B}^{N}\left(y_{j}, \varepsilon_{m}\right)$ which converges in the $L^{1}$-sense to $w_{j \mid Q_{R}^{n}}$, with total variation converging to the total variation $\left|D w_{j}\right|\left(Q_{R}^{n}\right)$. Setting $w_{\varepsilon}^{(j)}:=\Pi_{\varepsilon_{m}} \circ v_{\varepsilon}^{(j)}$ : $Q_{R}^{n} \rightarrow \mathcal{Y}$, we have $w_{\varepsilon}^{(j)} \rightarrow w_{j}$ weakly in $B V\left(Q_{R}^{n}, \mathbb{R}^{N}\right)$, whereas

$$
E_{1,1}\left(w_{\varepsilon}^{(j)}, Q_{R}^{n}\right) \leq\left(\operatorname{Lip} \Pi_{\varepsilon_{m}}\right) \cdot E_{1,1}\left(v_{\varepsilon}^{(j)}, Q_{R}^{n}\right),
$$

so that again we have

$$
\limsup _{\varepsilon \rightarrow 0} E_{1,1}\left(w_{\varepsilon}^{(j)}, Q_{R}^{n}\right) \leq\left(\operatorname{Lip} \Pi_{\varepsilon_{m}}\right)^{2} \cdot E_{1,1}\left(v_{j} \circ \Phi_{(\rho, \delta)}^{-1}, Q_{R}^{n}\right) .
$$

Moreover, we may and do assume that the traces of $w_{\varepsilon}^{(j)}$ and $w_{j}$ on $\partial Q_{R}^{n}$ are equal, $w_{\varepsilon \mid \partial Q_{R}^{n}}^{(j)}=w_{j \mid \partial Q_{R}^{n}}$, and that the boundaries of the graphs agree on $\partial Q_{R}^{n}$, i.e.,

$$
\partial G_{w_{\varepsilon}^{(j)}}\left\llcorner\partial Q_{R}^{n} \times \mathcal{Y}=\partial G_{w_{j}}\left\llcorner\partial Q_{R}^{n} \times \mathcal{Y} .\right.\right.
$$

Finally set $w_{\varepsilon}^{(j)}=w_{j}$ on $Q_{\delta}^{n} \backslash Q_{R}^{n}$.

In order to extend the approximating map to $Q_{\rho}^{n} \backslash Q_{\delta}^{n}$, we use an argument from [5]. If $S_{h}$ is one of the $(n-1)$-faces of $\Sigma_{\rho}^{n-1}$, where $h=1, \ldots, 2 n$, we may and do define a partition of $S_{h}$ into $(q+1)^{n-1}$ small $(n-1)$-dimensional "cubes" $C_{l, h}$ in such a way that the following facts hold:

i) If $\left[C_{l, h}\right]_{i}$ denotes the $i$-dimensional skeleton of the boundary of $C_{l, h}$, the restriction of $v_{j}$ to $\left[C_{l, h}\right]_{i}$ belongs to $W^{1,1}$, for every $i=1, \ldots, n-2$; in particular, $v_{j}$ is continuous on the 1 -skeleton $\left[C_{l, h}\right]_{1}$.

ii) If $n=3$, we have

$$
\sum_{l=1}^{(q+1)^{2}} E_{1,1}\left(v_{j}, \partial C_{l, h}\right) \leq K\left(E_{1,1}\left(v_{j}, \partial S_{h}\right)+\frac{q}{\rho} E_{1,1}\left(v_{j}, S_{h}\right)\right),
$$

where $K>0$ is an absolute constant. 
iii) If $n \geq 4$, and $\left[S_{h}\right]_{i}$ denotes the $i$-dimensional skeleton of $S_{h}$, for every $i=$ $1, \ldots, n-2$ we have

$$
\sum_{l=1}^{(q+1)^{n-1}} E_{1,1}\left(v_{j},\left[C_{l, h}\right]_{i}\right) \leq K \cdot \sum_{t=i}^{n-1}\left(\frac{q}{\rho}\right)^{t-i} \cdot E_{1,1}\left(v_{j},\left[S_{h}\right]_{t}\right)
$$

where $K>0$ is an absolute constant.

iv) All the $C_{l, h}$ 's are bilipschitz homeomorphic to the $(n-1)$-cube $[-\rho / q, \rho / q]^{n-1}$ by linear maps $f_{l, h}$ such that $\left\|D f_{l, h}\right\|_{\infty} \leq K,\left\|D f_{l, h}^{-1}\right\|_{\infty} \leq K$.

Moreover, the inequality (4.18), with $i=2, \ldots, n-1$, yields that if $m \in \mathbb{N}$ is sufficiently large, and $q$ satisfies

$$
q<\frac{1}{5(n-2) \widetilde{C}} \cdot \frac{\varepsilon_{m}}{2} \cdot m,
$$

we may and do define the partition of $S_{h}$ in such a way that

$$
E_{1,1}\left(v_{j},\left[C_{l, h}\right]_{1}\right) \leq \frac{\varepsilon_{m}}{2} \quad \forall l=1, \ldots,(q+1)^{n-1}, \quad \forall h=1, \ldots, 2 n .
$$

Therefore, in the sequel we will take

$$
q:=\text { integer part of }\left(\widehat{C} \cdot \varepsilon_{m} \cdot m\right)
$$

for some fixed constant $\widehat{C}>0$, say $\widehat{C}:=1 /(12(n-2) \widetilde{C})$.

Remark 4.3. Again by Remark 4.2, since the image $v_{j}\left(\Sigma_{\rho}^{1}\right)$ is contained in $B \mathcal{Y}\left(y_{j}, \varepsilon_{m} / 2\right)$, the inequalities in (4.28) yield that the image of $\left[C_{l, h}\right]_{1}$ by $v_{j}$ is contained in the geodesic ball $B \mathcal{Y}\left(y_{j}, \varepsilon_{m}\right)$ for every $l$ and $h$. By (4.23), this yields that the function $w_{j}$, and hence the $w_{\varepsilon}^{(j)}$ 's, agrees with $v_{j} \circ \Phi_{(\rho, \delta)}^{-1}$ on the 1-skeleton $\widetilde{\Sigma}_{\delta}^{1}$ of $\partial Q_{\delta}^{n}$ given by

$$
\widetilde{\Sigma}_{\delta}^{1}:=\Phi_{(\rho, \delta)}\left(\bigcup_{h=1}^{2 n} \bigcup_{l=1}^{(q+1)^{n-1}}\left[C_{l, h}\right]_{1}\right) .
$$

Finally, if $\pi_{(\rho, \delta)}: Q_{\rho}^{n} \backslash Q_{\delta}^{n} \rightarrow \partial Q_{\rho}^{n}$ is the projection map $\pi_{(\rho, \delta)}(x):=\rho x /\|x\|$, setting

$$
\mathcal{M}_{(\rho, \delta)}:=\pi_{(\rho, \delta)}^{-1} \circ \Phi_{(\rho, \delta)}\left(\bigcup_{h=1}^{2 n} \bigcup_{l=1}^{(q+1)^{n-1}} \partial C_{l, h}\right)
$$

it turns out that the $(n-1)$-skeleton

$$
\mathcal{N}_{(\rho, \delta)}:=\mathcal{M}_{(\rho, \delta)} \cup \partial Q_{\rho}^{n} \cup \partial Q_{\delta}^{n}
$$


is the union of boundary of $n$-dimensional "cubes" $Q_{l, h}$, satisfying $C_{l, h} \subset \partial Q_{l, h}$ for every $l$ and $h$, that partition $Q_{\rho}^{n} \backslash Q_{\delta}^{n}$. Moreover, each $Q_{l, h}$ is bilipschitz homeomorphic to the $n$-cube $[-\rho / q, \rho / q]^{n}$ by linear maps $\widetilde{f}_{l, h}$ such that $\left\|D \widetilde{f}_{l, h}\right\|_{\infty} \leq$ $K,\left\|D \tilde{f}_{l, h}^{-1}\right\|_{\infty} \leq K$, where $K>0$ is an absolute constant.

We now extend the approximating map to the interior of $Q_{\rho}^{n} \backslash Q_{\delta}^{n}$, first considering the simpler case $n=3$.

The case $n=3$. We first set $w_{j}:=v_{j}$ on $\partial Q_{\rho}^{3}$ and

$$
w_{j}:=v_{j} \circ \pi_{(\rho, \delta)}(x) \quad \text { on } \mathcal{M}_{(\rho, \delta)} .
$$

By Remark 4.3, the function $w_{j}$ is smooth on the 2-skeleton $\mathcal{N}_{(\rho, \delta)}$. We then extend $w_{j}$ to the whole of $Q_{\rho}^{3} \backslash Q_{\delta}^{3}$ by means of a radial extension on each cube $Q_{l, h}$, i.e., by setting

$$
w_{j}(x):=w_{j}\left(\tilde{f}_{l, h}^{-1}\left(\frac{\rho}{q} \cdot \frac{\widetilde{f}_{l, h}(x)}{\left\|\widetilde{f}_{l, h}(x)\right\|}\right)\right), \quad x \in Q_{l, h}, \quad \forall l, h .
$$

The function $w_{j}$ this way constructed is smooth on the closure of $Q_{\rho}^{3} \backslash Q_{\delta}^{3}$, up to a discrete set of points. Moreover, denoting by $C>0$ an absolute constant, possibly varying from line to line, but not depending on $\rho$ or $m$, we have

$$
E_{1,1}\left(w_{j}, Q_{l, h}\right) \leq C \frac{\rho}{q} E_{1,1}\left(w_{j}, \partial Q_{l, h}\right)
$$

whereas

$$
E_{1,1}\left(w_{j}, \partial Q_{l, h}\right) \leq C\left(E_{1,1}\left(v_{j}, C_{l, h}\right)+\frac{\rho}{q} E_{1,1}\left(v_{j}, \partial C_{l, h}\right)\right) .
$$

Therefore, by (4.26), and by summing on $l$ and $h$, we estimate

$$
E_{1,1}\left(w_{j}, Q_{\rho}^{3} \backslash Q_{\delta}^{3}\right) \leq C\left(\frac{\rho}{q} E_{1,1}\left(v_{j}, \Sigma_{\rho}^{2}\right)+\left(\frac{\rho}{q}\right)^{2} E_{1,1}\left(v_{j}, \Sigma_{\rho}^{1}\right)\right) .
$$

Finally, by (4.29) and (4.17) we obtain, for $m>1 / \widehat{C}^{2}$,

$$
E_{1,1}\left(w_{j}, Q_{\rho}^{3} \backslash Q_{\delta}^{3}\right) \leq C \frac{1}{\varepsilon_{m} \cdot m} \mathcal{E}_{1,1}\left(T, \bar{B}_{2 r}\left(x_{0}\right) \times \mathcal{Y}\right) .
$$

The case $n \geq 4$. According to Remark 4.3, we first set $w_{j}:=v_{j}$ on $\partial Q_{\rho}^{n}$ and

$$
w_{j}:=v_{j} \circ \pi_{(\rho, \delta)}(x) \quad \text { on } \quad \pi_{(\rho, \delta)}^{-1}\left(\widetilde{\Sigma}_{\delta}^{1}\right) .
$$

To extend $w_{j}$ to the whole of $Q_{\rho}^{n} \backslash Q_{\delta}^{n}$, we argue by iteration on the dimension $i=$ $3 \ldots, n$. More precisely, if $F$ is any $i$-dimensional face of $\left[Q_{l, h}\right]_{i}$ with disjoint 
interior from both $\partial Q_{\rho}^{n}$ and $\partial Q_{\delta}^{n}$, we extend $w_{j}$ to the interior of $F$ by means of a suitable radial extension of the boundary datum of $w_{j}$ on $\partial F$ similar to the one in (4.30), so that

$$
E_{1,1}\left(w_{j}, F\right) \leq C \frac{\rho}{q} E_{1,1}\left(w_{j}, \partial F\right) .
$$

Therefore, by the construction, and for (4.27), we readily infer that

$$
E_{1,1}\left(w_{j}, Q_{\rho}^{n} \backslash Q_{\delta}^{n}\right) \leq C \sum_{i=1}^{n-1}\left(\frac{\rho}{q}\right)^{n-i} E_{1,1}\left(v_{j}, \Sigma_{\rho}^{i}\right),
$$

so that by (4.29) and (4.17) we obtain again, for $m>1 / \widehat{C}^{2}$,

$$
E_{1,1}\left(w_{j}, Q_{\rho}^{n} \backslash Q_{\delta}^{n}\right) \leq C \frac{1}{\varepsilon_{m} \cdot m} \mathcal{E}_{1,1}\left(T, \bar{B}_{2 r}\left(x_{0}\right) \times \mathcal{Y}\right) .
$$

Remark 4.4. For future use, we notice that for any $n \geq 3$ the function $w_{j}$ this way constructed is smooth on the closure of $Q_{\rho}^{n} \backslash Q_{\delta}^{n}$, up to a "smooth" closed $(n-3)$-dimensional set. This yields that the graph of $w_{j}$ has no boundary in the interior of $Q_{\rho}^{n} \backslash Q_{\delta}^{n}$, i.e.,

$$
\partial G_{w_{j}}=0 \quad \text { on } \quad \mathcal{Z}^{n-1,1}\left(\operatorname{int}\left(Q_{\rho}^{n} \backslash Q_{\delta}^{n}\right) \times \mathcal{Y}\right) .
$$

We finally set for any $n \geq 3$

$$
\widetilde{w}_{\varepsilon}^{(j)}(x):=\left\{\begin{array}{lll}
w_{\varepsilon}^{(j)}(x) & \text { if } & x \in Q_{\delta}^{n} \\
w_{j}(x) & \text { if } & x \in Q_{\rho}^{n} \backslash Q_{\delta}^{n}
\end{array}\right.
$$

and define $u_{k}^{(j)}: \bar{B}_{r}\left(x_{0}\right) \rightarrow \mathcal{Y}$ by

$$
u_{k}^{(j)}(x):=\left\{\begin{array}{lll}
\widetilde{w}_{\varepsilon_{k}}^{(j)} \circ \psi_{j}(x) & \text { if } & x \in \bar{B}_{\rho}\left(x_{0}\right) \\
w_{k}^{(j)}(x) & \text { if } & x \in \bar{B}_{r}\left(x_{0}\right) \backslash \bar{B}_{\rho}\left(x_{0}\right),
\end{array}\right.
$$

where $\rho=\rho_{k}$ and $\varepsilon_{k} \searrow 0$ along a sequence.

Step 5: Approximating maps on the whole domain. For any $n \geq 2$ we define now $u_{k}^{(m)}: B^{n} \rightarrow \mathcal{Y}$ by

$$
u_{k}^{(m)}(x):=\left\{\begin{array}{lll}
u_{k}^{(j)}(x) & \text { if } & x \in B_{j}, \quad j \in \mathbb{N} \\
u_{T}(x) & \text { if } \quad x \in B^{n} \backslash \Omega_{m},
\end{array} \quad \Omega_{m}:=\bigcup_{j=1}^{\infty} B_{j} .\right.
$$

By Step 4 we know that $u_{k}^{(j)} \in W^{1,1}\left(B_{j}, \mathcal{Y}\right)$ for every $j$ and $k$. Moreover, by (4.6), and since $u_{k}^{(j)}=u_{T}$ on $\partial B_{j}$ for every $j$, we infer that $u_{k}^{(m)}$ is for every $k$ a function in $B V\left(B^{n}, \mathcal{Y}\right)$, with null Cantor part, $\left|D^{C} u_{k}^{(m)}\right|=0$. 
We now deal with the energy estimates of $u_{k}^{(m)}$, first considering the simpler case $n=2$.

The case $n=2$. By (4.19) and Step 3 we infer that

$$
\limsup _{k \rightarrow \infty} E_{1,1}\left(u_{k}^{(m)}, \Omega_{m}\right) \leq\left(\operatorname{Lip} \Pi_{\varepsilon_{m}}\right)^{2} \cdot\left|D u_{T}\right|\left(\Omega_{m}\right),
$$

whereas by (4.6)

$$
\left|D u_{T}\right|\left(\Omega_{m}\right) \leq \mu_{d}\left(\Omega_{m}\right)+\frac{1}{m} .
$$

By a diagonal argument, setting $u_{m}:=u_{k_{m}}^{(m)}$ for a suitable sequence $k_{m} \rightarrow \infty$ as $m \rightarrow \infty$, we infer that

$$
\lim _{m \rightarrow \infty}\left|D u_{m}\right|\left(B^{2}\right)=\left|D u_{T}\right|\left(B^{2}\right) .
$$

The case $n \geq 3$. By (4.31) and (4.32) we infer that

$$
\sum_{j=1}^{\infty} E_{1,1}\left(u_{k}^{(m)}, \psi_{j}^{-1}\left(Q_{\rho}^{n} \backslash Q_{\delta}^{n}\right)\right) \leq C \frac{1}{\varepsilon_{m} \cdot m} \sum_{j=1}^{\infty} \mathcal{E}_{1,1}\left(T, \widetilde{B}_{j} \times \mathcal{Y}\right),
$$

whereas by Theorem 4.1, on account of (4.3), we obtain

$$
\sum_{j=1}^{\infty} \mathcal{E}_{1,1}\left(T, \widetilde{B}_{j} \times \mathcal{Y}\right) \leq C \cdot\left(\mathcal{E}_{1,1}\left(T, B^{n} \times \mathcal{Y}\right)+\mathcal{L}^{n}\left(B^{n}\right)\right)<\infty,
$$

and $1 /\left(\varepsilon_{m} \cdot m\right) \rightarrow 0$ as $m \rightarrow \infty$, see Remark 4.2. On the other hand, by (4.24), and since $\eta \rightarrow 0$ as $m \rightarrow \infty$ in (4.21), as in the case $n=2$ we estimate the energy of $u_{k}^{(m)}$ on the sets $\psi_{j}^{-1}\left(Q_{\delta}^{n}\right)$. In particular, setting $u_{m}:=u_{k_{m}}^{(m)}$ for suitable sequence $k_{m} \rightarrow \infty$ as $m \rightarrow \infty$, we infer that

$$
\lim _{m \rightarrow \infty} \sum_{j=1}^{\infty} E_{1,1}\left(u_{m}, \psi_{j}^{-1}\left(Q_{\delta}^{n}\right)\right)=\mu_{d}\left(B^{n}\right)
$$

and hence, by Step 3, that for any $n \geq 2$

$$
\lim _{m \rightarrow \infty}\left|D u_{m}\right|\left(B^{n}\right)=\left|D u_{T}\right|\left(B^{n}\right) .
$$

Moreover, in any dimension $n \geq 2$, since for every $j$ the radius of the ball $B_{j}$ in $\mathcal{F}_{m}^{\prime}$ is smaller than $1 / m$, and $u_{k}^{(m)}=u_{T}$ on $\partial B_{j}$, the above energy estimates and the Poincaré inequality yield that for $m$ sufficiently large

$$
\begin{aligned}
\int_{B^{n}}\left|u_{m}-u_{T}\right| d x & =\sum_{j=1}^{\infty} \int_{B_{j}}\left|u_{k_{m}}^{(m)}-u_{T}\right| d x \leq \sum_{j=1}^{\infty} C_{n} \cdot \frac{1}{m} \cdot\left|D u_{T}\right|\left(B_{j}\right) \\
& \leq C_{n} \cdot \frac{1}{m} \cdot\left|D u_{T}\right|\left(B^{n}\right),
\end{aligned}
$$


where $C_{n}>0$ is an absolute constant. This proves the $L^{1}$-convergence of $u_{m}$ to $u_{T}$ as $m \rightarrow \infty$, and hence weakly in the $B V$-sense.

Finally, for future use, we observe that by the definition of $u_{m}$, on account of (4.6), the previous construction yields that the jump part of $D u_{m}$ strictly converges to the jump part of $D u_{T}$. Therefore, denoting by

$$
\widetilde{D} u_{m}:=D^{a} u_{m}+D^{C} u_{m}, \quad \widetilde{D} u_{T}:=D^{a} u_{T}+D^{C} u_{T},
$$

the diffuse part of $D u_{m}$ and $D u_{T}$, where we recall that the Cantor part $\left|D^{C} u_{m}\right|\left(B^{n}\right)=$ 0 for every $m$, by (4.34) we have

$$
\widetilde{D} u_{m} \rightarrow \widetilde{D} u_{T} \quad \text { and } \quad\left|\widetilde{D} u_{m}\right|\left(B^{n}\right) \rightarrow\left|\widetilde{D} u_{T}\right|\left(B^{n}\right) .
$$

Step 6: Approximating currents. For every $m$ and $k$ let $T_{k}^{(m)} \in \mathcal{D}_{n, 1}\left(B^{n} \times \mathcal{Y}\right)$ be given by

$$
T_{k}^{(m)}:=\sum_{j=1}^{\infty} G_{u_{k}^{(j)}}\left\llcorner\operatorname{int}\left(B_{j}\right) \times \mathcal{Y}+T\left\llcorner\left(B^{n} \backslash \Omega_{m}\right) \times \mathcal{Y},\right.\right.
$$

where $u_{k}^{(j)} \in W^{1,1}\left(B_{j}, \mathcal{Y}\right)$ is defined by (4.33). Since the boundary $\partial G_{u_{k}^{(j)}}\left\llcorner\operatorname{int}\left(B_{j}\right) \times\right.$ $\mathcal{Y}=0$, whereas

$$
\partial\left(G_{u_{k}^{(j)}}\left\llcorner\operatorname{int}\left(B_{j}\right) \times \mathcal{Y}\right)=\left\langle T, d_{x_{0}}, r\right\rangle,\right.
$$

we readily infer that $T_{k}^{(m)} \in \operatorname{cart}^{1,1}\left(B^{n} \times \mathcal{Y}\right)$, with corresponding function in $B V\left(B^{n}, \mathcal{Y}\right)$ given by $u_{k}^{(m)}$, see (4.33). Setting $T_{m}:=T_{k_{m}}^{(m)}$, where the sequence $k_{m} \rightarrow \infty$ is defined as in Step 5, by (4.6) and (4.35) we readily infer that

$$
\lim _{m \rightarrow \infty} \mathcal{E}_{1,1}\left(T_{m}, \Omega_{m} \times \mathcal{Y}\right)=\left|\widetilde{D} u_{T}\right|\left(B^{n}\right)
$$

which clearly yields that

$$
\lim _{m \rightarrow \infty} \mathcal{E}_{1,1}\left(T_{m}, B^{n} \times \mathcal{Y}\right)=\mathcal{E}_{1,1}\left(T, B^{n} \times \mathcal{Y}\right)
$$

It therefore remains to show that, possibly taking a subsequence,

$$
T_{m} \rightarrow T \quad \text { weakly in } \mathcal{Z}_{n, 1}\left(B^{n} \times \mathcal{Y}\right)
$$

By applying Theorem 2.15, the proof of which is independent of the one of Theorem 2.14, every $T_{m}$ is the weak limit of a sequence of smooth graphs of maps $v_{k}^{(m)} \in C^{1}\left(B^{n}, \mathcal{Y}\right)$, with energies converging to the energy of $T_{m}$. Therefore, since $\sup _{m} \mathcal{E}_{1,1}\left(T_{m}, B^{n} \times \mathcal{Y}\right)<\infty$, arguing as in the first part of Section 2, by a diagonal argument we may and do assume that, possibly passing to a subsequence, $T_{m}$ 
weakly converges in $\mathcal{Z}_{n, 1}\left(B^{n} \times \mathcal{Y}\right)$ to some current $\widetilde{T} \in$ cart $^{1,1}\left(B^{n} \times \mathcal{Y}\right)$. Similarly, by the lower semicontinuity theorem for smooth graphs, Theorem 2.12, we infer that for any open set $A \subset B^{n}$ we have

$$
\mathcal{E}_{1,1}(\widetilde{T}, A \times \mathcal{Y}) \leq \liminf _{m \rightarrow \infty} \mathcal{E}_{1,1}\left(T_{m}, A \times \mathcal{Y}\right) .
$$

Moreover, since the sequence of functions $\left\{u_{m}\right\} \subset B V\left(B^{n}, \mathcal{Y}\right)$ corresponding to the $T_{m}$ 's weakly converges in the $B V$-sense to $u_{T} \in B V\left(B^{n}, \mathcal{Y}\right)$, we infer that $u_{T}$ is the $B V$-function corresponding to $\widetilde{T}$.

We first show that $\widetilde{T}$ agrees with $T$ on $\Omega \times \mathcal{Y}$, where

$$
\Omega:=B^{n} \backslash J_{C}(T),
$$

$J_{c}(T)$ being the set of points of jump-concentration of $T$. Fix $m_{0} \in \mathbb{N}$. Since

$$
\Omega \subset \Omega_{m} \subset A_{m}, \quad A_{m}:=B^{n} \backslash J_{m},
$$

and $\left\{J_{m}\right\}$ is an increasing sequence of closed sets, for any $m \geq m_{0}$ we infer that

$$
A_{m_{0}}=\Omega_{m} \cup\left[\left(J_{c}(T) \backslash J_{m_{0}}\right) \backslash \Omega_{m}\right],
$$

with disjoint union. Moreover, we recall that $T_{m}$ is equal to $T$ out of $\Omega_{m} \times \mathcal{Y}$. Therefore, since by (4.6)

$$
\mathcal{E}_{1,1}\left(T,\left[\left(J_{c}(T) \backslash J_{m_{0}}\right) \backslash \Omega_{m}\right] \times \mathcal{Y}\right) \leq \frac{1}{m_{0}},
$$

by (4.38) and (4.36) we obtain

$$
\begin{aligned}
\mathcal{E}_{1,1}\left(\widetilde{T}, A_{m_{0}} \times \mathcal{Y}\right) & \leq\left|\widetilde{D} u_{T}\right|\left(B^{n}\right)+\liminf _{m \rightarrow \infty} \mathcal{E}_{1,1}\left(T_{m},\left[\left(J_{c}(T) \backslash J_{m_{0}}\right) \backslash \Omega_{m}\right] \times \mathcal{Y}\right) \\
& \leq\left|\widetilde{D} u_{T}\right|\left(B^{n}\right)+\liminf _{m \rightarrow \infty} \mathcal{E}_{1,1}\left(T,\left[\left(J_{c}(T) \backslash J_{m_{0}}\right) \backslash \Omega_{m}\right] \times \mathcal{Y}\right) \\
& \leq\left|\widetilde{D} u_{T}\right|\left(B^{n}\right)+1 / m_{0} .
\end{aligned}
$$

By outer regularity, since $\left|\widetilde{D} u_{T}\right|\left(J_{c}(T)\right)=0$ and $A_{m} \searrow \Omega$ as $m \rightarrow \infty$, we infer that

$$
\mathcal{E}_{1,1}(\widetilde{T}, \Omega \times \mathcal{Y}) \leq\left|\widetilde{D} u_{T}\right|(\Omega) .
$$

Therefore, decomposing the energy of $\widetilde{T}$ into its diffuse and jump-concentration part, see (4.3), we infer that the jump-concentration part is concentrated in the jumpconcentration set of $T$, so that

$$
J_{c}(\tilde{T}) \subset J_{c}(T) \quad \text { and } \quad \tilde{T}\llcorner\Omega \times \mathcal{Y}=T\llcorner\Omega \times \mathcal{Y} .
$$

We now show that $\widetilde{T}$ agrees with $T$ on $J_{c}(T) \times \mathcal{Y}$, which concludes the proof. As before, since $T_{m}$ is equal to $T$ out of $\Omega_{m} \times \mathcal{Y}$, and $\Omega_{m} \cap J_{m_{0}}=\emptyset$ if $m \geq m_{0}$, for every form $\omega \in \mathcal{Z}^{n, 1}\left(B^{n} \times \mathcal{Y}\right)$ we have

$$
\begin{aligned}
\left((\widetilde{T}-T)\left\llcorner J_{m_{0}} \times \mathcal{Y}\right)(\omega)\right. & =\left(\left(\widetilde{T}-T_{m}\right)\left\llcorner J_{m_{0}} \times \mathcal{Y}\right)(\omega)+\left(\left(T_{m}-T\right)\left\llcorner J_{m_{0}} \times \mathcal{Y}\right)(\omega)\right.\right. \\
& =\left(\left(\widetilde{T}-T_{m}\right)\left\llcorner J_{m_{0}} \times \mathcal{Y}\right)(\omega) \rightarrow 0\right.
\end{aligned}
$$


as $m \rightarrow \infty$, by the weak convergence of $T_{m}$ to $\widetilde{T}$. This yields that

$$
\widetilde{T}\left\llcorner J_{m_{0}} \times \mathcal{Y}=T\left\llcorner J_{m_{0}} \times \mathcal{Y}\right.\right.
$$

and finally the assertion, by inner regularity, since $J_{m} \nearrow J_{c}(T)$ in the $\mathcal{H}^{n-1}$-sense as $m \rightarrow \infty$.

\section{The density theorem: part II}

In this section we prove Theorem 2.15. Extending the notation from the previous section, see (4.3), in the sequel for every current $\widetilde{T} \in \operatorname{cart}^{1,1}\left(B^{n} \times \mathcal{Y}\right)$ we will denote by $\mu_{J c, \widetilde{T}}$ the Radon measure on $B^{n}$ given for every Borel set $B \subset B^{n}$ by

$$
\mu_{J c, \widetilde{T}}(B):=\int_{J_{c}(\widetilde{T}) \cap B} \mathcal{L}_{\widetilde{T}}(x) d \mathcal{H}^{n-1}(x),
$$

that corresponds to the jump-concentration part of the $B V$-energy $\mathcal{E}_{1,1}(\widetilde{T}, B \times \mathcal{Y})$. We also recall that if $\widetilde{T} \in \operatorname{cart}^{1,1}\left(B^{n} \times \mathcal{Y}\right)$ satisfies $\left|D^{C} u_{\widetilde{T}}\right|=0$, for every Borel set $B \subset B^{n}$

$$
\mathcal{E}_{1,1}(\widetilde{T}, B \times \mathcal{Y})=\int_{B}\left|\nabla u_{\widetilde{T}}(x)\right| d x+\mu_{J c, \widetilde{T}}(B) .
$$

Moreover, for any $\widetilde{T}$ as above, in this section we will denote by $\mathbf{F}(\widetilde{T})$ the flat norm given by

$$
\mathbf{F}(\widetilde{T}):=\sup \left\{\widetilde{T}(\phi) \mid \phi \in \mathcal{Z}^{n-1}\left(B^{n} \times \mathcal{Y}\right), \mathbf{F}(\phi) \leq 1\right\},
$$

where

$$
\mathbf{F}(\phi):=\max \left\{\sup _{z \in B^{n} \times \mathcal{Y}}\|\phi(z)\|, \sup _{z \in B^{n} \times \mathcal{Y}}\|d \phi(z)\|\right\},
$$

and we notice that the flat convergence $\mathbf{F}\left(T_{k}-T\right) \rightarrow 0$ yields the weak convergence $T_{k} \rightarrow T$ weakly in $\mathcal{Z}_{n, 1}\left(\widetilde{B}^{n} \times \mathcal{Y}\right)$, compare [22].

Proof of Theorem 2.15. It is based on the following:

Proposition 5.1. Let $\widetilde{T} \in \operatorname{cart}^{1,1}\left(B^{n} \times \mathcal{Y}\right)$ be such that $\left|D^{C} u_{\widetilde{T}}\right|\left(B^{n}\right)=0$. Let $\varepsilon \in(0,1 / 2)$ and $k \in \mathbb{N}$. We can find a current $\widehat{T} \in \operatorname{cart}^{1,1}\left(B^{n} \times \mathcal{Y}\right)$ such that

$$
\begin{aligned}
& \mathcal{E}_{1,1}\left(\widehat{T}, B^{n} \times \mathcal{Y}\right) \leq \mathcal{E}_{1,1}\left(\widetilde{T}, B^{n} \times \mathcal{Y}\right)+\varepsilon^{k}, \\
& \mu_{J c, \widehat{T}}\left(B^{n}\right) \leq \frac{1}{2} \cdot \mu_{J c, \widetilde{T}}\left(B^{n}\right) \quad \text { and }\left|D^{C} u_{\widehat{T}}\right|=0 .
\end{aligned}
$$


In fact, for any $\varepsilon \in(0,1 / 2)$ we apply iteratively Proposition 5.1 as follows. Letting $T_{0}^{\varepsilon}:=T$, at the $k^{\text {th }}$ step, in correspondence of $\widetilde{T}:=T_{k-1}^{\varepsilon}$ we find $\widehat{T}:=$ $T_{k}^{\varepsilon}$ such that (5.2) holds true. By induction on $k \in \mathbb{N}$, we define $T^{\varepsilon}:=T_{\infty}^{\varepsilon} \in$ $\operatorname{cart}^{1,1}\left(B^{n} \times \mathcal{Y}\right)$ such that

$$
\mathcal{E}_{1,1}\left(T^{\varepsilon}, B^{n} \times \mathcal{Y}\right) \leq \mathcal{E}_{1,1}\left(T, B^{n} \times \mathcal{Y}\right)+\sum_{k=1}^{\infty} \varepsilon^{k} \leq \mathcal{E}_{1,1}\left(T, B^{n} \times \mathcal{Y}\right)+2 \varepsilon
$$

and $\left|D^{C} u_{T^{\varepsilon}}\right|=0$. Moreover, since for every $k$

$$
\mu_{J c, T_{k}^{\varepsilon}}\left(B^{n}\right) \leq 2^{-k} \cdot \mu_{J c, T}\left(B^{n}\right),
$$

letting $k \rightarrow \infty$ we obtain that $\mu_{J c, T^{\varepsilon}}\left(B^{n}\right)=0$. Finally, since

$$
\mathbf{F}\left(T^{\varepsilon}-T\right) \leq \sum_{k=1}^{\infty} \mathbf{F}\left(T_{k}^{\varepsilon}-T_{k-1}^{\varepsilon}\right) \leq \sum_{k=1}^{\infty} \varepsilon^{k} \leq 2 \varepsilon,
$$

letting $T_{k}:=T^{\varepsilon_{k}}$ for some sequence $\varepsilon_{k} \searrow 0$, and $u_{k}:=u_{T_{k}}$, we infer that the sequence $\left\{T_{k}\right\} \subset$ cart $^{1,1}\left(B^{n} \times \mathcal{Y}\right)$ weakly converges to $T$ with $\mathcal{E}_{1,1}\left(T_{k}\right) \rightarrow$ $\mathcal{E}_{1,1}(T)$ as $k \rightarrow \infty$. Moreover, since $\left|D^{C} u_{k}\right|\left(B^{n}\right)=0$ and $\mu_{J c, T_{k}}\left(B^{n}\right)=0$ for every $k$, we obtain that $u_{k} \in W^{1,1}\left(B^{n}, \mathcal{Y}\right)$ and that $T_{k}$ agrees with the current $G_{u_{k}}$ given by the integration of forms in $\mathcal{Z}^{n, 1}\left(B^{n} \times \mathcal{Y}\right)$ over the rectifiable graph of $u_{k}$, see (2.1), so that $\mathcal{E}_{1,1}\left(T_{k}\right)=\mathcal{E}_{1,1}\left(u_{k}\right)$.

By means of Bethuel's density theorem [5], for every $k$ we find a smooth sequence $\left\{u_{h}^{(k)}\right\}_{h} \subset C^{1}\left(B^{n}, \mathcal{Y}\right)$ that strongly converges to $u_{k}$ in the $W^{1,1}$-sense as $h \rightarrow \infty$. In fact, even if the first homotopy group $\pi_{1}(\mathcal{Y})$ is non-trivial, being commutative it is homeomorphic to the first homology group $H_{1}(\mathcal{Y})$. Therefore, the null-boundary condition

$$
\partial G_{u_{k}}=0 \quad \text { on } \quad \mathcal{Z}^{n-1,1}\left(B^{n} \times \mathcal{Y}\right)
$$

allows to remove the $(n-2)$-dimensional singularities, compare [6] and e.g. [16]. Lower dimensional singularities are removed as in [5]. Since the strong convergence yields $G_{u_{h}^{(k)}} \rightarrow G_{u_{k}}$ with $\mathcal{E}_{1,1}\left(u_{h}^{(k)}\right) \rightarrow \mathcal{E}_{1,1}\left(u_{k}\right)$, the assertion follows by means of a diagonal argument.

Remark 5.2. This is the exact point where the commutativity hypothesis on the first homotopy group $\pi_{1}(\mathcal{Y})$ is used, in addition to (5.3). If $\pi_{1}(\mathcal{Y})$ is non-abelian, even in dimension $n=2$ we find functions $u \in W^{1,1}\left(B^{2}, \mathcal{Y}\right)$, smooth outside the origin and satisfying (5.3), such that for every sequence of smooth maps $u_{h}$ : $B^{n} \rightarrow \mathcal{Y}$ for which $G_{u_{h}} \rightarrow G_{u}$ weakly in $\mathcal{Z}_{n, 1}\left(B^{n} \times \mathcal{Y}\right)$ we have

$$
\liminf _{h \rightarrow \infty} \int_{B^{2}}\left|D u_{h}\right| d x \geq C+\int_{B^{2}}|D u| d x
$$

for some absolute constant $C>0$, compare [17]. 
Proof of Proposition 5.1. We set $\widetilde{T}=T$, for simplicity, and divide the proof in four steps.

Step 1: Blow-up argument. We apply the argument by Federer [9, 4.2.19]. The rectifiable measure $\mu_{J c, T}$ can be written as

$$
\mu_{J c, T}=\mathcal{L}_{T} \mathcal{H}^{n-1}\left\llcorner J_{c}(T),\right.
$$

where the jump-concentration set $J_{c}(T)$ is countably $\mathcal{H}^{n-1}$-rectifiable and the density $\mathcal{L}_{T}(x)$ is a non-negative $\mathcal{H}^{n-1}\left\llcorner J_{c}(T)\right.$-summable function on $J_{c}(T)$. Therefore, by $[9,3.2 .29]$ there exists a countable family $\mathcal{G}$ of $(n-1)$-dimensional $C^{1}$ submanifolds $\mathcal{M}_{j}$ of $B^{n}$ such that $\mu_{J c, T}$-almost all of $B^{n}$ is covered by $\mathcal{G}$. Moreover, since $\mu_{J c, T}\left(B^{n}\right)<\infty$, we can find a positive number $\theta>0$ so that the subset

$$
J:=\left\{x \in J_{c}(T) \mid \mathcal{L}_{T}(x)>\theta\right\}
$$

satisfies the following properties:

$$
\mathcal{H}^{n-1}(J)<\infty \quad \text { and } \quad \mu_{J c}\left(B^{n} \backslash J\right)<\frac{1}{4} \cdot \mu_{J c, T}\left(B^{n}\right) .
$$

Let $\sigma>0$ to be fixed. By [9, 2.10.19], by the Vitali-Besicovitch theorem, Theorem 3.2, and by the properties of the class $\operatorname{cart}^{1,1}\left(B^{n} \times \mathcal{Y}\right)$ we can find a number $t_{\sigma} \in(0,1)$, a countable disjoint family of closed balls $B_{j}$, contained in $B^{n}$ and centered at points in $J$, and a bilipschitz homeomorphism $\psi_{\sigma}$ from $B^{n}$ onto itself satisfying the properties listed below, where $c>0$ is an absolute constant, possibly varying from line to line, which is independent of $\sigma$ and of the radii $r_{j}$ of the balls $B_{j}$.

i) $\mu_{J c, T}\left(B^{n} \backslash \bigcup_{j} B_{j}\right)=0$.

ii) If $B_{j}:=\bar{B}\left(p_{j}, r_{j}\right)$, for every $j$ there is a manifold $\mathcal{M}_{j}$ of $\mathcal{G}$ such that $p_{j} \in \mathcal{M}_{j}$.

iii) Since $\mathcal{H}^{n-1}(J)<\infty$, then

$$
\sum_{j=1}^{\infty} r_{j}^{n-1} \leq c \cdot \mathcal{H}^{n-1}(J)<\infty .
$$

iv) Letting $C_{j}:=B\left(p_{j}, t_{\sigma} r_{j}\right) \cap \mathcal{M}_{j}$, we have

$$
\mu_{J c, T}\left(B\left(p_{j}, r_{j}\right) \backslash C_{j}\right) \leq \sigma \cdot \mu_{J c, T}\left(B\left(p_{j}, r_{j}\right)\right) \quad \forall j .
$$

v) If $p_{j} \notin J_{u_{T}}$, it is a Lebesgue point of $u_{T}$ whereas, if $p_{j} \in J_{u_{T}}$, the one-sided approximate limits of $u_{T}$ at $p_{j}$ are well-defined.

vi) The 1-dimensional restriction $\widehat{\pi}_{\#}\left(T\left\llcorner\left\{p_{j}\right\} \times \mathcal{Y}\right)\right.$ is well-defined, compare Definition 2.8 , and

$$
\widehat{\pi}_{\#}\left(T\left\llcorner\left\{p_{j}\right\} \times \mathcal{Y}\right)=\Gamma_{j}\right.
$$

for some integral chain $\Gamma_{j} \in \mathcal{D}_{1}(\mathcal{Y})$. 
vii) If $\eta_{p_{j}, \lambda}: \mathbb{R}^{n} \times \mathbb{R}^{N} \rightarrow \mathbb{R}^{n} \times \mathbb{R}^{N}$ denotes the "blow-up" map $\eta_{p_{j}, \lambda}(x, y):=$ $\left(\frac{x-p_{j}}{\lambda}, y\right)$, the limit current

$$
S_{j}(\omega):=\lim _{\lambda \rightarrow 0^{+}} \eta_{p_{j}, \lambda \# T} T(\omega), \quad \omega \in \mathcal{Z}^{n, 1}\left(B^{n} \times \mathcal{Y}\right)
$$

is well-defined, and the flat distance of $T$ from $S_{j}$ is small on $B_{j} \times \mathcal{Y}$, i.e.

$$
\mathbf{F}\left(S _ { j } \left\llcornerB_{j} \times \mathcal{Y}-T\left\llcorner B_{j} \times \mathcal{Y}\right) \leq c \cdot \sigma \cdot r_{j}^{n-1} .\right.\right.
$$

viii) Since $\left|D u_{T}\right|(B) \leq \mu_{T}(B)$, we have

$$
\frac{\left|D u_{T}\right|\left(B\left(p_{j}, r_{j}\right) \backslash C_{j}\right)}{\omega_{n-1} r_{j}^{n-1}} \leq c \cdot \sigma,
$$

where $\omega_{n-1}$ is the measure of the $(n-1)$-dimensional unit ball.

ix) Since $\mathcal{L}_{T}\left(p_{j}\right)$ is the $(n-1)$-dimensional density of $\mu_{J c, T}$ at $p_{j}$, we have

$$
\left|\mu_{J c, T}\left(B_{j}\right)-\mathcal{L}_{T}\left(p_{j}\right) \cdot \omega_{n-1} r_{j}{ }^{n-1}\right| \leq \sigma \cdot \omega_{n-1} r_{j}{ }^{n-1} .
$$

x) $\operatorname{Lip} \psi_{\sigma} \leq 2$ and $\operatorname{Lip} \psi_{\sigma}^{-1} \leq 2$. Moreover, $\psi_{\sigma}$ maps bijectively $B_{j}$ onto $B_{j}$, with $\psi_{\sigma \mid \partial B_{j}}=I d_{\mid \partial B_{j}}$ and $\psi_{\sigma}\left(p_{j}\right)=p_{j}$ for all $j$, and $\psi_{\sigma}$ is equal to the identity outside the union of the balls $B_{j}$.

xi) $\psi_{\sigma}\left(C_{j}\right)=B\left(p_{j}, \rho_{j}\right) \cap\left(p_{j}+\operatorname{Tan}\left(\mathcal{M}_{j}, p_{j}\right)\right)$ for every $j$, where $\operatorname{Tan}\left(\mathcal{M}_{j}, p_{j}\right)$ is the $(n-1)$-dimensional tangent space to $\mathcal{M}_{j}$ at $p_{j}$ and $\rho_{j} \in\left(r_{j} / 2, r_{j}\right)$.

As a consequence, defining $T_{j}^{\sigma} \in \mathcal{D}_{n, 1}\left(\operatorname{int}\left(B_{j}\right) \times \mathcal{Y}\right)$ for any $j$ by

$$
T_{j}^{\sigma}:=\left(\psi_{\sigma} \bowtie \operatorname{Id}_{\mathbb{R}^{N}}\right)_{\#}\left(T\left\llcorner\operatorname{int}\left(B_{j}\right) \times \mathcal{Y}\right),\right.
$$

we infer that $T_{j}^{\sigma}$ belongs to $\operatorname{cart}^{1,1}\left(\operatorname{int}\left(B_{j}\right) \times \mathcal{Y}\right)$ and its corresponding function $u_{j}^{\sigma}:=u_{T_{j}^{\sigma}} \in B V\left(\operatorname{int}\left(B_{j}\right), \mathcal{Y}\right)$ is given by

$$
u_{j}^{\sigma}:=\left(u_{T} \circ \psi_{\sigma}^{-1}\right)_{\mid \operatorname{int}\left(B_{j}\right)} .
$$

Moreover, we clearly have

$$
\mu_{J c, T_{j}^{\sigma}}=\psi_{\sigma \#}\left(\mu_{J c, T}\left\llcorner\operatorname{int}\left(B_{j}\right)\right) .\right.
$$

Step 2: Approximation on the balls $B_{j}$. We now apply for every $j$ a "dipole construction" to approximate almost all the Jump-concentration part of $T_{j}^{\sigma}$. Set

$$
x=\left(\widetilde{x}, x_{n}\right) \in \mathbb{R}^{n-1} \times \mathbb{R} .
$$


Without loss of generality we may and will assume that

$$
B_{j}=\bar{B}_{R}^{n}, \quad B\left(p_{j}, \rho_{j}\right)=B_{r}^{n}, \quad 0<r<R,
$$

where $B_{r}^{n}:=B^{n}(0, r)$, so that $R=r_{j}$ and $r=\rho_{j}$, and

$$
B\left(p_{j}, \rho_{j}\right) \cap\left(p_{j}+\operatorname{Tan}\left(\mathcal{M}_{j}, p_{j}\right)\right)=D_{r} \times\{0\} \subset \mathbb{R}^{n-1} \times \mathbb{R}, \quad D_{r}:=B^{n-1}\left(0_{\mathbb{R}^{n-1}}, r\right) .
$$

Let $y(\widetilde{x}):=(r-|\widetilde{x}|)$ denote the distance of $\tilde{x}$ from the boundary of the $(n-1)$ disk $D_{r}$. For $\delta>0$ small, let

$$
\phi_{\delta}(x):=\left(\tilde{x}, \varphi_{\delta}(y(\tilde{x})) x_{n}\right), \quad x \in D_{r} \times[-1,1], \quad \varphi_{\delta}(y):=\min \{y, \delta\} .
$$

Let $\Omega_{\delta}:=\phi_{\delta}\left(D_{r} \times[-1,1]\right)$ be the "neighborhood" of $D_{r} \times\{0\}$ in $B_{R}^{n}$ given by

$$
\Omega_{\delta}=\left\{\left(\tilde{x}, x_{n}\right) \mid \tilde{x} \in D_{r}, \quad \rho \leq \varphi_{\delta}(y(\tilde{x}))\right\},
$$

where $\rho:=\left|x_{n}\right|$, and let

$$
\widetilde{\Omega}_{\delta}:=\phi_{\delta}\left(D_{r} \times[-1 / 2,1 / 2]\right)=\left\{\left(\tilde{x}, x_{n}\right) \mid \tilde{x} \in D_{r}, \rho \leq \varphi_{\delta}(y(\tilde{x})) / 2\right\} .
$$

Also, set

$$
\Omega_{(r, \delta)}:=\Omega_{\delta} \backslash\left(D_{r} \times\{0\}\right) .
$$

Let $v_{j}^{\sigma}:\left(\Omega_{\delta} \backslash \widetilde{\Omega}_{\delta}\right) \rightarrow \mathcal{Y}$ be given by $v_{j}^{\sigma}(x):=u_{j}^{\sigma} \circ \psi_{j}^{\sigma}(x)$, where $\psi_{j}^{\sigma}: \Omega_{\delta} \backslash \widetilde{\Omega}_{\delta} \rightarrow$ $\Omega_{(r, \delta)}$ is the bijective map

$$
\psi_{j}^{\sigma}\left(\tilde{x}, x_{n}\right):=\left(\tilde{x},\left(2-\frac{\varphi_{\delta}(y(\tilde{x}))}{\rho}\right) x_{n}\right) .
$$

Since we have

$$
\left|\nabla v_{j}^{\sigma}(x)\right| \leq c\left|\nabla u_{j}^{\sigma}\left(\widetilde{x},\left(2-\varphi_{\delta}(y(\widetilde{x})) / \rho\right) x_{n}\right)\right| \cdot\left(1+\varphi_{\delta}(y(\widetilde{x})) / \rho\right),
$$

and $\left.\left.\varphi_{\delta}(y(\tilde{x})) / \rho \in\right] 1 / 2,1\right]$, we infer that $v_{j}^{\sigma} \in B V\left(\Omega_{\delta} \backslash \widetilde{\Omega}_{\delta}, \mathcal{Y}\right)$, with

$$
\int_{\Omega_{\delta} \backslash \widetilde{\Omega}_{\delta}}\left|\nabla v_{j}^{\sigma}\right| d x \leq c \int_{\Omega_{\delta}}\left|\nabla u_{j}^{\sigma}\right| d x .
$$

Moreover, the current

$$
\bar{T}_{j}^{\sigma}:=\left(\left(\psi_{j}^{\sigma}\right)^{-1} \bowtie \operatorname{Id}_{\mathbb{R}^{N}}\right)_{\#}\left(T_{j}^{\sigma}\left\llcorner\left(\operatorname{int}\left(\Omega_{(r, \delta)}\right) \times \mathcal{Y}\right)\right)\right.
$$

belongs to $\operatorname{cart}^{1,1}\left(\operatorname{int}\left(\Omega_{\delta} \backslash \widetilde{\Omega}_{\delta}\right) \times \mathcal{Y}\right)$, its underlying $B V$-function is $v_{j}^{\sigma}$, and $\bar{T}_{j}^{\sigma}$ satisfies

$$
\mu_{J c, \bar{T}_{j}^{\sigma}}\left(\operatorname{int}\left(\Omega_{\delta} \backslash \widetilde{\Omega}_{\delta}\right)\right) \leq \mu_{J c, T_{j}^{\sigma}}\left(\operatorname{int}\left(\Omega_{(r, \delta)}\right)\right),
$$


so that by (5.6) we have

$$
\mu_{\bar{T}_{j}^{\sigma}}\left(\operatorname{int}\left(\Omega_{\delta} \backslash \widetilde{\Omega}_{\delta}\right)\right) \leq c \sigma \mu_{T_{j}^{\sigma}}\left(B_{r}^{n}\right) .
$$

We now define $w_{j}^{\sigma}:\left(\Omega_{\delta} \backslash \widetilde{\Omega}_{\delta}\right) \rightarrow \mathbb{R}^{N}$ by

$$
w_{j}^{\sigma}(x):=\left(\frac{2 \rho}{\varphi_{\delta}(y(\tilde{x}))}-1\right) \cdot v_{j}^{\sigma}\left(\tilde{x}, x_{n}\right)+\left(2-\frac{2 \rho}{\varphi_{\delta}(y(\tilde{x}))}\right) \cdot z_{j}^{ \pm},
$$

where \pm is the sign of $x_{n}$ and $z_{j}^{ \pm}$are the one-sided approximate limits of $u_{j}^{\sigma}$ at the point $0 \in J_{u_{j}^{\sigma}}$, so that

$$
\lim _{\rho \rightarrow 0^{+}} \rho^{-n} \int_{B_{\rho}^{ \pm}}\left|u_{j}^{\sigma}(x)-z_{j}^{ \pm}\right| d x=0
$$

if $p_{j}$ belongs to the jump set of $u_{j}^{\sigma}$, and they agree with the Lebesgue value of $u_{j}^{\sigma}$ at $p_{j}$, otherwise.

If $r-\delta \leq|\tilde{x}| \leq r$ and $(r-|\tilde{x}|) / 2<\rho<(r-|\tilde{x}|)$, then

$$
\left|\nabla w_{j}^{\sigma}\right|(x) \leq \frac{c}{r-|\widetilde{x}|}\left|v_{j}^{\sigma}(x)-z_{j}^{ \pm}\right|+c\left|\nabla v_{j}^{\sigma}(x)\right|,
$$

whereas if $|\tilde{x}| \leq r-\delta$ and $\delta / 2<\rho<\delta$, we estimate

$$
\left|\nabla w_{j}^{\sigma}\right|(x) \leq \frac{c}{\delta}\left|v_{j}^{\sigma}(x)-z_{j}^{ \pm}\right|+c\left|\nabla v_{j}^{\sigma}(x)\right| .
$$

Moreover, by (5.8) and the Poincaré inequality we infer that the oscillation of $u_{j}^{\sigma}$ on the upper and lower half-balls

$$
B_{r}^{ \pm}:=\left\{x \in B_{r}^{n} \mid \pm x_{n}>0\right\}
$$

is smaller than $c \sigma$, so that

$$
\left\|v_{j}^{\sigma}(x)-z_{j}^{ \pm}\right\|_{\infty, \Omega_{\delta} \backslash \widetilde{\Omega}_{\delta}} \leq c \sigma .
$$

As a consequence, on account of (5.10) we obtain

$$
\begin{aligned}
\int_{\Omega_{\delta} \backslash \widetilde{\Omega}_{\delta}}\left|\nabla w_{j}^{\sigma}\right| d x & \leq c \sigma \mathcal{L}^{n}\left(\Omega_{\delta} \backslash \widetilde{\Omega}_{\delta}\right)+c \int_{\Omega_{\delta} \backslash \widetilde{\Omega}_{\delta}}\left|\nabla v_{j}^{\sigma}\right| d x \\
& \leq c \sigma \mathcal{L}^{n}\left(\Omega_{\delta} \backslash \widetilde{\Omega}_{\delta}\right)+c \int_{\Omega_{\delta}}\left|\nabla u_{j}^{\sigma}\right| d x
\end{aligned}
$$

which is small if $\delta$ and $\sigma$ are small, by the absolute continuity. Also, since the oscillation of $w_{j}^{\sigma}$ is smaller than $c \sigma$, by projecting $w_{j}^{\sigma}$ into the manifold $\mathcal{Y}$, see 
Remark 1.9, we may and will assume that $w_{j}^{\sigma}$ is a function in $B V\left(\Omega_{\delta} \backslash \widetilde{\Omega}_{\delta}, \mathcal{Y}\right)$. We finally observe that

$$
w_{j}^{\sigma}\left(\tilde{x}, \pm \varphi_{\delta}(y(\tilde{x})) / 2\right)=z_{j}^{ \pm} \quad \forall \tilde{x} \in D_{r}
$$

Now, by means of the vertical part of the current $\bar{T}_{j}^{\sigma}$, we may and do define a current $\widetilde{T}_{j}^{\sigma} \in \operatorname{cart}^{1,1}\left(\operatorname{int}\left(\Omega_{\delta} \backslash \widetilde{\Omega}_{\delta}\right) \times \mathcal{Y}\right)$, with underlying $B V$-function $w_{j}^{\sigma}$, such that

$$
\mu_{J c, \widetilde{T}_{j}^{\sigma}}\left(\operatorname{int}\left(\Omega_{\delta} \backslash \widetilde{\Omega}_{\delta}\right)\right) \leq c \mu_{J c, \bar{T}_{j}^{\sigma}}\left(\operatorname{int}\left(\Omega_{\delta} \backslash \widetilde{\Omega}_{\delta}\right)\right)
$$

and $\widetilde{T}_{j}^{\sigma}$ satisfies the boundary condition

$$
\partial \widetilde{T}_{j}^{\sigma}=\partial T_{j}^{\sigma}\left\llcorner\partial \Omega_{\delta} \times \mathcal{Y}+\llbracket \partial \widetilde{\Omega}_{\delta} \cap B_{r}^{+} \rrbracket \times \delta_{z_{j}^{+}}-\llbracket \partial \widetilde{\Omega}_{\delta} \cap B_{r}^{-} \rrbracket \times \delta_{z_{j}^{-}} .\right.
$$

In particular, by (5.11) and (5.12), taking $\delta$ small, we infer that $\tilde{T}_{j}^{\sigma}$ satisfies the energy estimate

$$
\begin{aligned}
\mathcal{E}_{1,1}\left(\widetilde{T}_{j}^{\sigma}, \operatorname{int}\left(\Omega_{\delta} \backslash \widetilde{\Omega}_{\delta}\right) \times \mathcal{Y}\right) & =\int_{\substack{\Omega_{\delta} \backslash \widetilde{\Omega}_{\delta}\\
}}\left|\nabla w_{j}^{\sigma}\right| d x+\mu_{J c, \widetilde{T}_{j}^{\sigma}}\left(\operatorname{int}\left(\Omega_{\delta} \backslash \widetilde{\Omega}_{\delta}\right)\right) \\
& \leq c \sigma r^{n-1}+c \sigma \mu_{J c, T_{j}^{\sigma}}\left(B_{r}^{n}\right)
\end{aligned}
$$

Due to the property vi) above, setting

$$
\widehat{T}_{j}^{\sigma}:=\widetilde{T}_{j}^{\sigma}+T_{j}^{\sigma}\left\llcorner\left(B_{R}^{n} \backslash \Omega_{\delta}\right) \times \mathcal{Y},\right.
$$

we infer that $\widehat{T}_{j}^{\sigma}$ belongs to $\operatorname{cart}^{1,1}\left(\left(B_{R}^{n} \backslash \tilde{\Omega}_{\delta}\right) \times \mathcal{Y}\right)$, satisfies the boundary condition

$$
\begin{aligned}
\partial \widehat{T}_{j}^{\sigma}= & \partial T_{j}^{\sigma}\left\llcorner\partial B_{R}^{n} \times \mathcal{Y}-\llbracket \partial D_{r} \times\{0\} \rrbracket \times \Gamma_{j}\right. \\
& +\llbracket \partial \widetilde{\Omega}_{\delta} \cap B_{r}^{+} \rrbracket \times \delta_{z_{j}^{+}}-\llbracket \partial \widetilde{\Omega}_{\delta} \cap B_{r}^{-} \rrbracket \times \delta_{z_{j}^{-}}
\end{aligned}
$$

and the energy estimate

$$
\begin{aligned}
\mathcal{E}_{1,1}\left(\widehat{T}_{j}^{\sigma},\left(B_{R}^{n} \backslash \widetilde{\Omega}_{\delta}\right) \times \mathcal{Y}\right) \leq & \int_{B_{R}^{n}}\left|\nabla u_{j}^{\sigma}\right| d x \\
& +c \sigma r^{n-1}+c \sigma \mu_{J c, T_{j}^{\sigma}}\left(B_{r}^{n}\right) .
\end{aligned}
$$

To extend $\widehat{T}_{j}^{\sigma}$ to a current in $\operatorname{cart}^{1,1}\left(\operatorname{int}\left(B_{j}\right) \times \mathcal{Y}\right)$, we notice that $J_{c}\left(T_{j}^{\sigma}\right)=$ $\psi_{\sigma}\left(J_{c}(T) \cap \operatorname{int}\left(B_{j}\right)\right)$. Moreover, if $\gamma_{j} \in \Gamma_{T}\left(p_{j}\right)$ satisfies (1.7), of course $\gamma_{j}$ belongs to $\Gamma_{T_{j}^{\sigma}}\left(p_{j}\right)$ and satisfies

$$
\mathcal{L}\left(\gamma_{j}\right)=\mathcal{L}_{T_{j}^{\sigma}}\left(p_{j}\right)=\mathcal{L}_{T}\left(p_{j}\right)
$$


and $\gamma_{j \# \Perp} \llbracket(0,1) \rrbracket=\Gamma_{j}$, see property vi). We define $v_{j}^{\sigma}: \widetilde{\Omega}_{\delta} \rightarrow \mathcal{Y}$ by setting

$$
v_{j}^{\sigma}(x):=\gamma_{j}\left(\frac{1}{2}+\frac{x_{n}}{\varphi_{\delta}(y(\tilde{x}))}\right), \quad \tilde{x} \in D_{r}, \quad \rho \leq \varphi_{\delta}(y(\tilde{x})) / 2,
$$

where the orientation of $\gamma_{j}$ is chosen in such a way that $\gamma_{j}(0)=z_{j}^{-}$and $\gamma_{0}(1)=$ $z_{j}^{+}$, so that $\partial \llbracket \gamma_{j} \rrbracket=\delta_{z^{+}}-\delta_{z^{-}}$. Since

$$
v_{j}^{\sigma}(x):=\left(v \circ \phi_{\delta}^{-1}\right)(x), \quad x \in \phi_{\delta}\left(D_{r} \times[-1 / 2,1 / 2]\right),
$$

where $v: D_{r} \times[-1 / 2,1 / 2] \rightarrow \mathcal{Y}$ is given by $v(\widetilde{x}, t):=\tilde{\gamma}_{j}(1 / 2+t)$, we readily estimate

$$
\begin{aligned}
\int_{\widetilde{\Omega}_{\delta}}\left|D v_{j}^{\sigma}\right| d x & \leq \mathcal{L}\left(\gamma_{j}\right) \cdot\left(\mathcal{L}^{n-1}\left(D_{r-\delta}\right)+c \mathcal{L}^{n-1}\left(D_{r} \backslash D_{r-\delta}\right)\right) \\
& \leq \sigma r^{n-1}+\mathcal{L}^{n-1}\left(D_{r}\right) \cdot \mathcal{L}_{T_{j}^{\sigma}}\left(p_{j}\right)
\end{aligned}
$$

if $\delta>0$ is small. Setting now

$$
\widetilde{T}_{j}^{(\sigma)}:=\widehat{T}_{j}^{\sigma}+G_{v_{j}^{\sigma}},
$$

where $G_{v_{j}^{\sigma}}$ is the current integration over the graph of $v_{j}^{\sigma}$, the above construction and the boundary condition (5.13) yield that $\widetilde{T}_{j}^{(\sigma)}$ has no boundary in $\operatorname{int}\left(B_{j}\right) \times \mathcal{Y}$, so that $\widetilde{T}_{j}^{(\sigma)}$ belongs to cart ${ }^{1,1}\left(\operatorname{int}\left(B_{j}\right) \times \mathcal{Y}\right)$. Moreover, by (5.14) and (5.15), on account of the property vi) above, we obtain that

$$
\begin{aligned}
\mathcal{E}_{1,1}\left(\widetilde{T}_{j}^{(\sigma)}, \operatorname{int}\left(B_{j}\right) \times \mathcal{Y}\right) \leq & \mathcal{E}_{1,1}\left(T_{j}^{\sigma}, B_{R}^{n} \times \mathcal{Y}\right) \\
& +c \sigma r^{n-1}+c \sigma \mu_{J c, T_{j}^{\sigma}}\left(B_{r}^{n}\right) .
\end{aligned}
$$

We finally notice that $\widetilde{T}_{j}^{(\sigma)}$ agrees with $T_{j}^{\sigma}$ outside $\Omega_{\delta} \times \mathcal{Y}$.

Step 3: Flat distance. We now show that for $\delta$ small enough

$$
\mathbf{F}\left(\widetilde { T } _ { j } ^ { ( \sigma ) } \left\llcornerB_{R}^{n} \times \mathcal{Y}-T_{j}^{\sigma}\left\llcorner B_{R}^{n} \times \mathcal{Y}\right) \leq c \cdot \sigma \cdot R^{n-1} .\right.\right.
$$

In fact, by the property vii) above the blow-up current

$$
\widetilde{S}_{j}(\omega):=\lim _{\lambda \rightarrow 0^{+}} \eta_{0, \lambda \#} T_{j}^{\sigma}(\omega), \quad \omega \in \mathcal{Z}^{n, 1}\left(B_{R}^{n} \times \mathcal{Y}\right)
$$

is well-defined, and by property vi) it satisfies

$$
\widetilde{S}_{j}=\llbracket B_{R}^{+} \rrbracket \times \delta_{z^{+}}+\llbracket B_{R}^{-} \rrbracket \times \delta_{z^{-}}+\llbracket D_{r} \rrbracket \times \Gamma_{j},
$$


where $\partial \Gamma_{j}=\delta_{z^{+}}-\delta_{z^{-}}$. On the other hand, (5.7) yields that

$$
\mathbf{F}\left(\widetilde { S } _ { j } \left\llcornerB_{R}^{n} \times \mathcal{Y}-T_{j}^{\sigma}\left\llcorner B_{R}^{n} \times \mathcal{Y}\right) \leq c \cdot \sigma \cdot R^{n-1} .\right.\right.
$$

Also, by the definition of $v_{j}^{\sigma}$ we infer that for $\delta>0$ small

$$
\mathbf{F}\left(\widetilde { S } _ { j } \left\llcorner\widetilde{\Omega}_{\delta} \times \mathcal{Y}-G_{v_{j}^{\sigma}}\left\llcorner\widetilde{\Omega}_{\delta} \times \mathcal{Y}\right) \leq c \cdot \sigma \cdot r^{n-1} .\right.\right.
$$

Moreover, the $B V$-energy of $\widetilde{T}_{j}^{(\sigma)}$ on $\left(\Omega_{\delta} \backslash \widetilde{\Omega}_{\delta}\right) \times \mathcal{Y}$ is small if $\delta$ is small, whereas $\widetilde{T}_{j}^{(\sigma)}$ agrees with $T_{j}^{\sigma}$ outside $\Omega_{\delta} \times \mathcal{Y}$. By (5.18) we then obtain

$$
\mathbf{F}\left(\widetilde { S } _ { j } \left\llcorner\left(B_{R}^{n} \backslash \widetilde{\Omega}_{\delta}\right) \times \mathcal{Y}-\widetilde{T}_{j}^{(\sigma)}\left\llcorner\left(B_{R}^{n} \backslash \widetilde{\Omega}_{\delta}\right) \times \mathcal{Y}\right) \leq c \cdot \sigma \cdot R^{n-1}\right.\right.
$$

and finally (5.17), as $r \in(R / 2, R)$.

Step 4: Approximation on the whole domain. Setting now

$$
T_{j}^{(\sigma)}:=\left(\psi_{\sigma}^{-1} \bowtie \operatorname{Id}_{\mathbb{R}^{N}}\right)_{\#}\left(\widetilde{T}_{j}^{(\sigma)}\left\llcorner\operatorname{int}\left(B_{j}\right) \times \mathcal{Y}\right),\right.
$$

by (5.16), since $r=\rho_{j} \in\left(r_{j} / 2, r_{j}\right)$, we infer that for every $j$

$$
\mathcal{E}_{1,1}\left(T_{j}^{(\sigma)}, \operatorname{int}\left(B_{j}\right) \times \mathcal{Y}\right) \leq \int_{B_{j}}\left|\nabla u_{T}\right| d x+(1+c \sigma) \mu_{J c, T}\left(B_{j}\right)+c \sigma r_{j}^{n-1},
$$

whereas by (5.17), since $R=r_{j}$, we obtain that

$$
\mathbf{F}\left(T _ { j } ^ { ( \sigma ) } \left\llcorner\operatorname{int}\left(B_{j}\right) \times \mathcal{Y}-T\left\llcorner\operatorname{int}\left(B_{j}\right) \times \mathcal{Y}\right) \leq c \cdot \sigma \cdot r_{j}{ }^{n-1} .\right.\right.
$$

Let now $T^{\sigma} \in \operatorname{cart}^{1,1}\left(B^{n} \times \mathcal{Y}\right)$ be given by

$$
T^{\sigma}:=\sum_{j=1}^{\infty} T_{j}^{(\sigma)}+T\left\llcorner\left(B^{n} \backslash \bigcup_{j=1}^{\infty} \operatorname{int}\left(B_{j}\right)\right) \times \mathcal{Y} .\right.
$$

By (5.19) and (5.5) we obtain that

$$
\mathcal{E}_{1,1}\left(T^{\sigma}, B^{n} \times \mathcal{Y}\right) \leq \int_{B^{n}}\left|\nabla u_{T}\right| d x+(1+c \sigma) \mu_{J_{c}, T}\left(B^{n}\right)+c \sigma \mathcal{H}^{n-1}(J),
$$

so that if $\sigma=\sigma\left(\varepsilon, k, J, \mu_{J c, T}\right)>0$ is small, we have

$$
\mathcal{E}_{1,1}\left(T^{\sigma}, B^{n} \times \mathcal{Y}\right) \leq \mathcal{E}_{1,1}\left(T, B^{n} \times \mathcal{Y}\right)+\varepsilon^{k} .
$$


Moreover, by (5.4) and (5.6), taking $\sigma$ small, the above construction yields that

$$
\begin{aligned}
\mu_{J c, T^{\sigma}}\left(B^{n}\right) & \leq c \sum_{j=1}^{\infty} \mu_{J c, T}\left(B_{j} \backslash C_{j}\right)+\mu_{J c, T}\left(B^{n} \backslash J\right) \\
& \leq c \sigma \mu_{J c, T}\left(B^{n}\right)+\frac{1}{4} \mu_{J c, T}\left(B^{n}\right)<\frac{1}{2} \cdot \mu_{J c, T}\left(B^{n}\right) .
\end{aligned}
$$

Finally, by (5.20) we have

$$
\begin{aligned}
\mathbf{F}\left(T^{\sigma}-T\right) & \leq \sum_{j=1}^{\infty} \mathbf{F}\left(T _ { j } ^ { ( \sigma ) } \left\llcorner\operatorname{int}\left(B_{j}\right) \times \mathcal{Y}-T\left\llcorner\operatorname{int}\left(B_{j}\right) \times \mathcal{Y}\right)\right.\right. \\
& \leq c \cdot \sigma \sum_{j=1}^{\infty} r_{j}^{n-1}<\varepsilon^{k}
\end{aligned}
$$

if $\sigma=\sigma(\varepsilon, k)>0$ is small. Since $D u_{T^{\sigma}}$ has no Cantor part, the proof is complete.

\section{The total variation of $B V$-functions}

Extending the classical notion of total variation of vector-valued maps, to every map $u \in B V\left(B^{n}, \mathcal{Y}\right)$ we associate in a natural way its total variation, essentially in the sense of Jordan, given for every Borel set $B \subset B^{n}$ by

$$
\mathcal{E}_{T V}(u, B):=\int_{B}|\nabla u(x)| d x+\left|D^{C} u\right|(B)+\int_{J_{u} \cap B} \mathcal{H}^{1}\left(l_{x}\right) d \mathcal{H}^{n-1}(x) .
$$

Here, for any $x \in J_{u}$, we let $\mathcal{H}^{1}\left(l_{x}\right)$ denote the length of a geodesic arc $l_{x}$ in $\mathcal{Y}$ with initial and final points $u^{-}(x)$ and $u^{+}(x)$. Moreover we set

$$
\mathcal{E}_{T V}(u):=\mathcal{E}_{T V}\left(u, B^{n}\right) .
$$

Note that if $u$ is smooth, at least in $W^{1,1}\left(B^{n}, \mathcal{Y}\right)$, then

$$
\mathcal{E}_{T V}(u, B)=\mathcal{E}_{1,1}(u, B):=\int_{B}|D u| d x .
$$

Moreover, clearly for every $u \in B V\left(B^{n}, \mathcal{Y}\right)$ we have

$$
|D u|(B) \leq \mathcal{E}_{T V}(u, B) .
$$

Lower semicontinuity. In a way similar to Theorems 1.7 and 2.12, it is not difficult to prove in any dimension $n$ the following: 
Proposition 6.1. Let $u \in B V\left(B^{n}, \mathcal{Y}\right)$. For every sequence of smooth maps $\left\{u_{k}\right\} \subset$ $C^{1}\left(B^{n}, \mathcal{Y}\right)$ such that $u_{k} \rightarrow u$ weakly in the $B V$-sense, we have

$$
\mathcal{E}_{T V}(u) \leq \liminf _{k \rightarrow \infty} \mathcal{E}_{T V}\left(u_{k}\right)
$$

The previous definition is motivated by the 1-dimensional case, $n=1$. In fact, similarly to Theorem 1.8 , we can prove the following:

Theorem 6.2. For every $u \in B V\left(B^{1}, \mathcal{Y}\right)$ there exists a sequence of smooth maps $\left\{u_{k}\right\} \subset C^{\infty}\left(B^{1}, \mathcal{Y}\right)$ such that $u_{k} \rightarrow u$ weakly in the $B V$-sense and $\mathcal{E}_{T V}\left(u_{k}\right) \rightarrow$ $\mathcal{E}_{T V}(u)$ as $k \rightarrow \infty$.

Density results for Sobolev maps. If $n \geq 2$, we denote by $R_{1}^{\infty}\left(B^{n}, \mathcal{Y}\right)$ the set of all the maps $u \in W^{1,1}\left(B^{n}, \mathcal{Y}\right)$ which are smooth except on a singular set $\Sigma(u)$ of the type

$$
\Sigma(u)=\bigcup_{i=1}^{r} \Sigma_{i}, \quad r \in \mathbb{N},
$$

where $\Sigma_{i}$ is a smooth $(n-2)$-dimensional subset of $B^{n}$ with smooth boundary, if $n \geq 3$, and $\Sigma_{i}$ is a point if $n=2$. The following density results appear in [5].

Theorem 6.3. The class $R_{1}^{\infty}\left(B^{n}, \mathcal{Y}\right)$ is strongly dense in $W^{1,1}\left(B^{n}, \mathcal{Y}\right)$.

Theorem 6.4. The class $C^{1}\left(B^{n}, \mathcal{Y}\right)$ is dense in $R_{1}^{\infty}\left(B^{n}, \mathcal{Y}\right)$ in the strong $W^{1,1}$ topology if and only if $\pi_{1}(\mathcal{Y})=0$.

Using arguments from the proof of Theorem 2.13, it is not difficult to extend Theorem 6.3 to maps in $B V\left(B^{n}, \mathcal{Y}\right)$, by proving:

Theorem 6.5. For every $u \in B V\left(B^{n}, \mathcal{Y}\right)$ there exists a sequence of maps $\left\{u_{k}\right\} \subset$ $R_{1}^{\infty}\left(B^{n}, \mathcal{Y}\right)$ such that $u_{k} \rightarrow u$ as $k \rightarrow \infty$ weakly in the $B V$-sense and

$$
\lim _{k \rightarrow \infty} \int_{B^{n}}\left|D u_{k}\right| d x=\mathcal{E}_{T V}\left(u, B^{n}\right) .
$$

As a consequence, by using Theorem 6.4 we immediately obtain:

Corollary 6.6. Suppose that $\pi_{1}(\mathcal{Y})=0$. For every $u \in B V\left(B^{n}, \mathcal{Y}\right)$ there exists a sequence of smooth maps $\left\{u_{k}\right\} \subset C^{1}\left(B^{n}, \mathcal{Y}\right)$ such that $u_{k} \rightarrow u$ as $k \rightarrow \infty$ weakly in the $B V$-sense and (6.2) holds true.

Currents carried by $\boldsymbol{B} \boldsymbol{V}$-functions. Following Section 2, the structure of a function $u$ in $B V\left(B^{n}, \mathcal{Y}\right)$ suggests to associate to $u$ a suitable current $G=T_{u} \in$ $B V$-graph $\left(B^{n} \times \mathcal{Y}\right)$, see Definition 2.1, where the function $u\left(T_{u}\right) \in B V\left(B^{n}, \mathcal{Y}\right)$ is equal to $u$ and the $\gamma_{x}$ 's in the definition of the jump part $G_{u}^{J}$ agree for every $x \in J_{u}$ with an oriented geodesic arc $l_{x}$ in $\mathcal{Y}$ with initial and final points respectively given by $u^{-}(x)$ and $u^{+}(x)$, so that $\partial \llbracket l_{x} \rrbracket=\delta_{u^{+}(x)}-\delta_{u^{-}(x)}$. We notice 
that the definition of $T_{u}$ depends on the choice of the geodesics $l_{x}$. In particular, if $u \in W^{1,1}\left(B^{n}, \mathcal{Y}\right)$, clearly $T_{u}=T_{u}^{a}$ and hence $T_{u}$ agrees with the current $G_{u}$ integration of forms in $\mathcal{D}^{n, 1}\left(B^{n} \times \mathcal{Y}\right)$ over the rectifiable graph of $u$, see (2.1). Now, Definition 2.5 yields that the parametric variational integral $\mathcal{F}_{1,1}$ associated to the total variation integral is such that for every Borel set $B \subset B^{n}$

$$
\mathcal{F}_{1,1}\left(T_{u}, B \times \mathcal{Y}\right)=\mathcal{E}_{T V}(u, B) \quad \forall u \in B V\left(B^{n}, \mathcal{Y}\right)
$$

Moreover, arguing as in the proof of Theorem 2.13, we readily extend Theorems 6.2 and 6.5 by proving in any dimension $n \geq 2$

Theorem 6.7. For every $u \in B V\left(B^{n}, \mathcal{Y}\right)$ we find the existence of a sequence of maps $\left\{u_{k}\right\} \subset R_{1}^{\infty}\left(B^{n}, \mathcal{Y}\right)$ such that $u_{k} \rightarrow u$ weakly in the $B V$-sense, $G_{u_{k}} \rightarrow T_{u}$ weakly in $\mathcal{Z}_{n, 1}\left(B^{n} \times \mathcal{Y}\right)$ and

$$
\lim _{k \rightarrow \infty} \int_{B^{n}}\left|D u_{k}\right| d x=\mathcal{E}_{T V}\left(u, B^{n}\right)
$$

Remark 6.8. If $n \geq 2$ in general the current $T_{u}$ has a non zero boundary in $B^{n} \times$ $\mathcal{Y}$, compare Remark 2.2. However, as shown by Proposition 6.9 below, $\partial T_{u}$ is null on every $(n-1)$-form $\widetilde{\omega}$ in $B^{n} \times \mathcal{Y}$ which has no "vertical" differentials. To this purpose, following Proposition 2.3, any smooth $(n-1)$-form $\widetilde{\omega} \in \mathcal{D}^{n-1}\left(B^{n} \times \mathcal{Y}\right)$ with no vertical differentials can be written as $\widetilde{\omega}:=\omega_{\varphi} \wedge \eta$ for some $\eta \in C_{0}^{\infty}(\mathcal{Y})$ and $\varphi=\left(\varphi^{1}, \ldots, \varphi^{n}\right) \in C_{0}^{\infty}\left(B^{n}, \mathbb{R}^{n}\right)$, where $\omega_{\varphi}$ is given by (2.5). Since $d_{x} \widetilde{\omega}=$ $d \omega_{\varphi} \wedge \eta=\operatorname{div} \varphi(x) \eta(y) d x$, by Definition 2.1 we have

$$
\begin{aligned}
\partial_{x} T_{u}(\widetilde{\omega}) & :=T_{u}\left(d_{x} \widetilde{\omega}\right)=T_{u}(\operatorname{div} \varphi(x) \eta(y) d x) \\
& =\int_{B^{n}} \operatorname{div} \varphi(x) \cdot \eta(u(x)) d x .
\end{aligned}
$$

We now show that $\partial_{y} T_{u}(\widetilde{\omega})=-\partial_{x} T_{u}(\widetilde{\omega})$, which yields the assertion.

Proposition 6.9. We have

$$
\begin{aligned}
\partial_{y} T_{u}\left(\omega_{\varphi} \wedge \eta\right): & =T_{u}\left(d_{y}\left(\omega_{\varphi} \wedge \eta\right)\right) \\
& =-\int_{B^{n}} \operatorname{div} \varphi(x) \cdot \eta(u(x)) d x=:\langle D(\eta \circ u), \varphi\rangle .
\end{aligned}
$$

Proof. Since

$$
\begin{aligned}
d_{y}\left(\omega_{\varphi} \wedge \eta\right) & =(-1)^{n-1} \omega_{\varphi} \wedge d_{y} \eta \\
& =\sum_{j=1}^{N} \sum_{i=1}^{n}(-1)^{n-i} \varphi^{i}(x) \frac{\partial \eta}{\partial y^{j}}(y) \widehat{d x^{i}} \wedge d y^{j}
\end{aligned}
$$


taking $\phi_{i}^{j}=\varphi^{i} \eta, y_{j}$ in (2.2), by the definition of $T_{u}$ we infer

$$
\begin{aligned}
(-1)^{n-1} T_{u}\left(\omega_{\varphi} \wedge d_{y} \eta\right)= & \sum_{j=1}^{N} \int_{B^{n}} \frac{\partial \eta}{\partial y^{j}}(u(x))\left\langle\nabla u^{j}(x), \varphi(x)\right\rangle d x \\
& +\sum_{j=1}^{N} \int_{B^{n}} \frac{\partial \eta}{\partial y^{j}}(u(x)) \varphi(x) d D^{C} u^{j} \\
& +\int_{J_{u}}\left(\eta\left(u^{+}(x)\right)-\eta\left(u^{-}(x)\right)\langle\varphi(x), v(x)\rangle d \mathcal{H}^{n-1} .\right.
\end{aligned}
$$

Therefore, by the chain rule formula for the distributional derivative of $\eta \circ u$, compare [2], we obtain the assertion, as

$$
\left.T_{u}\left(d_{y}\left(\omega_{\varphi} \wedge \eta\right)\right)=(-1)^{n-1} T_{u}\left(\omega_{\varphi} \wedge d_{y} \eta\right)\right)=\langle D(\eta \circ u), \varphi\rangle .
$$

Remark 6.10. If $G$ is any current in $B V-\operatorname{graph}\left(B^{n} \times \mathcal{Y}\right)$ with corresponding function $u(G) \in B V\left(B^{n}, \mathcal{Y}\right)$ equal to $u$, see Definition 2.1, arguing as in Proposition 6.9 we obtain again that

$$
\partial_{x} G\left(\omega_{\varphi} \wedge \eta\right)=-\partial_{y} G\left(\omega_{\varphi} \wedge \eta\right)=\int_{B^{n}} \operatorname{div} \varphi(x) \cdot \eta(u(x)) d x
$$

Example 6.11. Of course, compare Section 2, every Cartesian current $T$ in cart $^{1,1}\left(B^{n} \times \mathcal{Y}\right)$ may be decomposed as

$$
T=T_{u}+S_{T} \quad \text { on } \quad \mathcal{Z}^{n, 1}\left(B^{n} \times \mathcal{Y}\right),
$$

where $u=u_{T} \in B V\left(B^{n}, \mathcal{Y}\right)$ is the $B V$-function corresponding to $T$ and $T_{u} \in$ $B V-\operatorname{graph}\left(B^{n} \times \mathcal{Y}\right)$ is defined as above, by means of geodesic arcs connecting $u^{-}(x)$ and $u^{+}(x)$ at the points $x$ in the jump set $J_{u}$. However, even in dimension $n=1$ and in the particular case $\mathcal{Y}=S^{1}$, the unit sphere, in general it may happen that the $B V$-energy of $T$ cannot be recovered by the sum of the $B V$-energies of its component $T_{u}$ and $S_{T}$ in (6.3). If $\mathcal{Y}=S^{1}$, in fact, we have $S_{T \text {, sing }}=0$, i.e., the equivalence classes of elements in $\operatorname{cart}^{1,1}\left(B^{n} \times S^{1}\right)$ have a unique representative, and the energies $\mathcal{E}_{1,1}(T)$ and $\mathcal{F}_{1,1}(T)$ are equal, i.e., no gap phenomenon occurs. Consider the current $T^{\theta} \in \operatorname{cart}^{1,1}\left(B^{1} \times S^{1}\right)$ given by

$$
T^{\theta}:=\llbracket(-1,0) \rrbracket \times \delta_{P_{0}}+\llbracket(0,1) \rrbracket \times \delta_{P_{\theta}}+\delta_{0} \times \gamma_{\theta}, \quad \theta \in[0,2 \pi],
$$

where $P_{\theta}=(\cos \theta, \sin \theta)$ and $\gamma_{\theta}$ is the simple arc in $S^{1}$ connecting the points $P_{0}$ and $P_{\theta}$ in the counterclockwise sense. If $\pi<\theta<2 \pi$ we clearly have

$$
T_{u}=\llbracket(-1,0) \rrbracket \times \delta_{P_{0}}+\llbracket(0,1) \rrbracket \times \delta_{P_{\theta}}+\delta_{0} \times \tilde{\gamma}_{\theta},
$$


where $\widetilde{\gamma}_{\theta}$ is the simple arc in $S^{1}$ connecting the points $P_{0}$ and $P_{\theta}$ in the clockwise sense, so that we may decompose $T^{\theta}$ as in (6.3) with $S_{T}=\delta_{0} \times \llbracket\left[S^{1} \rrbracket\right.$. Since

$$
\mathcal{F}_{1,1}\left(T_{u}\right)=\mathcal{H}^{1}\left(\tilde{\gamma}_{\theta}\right)=2 \pi-\theta, \quad \mathcal{F}_{1,1}\left(S_{T}\right)=2 \pi,
$$

we infer that the sum of the energies $\mathcal{F}_{1,1}\left(T_{u}\right)+\mathcal{F}_{1,1}\left(S_{T}\right)$ is greater than the energy of $T^{\theta}$, as clearly

$$
\mathcal{E}_{1,1}\left(T^{\theta}\right)=\mathcal{F}_{1,1}\left(T^{\theta}\right)=\mathcal{H}^{1}\left(\gamma_{\theta}\right)=\theta .
$$

\section{The relaxed $B V$-energy of functions}

In this section we analyze the lower semicontinuous envelope of the total variation, defined for every function $u \in B V\left(B^{n}, \mathcal{Y}\right)$ by

$$
\begin{gathered}
\widetilde{\mathcal{E}_{T V}}(u):=\inf \left\{\liminf _{k \rightarrow \infty} \int_{B^{n}}\left|D u_{k}\right| d x \mid\left\{u_{k}\right\} \subset C^{1}\left(B^{n}, \mathcal{Y}\right),\right. \\
\left.u_{k} \rightarrow u \quad \text { weakly in the } B V \text {-sense }\right\} .
\end{gathered}
$$

Remark 7.1. Of course one may equivalently require that $u_{k} \rightarrow u$ strongly in $L^{1}\left(B^{n}, \mathbb{R}^{N}\right)$.

We first recall the following facts.

Definition 7.2. For every $k=2, \ldots, n$ and $\Gamma \in \mathcal{D}_{n-k}\left(B^{n}\right)$, we denote by

$$
m_{i, B^{n}}(\Gamma):=\inf \left\{\mathbf{M}(L) \mid L \in \mathcal{R}_{n-k+1}\left(B^{n}\right), \quad(\partial L)\left\llcorner B^{n}=\Gamma\right\}\right.
$$

the integral mass of $\Gamma$ and by

$$
m_{r, B^{n}}(\Gamma):=\inf \left\{\mathbf{M}(D) \mid D \in \mathcal{D}_{n-k+1}\left(B^{n}\right),(\partial D)\left\llcorner B^{n}=\Gamma\right\}\right.
$$

the real mass of $\Gamma$. Moreover, in case $m_{i, B^{n}}(\Gamma)<\infty$, we say that an integer multiplicity rectifiable current $L \in \mathcal{R}_{n-k+1}\left(B^{n}\right)$ is an integral minimal connection of $\Gamma$ if $(\partial L)\left\llcorner B^{n}=\Gamma\right.$ and $\mathbf{M}(L)=m_{i, B^{n}}(\Gamma)$.

We also recall that by Federer's theorem [10], and by Hardt-Pitts' result [18], respectively, in the cases $k=n$ and $k=2$ we have that

$$
m_{i, B^{n}}(\Gamma)=m_{r, B^{n}}(\Gamma) .
$$

Vertical homology classes. Let $u \in W^{1,1}\left(B^{n}, \mathcal{Y}\right)$ and let $G_{u}$ be the current integration of forms in $\mathcal{D}^{n, 1}\left(B^{n} \times \mathcal{Y}\right)$ over the rectifiable graph of $u$, see (2.1). We have that $\partial G_{u}(\omega)=0$ if $\omega \in \mathcal{D}^{n-1,1}\left(B^{n} \times \mathcal{Y}\right)$ with $\omega^{(1)}=0$ or $d_{y} \omega=0$. Setting

$$
\mathcal{B}^{p, 1}\left(B^{n} \times \mathcal{Y}\right):=\left\{\omega \in \mathcal{D}^{p, 1}\left(B^{n} \times \mathcal{Y}\right) \mid \exists \eta \in \mathcal{D}^{p-1,0}\left(B^{n} \times \mathcal{Y}\right): \omega^{(1)}=d_{y} \eta\right\}
$$


and

$$
\mathcal{H}^{p, 1}\left(B^{n} \times \mathcal{Y}\right):=\frac{\mathcal{Z}^{p, 1}\left(B^{n} \times \mathcal{Y}\right)}{\mathcal{B}^{p, 1}\left(B^{n} \times \mathcal{Y}\right)},
$$

then $\partial G_{u}=0$ on $\mathcal{B}^{n-1,1}\left(B^{n} \times \mathcal{Y}\right)$ and $\partial_{y} \partial G_{u}=0$, whence $\partial G_{u}(\omega)$ depends only on the cohomology class of $\omega \in \mathcal{Z}^{n-1,1}\left(B^{n} \times \mathcal{Y}\right)$. As a consequence $\partial G_{u}$ induces a functional $\left(\partial G_{u}\right)_{\star}$ on $\mathcal{H}^{n-1,1}\left(B^{n} \times \mathcal{Y}\right)$ given by

$$
\left(\partial G_{u}\right)_{\star}\left(\omega+\mathcal{B}^{n-1,1}\right):=\partial G_{u}\left(\omega+\mathcal{B}^{n-1,1}\right)=\partial G_{u}(\omega), \quad \omega \in \mathcal{Z}^{n-1,1},
$$

compare [14], Vol. II, Section 5.4.1. Therefore, since

$$
\mathcal{H}^{p, 1}\left(B^{n} \times \mathcal{Y}\right) \simeq \mathcal{D}^{p-1}\left(B^{n}\right) \otimes H_{d R}^{1}(\mathcal{Y})
$$

the homology map $\left(\partial G_{u}\right)_{\star}$ is uniquely represented as an element of $\mathcal{D}_{n-2}\left(B^{n}\right.$; $\left.H_{1}(\mathcal{Y} ; \mathbb{R})\right)$. More explicitly, if $\phi \in \mathcal{D}^{n-2}\left(B^{n}\right)$, we have $\left[\left(\partial G_{u}\right)_{\star}(\phi)\right] \in H_{1}(\mathcal{Y} ; \mathbb{R})$ and for $s=1, \ldots, \bar{s}$

$$
\left\langle\left(\partial G_{u}\right)_{\star}(\phi),\left[\omega^{s}\right]\right\rangle=\partial G_{u}\left(\pi^{\#} \phi \wedge \widehat{\pi}^{\#} \omega^{s}\right),
$$

$\langle$,$\rangle denoting the de Rham duality between H_{1}(\mathcal{Y} ; \mathbb{R})$ and $H_{d R}^{1}(\mathcal{Y})$ : in general $\left(\partial G_{u}\right)_{\star}$ is non-trivial.

Singularities of Sobolev maps. Following [14], Vol. II, Section 5.4.2, we now set

$$
\mathbb{P}(u):=\left(\partial G_{u}\right)_{\star} \in \mathcal{D}_{n-2}\left(B^{n} ; H_{1}(\mathcal{Y} ; \mathbb{R})\right)
$$

and for each $\omega \in[\omega] \in H_{d R}^{1}(\mathcal{Y})$ we define the current $\mathbb{P}(u ; \omega):=-\pi_{\#}\left(\left(\partial G_{u}\right)\left\llcorner\widehat{\pi}^{\#} \omega\right) \in\right.$ $\mathcal{D}_{n-2}\left(B^{n}\right)$, so that

$$
\mathbb{P}(u ; \omega)(\phi)=-\partial G_{u}\left(\widehat{\pi}^{\#} \omega \wedge \pi^{\#} \phi\right)=G_{u}\left(\widehat{\pi}^{\#} \omega \wedge \pi^{\#} d \phi\right)=\int_{B^{n}} u^{\#} \omega \wedge d \phi
$$

for every $\phi \in \mathcal{D}^{n-2}\left(B^{n}\right)$. We also define for every $\omega \in \mathcal{Z}^{1}(\mathcal{Y})$ the current $\mathbb{D}(u ; \omega):=\pi_{\#}\left(G_{u}\left\llcorner\widehat{\pi}^{\#} \omega\right) \in \mathcal{D}_{n-1}\left(B^{n}\right)\right.$, so that

$$
\mathbb{D}(u ; \omega)(\gamma)=G_{u}\left(\widehat{\pi}^{\#} \omega \wedge \pi^{\#} \gamma\right)=\int_{B^{n}} u^{\#} \omega \wedge \gamma \quad \forall \gamma \in \mathcal{D}^{n-1}\left(B^{n}\right) .
$$

The following facts hold:

(i) for $s=1, \ldots, \bar{s}$

$$
\mathbb{P}\left(u ; \omega^{s}\right)(\phi)=\left\langle\mathbb{P}(u)(\phi),\left[\omega^{s}\right]\right\rangle,
$$

i.e., $\mathbb{P}\left(u ; \omega^{s}\right)$ does not depend on the representative in the cohomology class $\left[\omega^{s}\right]$ 
(ii) $\partial \mathbb{P}(u)=0$ and $\mathbb{P}(u)=\sum_{s=1}^{\bar{s}} \mathbb{P}\left(u ; \omega^{s}\right) \otimes\left[\gamma_{s}\right]$, hence it does not depend on the choice of $\gamma_{1}, \ldots, \gamma_{\bar{s}}$;

(iii) $\partial \mathbb{D}(u ; \omega)(\phi)=\langle\mathbb{P}(u)(\phi),[\omega]\rangle$ and hence $\partial \mathbb{D}\left(u ; \widetilde{\omega}^{s}\right)\left\llcorner B^{n}=\mathbb{P}\left(u ; \widetilde{\omega}^{s}\right)\right.$ for each representative $\widetilde{\omega}^{s}$ in $\left[\omega^{s}\right]$.

We can therefore set

$$
\mathbb{D}_{s}(u):=\mathbb{D}\left(u ; \omega^{s}\right), \quad \mathbb{P}_{s}(u):=\mathbb{P}\left(u ; \omega^{s}\right)=\partial \mathbb{D}_{s}(u)\left\llcorner B^{n}, \quad s=1, \ldots, \bar{s} .\right.
$$

Notice that if $T \in \operatorname{cart}^{1,1}\left(B^{n} \times \mathcal{Y}\right)$ satisfies

$$
T=G_{u}+S_{T}, \quad S_{T}=\sum_{s=1}^{\bar{s}} \mathbb{L}_{s}(T) \times \gamma_{s} \quad \text { on } \quad \mathcal{Z}^{n, 1}\left(B^{n} \times \mathcal{Y}\right),
$$

where $u=u_{T} \in W^{1,1}\left(B^{n}, \mathcal{Y}\right)$ and $\mathbb{L}_{s}(T) \in \mathcal{R}_{n-1}\left(B^{n}\right)$, since

$$
\begin{aligned}
(-1)^{n-2} \partial G_{u}\left(\widehat{\pi}^{\#} \omega^{s} \wedge \pi^{\#} \phi\right) & =\partial G_{u}\left(\pi^{\#} \phi \wedge \widehat{\pi}^{\#} \omega^{s}\right)=-\partial S_{T}\left(\pi^{\#} \phi \wedge \widehat{\pi}^{\#} \omega^{s}\right) \\
& =-\partial \mathbb{L}_{s}(T)(\phi),
\end{aligned}
$$

we infer that

$$
\mathbb{P}_{s}(u)=(-1)^{n} \partial \mathbb{L}_{s}(T)\left\llcorner B^{n} \quad \forall s=1, \ldots, \bar{s} .\right.
$$

Finally, we clearly have $\mathbb{P}(u)=0$ if $u$ is smooth, say Lipschitz, or at least in $W^{1,2}\left(B^{n}, \mathcal{Y}\right)$.

Results. In the sequel we shall assume that the first homotopy group $\pi_{1}(\mathcal{Y})$ is commutative. Moreover, we denote by

$$
\mathcal{T}_{u}:=\left\{T \in \operatorname{cart}^{1,1}\left(B^{n}, \mathcal{Y}\right) \mid u_{T}=u\right\}
$$

the class of Cartesian currents $T$ in cart $^{1,1}\left(B^{n} \times \mathcal{Y}\right)$ such that the underlying $B V$ function $u_{T}$ is equal to $u$, compare Definition 2.11 and Remark 2.7. We first prove

Theorem 7.3. For every $u \in B V\left(B^{n}, \mathcal{Y}\right)$ we have $\widetilde{\mathcal{E}_{T V}}(u)<\infty$.

From the results of the previous sections we then obtain the following representation result.

Theorem 7.4. For any $u \in B V\left(B^{n}, \mathcal{Y}\right)$ we have

$$
\begin{aligned}
\widetilde{\mathcal{E}_{T V}}(u)= & \inf \left\{\mathcal{E}_{1,1}(T) \mid T \in \mathcal{T}_{u}\right\} \\
= & \int_{B^{n}}|\nabla u(x)| d x+\left|D^{C} u\right|\left(B^{n}\right) \\
& +\inf \left\{\int_{J_{c}(T)} \mathcal{L}_{T}(x) d \mathcal{H}^{n-1}(x) \mid T \in \mathcal{T}_{u}\right\},
\end{aligned}
$$

where $\mathcal{T}_{u}, J_{c}(T)$, and $\mathcal{L}_{T}(x)$ are given by (7.4), (2.12), and Definition 2.9, respectively. 
Proof of Theorem 7.3. We observe that it suffices to show that the class $\mathcal{T}_{u}$ is non-empty, see (7.4). In this case, in fact, if $T \in \mathcal{T}_{u}$, by Theorem 2.13 we find a smooth sequence $\left\{u_{k}\right\} \subset C^{1}\left(B^{n}, \mathcal{Y}\right)$ such that $G_{u_{k}} \rightarrow T$ weakly in $\mathcal{Z}_{n, 1}\left(B^{n} \times \mathcal{Y}\right)$ and $\left\|D u_{k}\right\|_{L^{1}} \rightarrow \mathcal{E}_{1,1}(T)$ as $k \rightarrow \infty$; this yields also that $u_{k} \rightarrow u_{T}$ weakly in the $B V$-sense, where $u_{T}=u$, whence $\widetilde{\mathcal{E}_{T V}}(u)<\infty$.

Now let us prove that $\mathcal{T}_{u}$ is non-empty. We first notice that, since $\mathcal{Y}$ is smooth and compact, there exists an absolute constant $C>0$, depending on $\mathcal{Y}$, such that

$$
\mathcal{E}_{T V}\left(u, B^{n}\right)<C|D u|\left(B^{n}\right)<\infty .
$$

Let $\left\{u_{k}\right\}$ be the approximating sequence given by Theorem 6.7. Since $u_{k} \in$ $R_{1}^{\infty}\left(B^{n}, \mathcal{Y}\right)$, the real mass of the singularities is bounded by the $L^{1}$-norm of $D u_{k}$. More precisely, there exists an absolute constant $C>0$ such that

$$
m_{r, B^{n}}\left(\mathbb{P}_{s}\left(u_{k}\right)\right) \leq C \int_{B^{n}}\left|D u_{k}\right| d x \quad \forall s=1, \ldots, \bar{s},
$$

see Definition 7.2. In fact, we have

$$
\begin{aligned}
\mathbf{M}\left(\mathbb{D}_{s}\left(u_{k}\right)\right) & =\sup \left\{\int_{B^{n}} \phi \wedge\left(u_{k}^{\#} \omega^{s}\right) \mid \phi \in \mathcal{D}^{n-1}\left(B^{n}\right),\|\phi\| \leq 1\right\} \\
& \leq C \int_{B^{n}}\left|D u_{k}\right| d x,
\end{aligned}
$$

see Proposition 7.6 below for the case $\mathcal{Y}=S^{1}$, so that the assertion follows from (7.2). Therefore, since by Hardt-Pitts' result (7.1) we have

$$
m_{i, B^{n}}\left(\mathbb{P}_{s}\left(u_{k}\right)\right)=m_{r, B^{n}}\left(\mathbb{P}_{s}\left(u_{k}\right)\right),
$$

we find for every $s$ an integer multiplicity rectifiable current $\mathbb{L}_{s}^{k} \in \mathcal{R}_{n-1}\left(B^{n}\right)$ such that

$$
\mathbb{P}_{s}\left(u_{k}\right)=(-1)^{n}\left(\partial \mathbb{L}_{s}^{k}\right)\left\llcorner B^{n} \quad \text { and } \quad \mathbf{M}\left(\mathbb{L}_{s}^{k}\right) \leq C \int_{B^{n}}\left|D u_{k}\right| d x,\right.
$$

compare (7.3). As a consequence, letting

$$
T_{k}:=G_{u_{k}}+\sum_{s=1}^{\bar{s}} \mathbb{L}_{s}^{k} \times \gamma_{s}
$$

we readily find that $T_{k} \in \mathcal{D}_{n, 1}\left(B^{n} \times \mathcal{Y}\right)$ has no interior boundary

$$
\partial T_{k}=0 \quad \text { on } \quad \mathcal{Z}^{n-1,1}\left(B^{n} \times \mathcal{Y}\right)
$$

and finite $B V$-energy

$$
\mathcal{E}_{1,1}\left(T_{k}\right) \leq \int_{B^{n}}\left|D u_{k}\right| d x+C(\mathcal{Y}) \sum_{s=1}^{\bar{s}} \mathbf{M}\left(\mathbb{L}_{s}^{k}\right) \cdot \mathbf{M}\left(\gamma_{s}\right)<\infty
$$


for some absolute constant $C(\mathcal{Y})>0$. In conclusion, by (7.6) we obtain a sequence $\left\{T_{k}\right\} \subset \operatorname{cart}^{1,1}\left(B^{n} \times \mathcal{Y}\right)$ with equibounded energies

$$
\sup _{k} \mathcal{E}_{1,1}\left(T_{k}\right) \leq \sup _{k} C \int_{B^{n}}\left|D u_{k}\right| d x \leq C \mathcal{E}_{T V}\left(u, B^{n}\right)<\infty,
$$

where $C>0$ is an absolute constant. Therefore, by compactness, Proposition 2.18, possibly passing to a subsequence we find that $T_{k} \rightarrow T$ weakly in $\mathcal{Z}_{n, 1}\left(B^{n} \times \mathcal{Y}\right)$ to some $T \in \operatorname{cart}^{1,1}\left(B^{n} \times \mathcal{Y}\right)$ satisfying

$$
\mathcal{E}_{1,1}(T) \leq \liminf _{k \rightarrow \infty} \mathcal{E}_{1,1}\left(T_{k}\right)<\infty
$$

by lower semicontinuity, Proposition 2.16. In particular, since $u_{k} \rightarrow u$ weakly in the $B V$-sense, we find that the underlying $B V$-function $u_{T}=u$ and hence that $T \in \mathcal{T}_{u}$.

Proof of Theorem 7.4. Let $\left\{u_{k}\right\} \subset C^{1}\left(B^{n}, \mathcal{Y}\right)$ be a sequence of smooth maps with equibounded energies, $\sup _{k}\left\|D u_{k}\right\|_{L^{1}}<\infty$, weakly converging to $u$ in the $B V$-sense, see Theorem 7.3. By compactness, Proposition 2.18 , possibly passing to a subsequence we find that $G_{u_{k}} \rightarrow T$ weakly in $\mathcal{Z}_{n, 1}\left(B^{n} \times \mathcal{Y}\right)$ to some $T \in$ cart $^{1,1}\left(B^{n} \times \mathcal{Y}\right)$ satisfying $u_{T}=$ u, i.e. $T \in \mathcal{T}_{u}$, see (7.4). Since by lower semicontinuity, Proposition 2.16,

$$
\mathcal{E}_{1,1}(T) \leq \liminf _{k \rightarrow \infty} \int_{B^{n}}\left|D u_{k}\right| d x,
$$

we readily conclude that

$$
\inf \left\{\mathcal{E}_{1,1}(T) \mid T \in \mathcal{T}_{u}\right\} \leq \widetilde{\mathcal{E}_{T V}}(u) .
$$

To prove the opposite inequality, by applying Theorem 2.13, for every $T \in \mathcal{T}_{u}$ we find a smooth sequence $\left\{u_{k}\right\} \subset C^{1}\left(B^{n}, \mathcal{Y}\right)$ such that $G_{u_{k}} \rightarrow T$ weakly in $\mathcal{Z}_{n, 1}\left(B^{n} \times \mathcal{Y}\right)$ and $\left\|D u_{k}\right\|_{L^{1}} \rightarrow \mathcal{E}_{1,1}(T)$ as $k \rightarrow \infty$. Since the weak convergence $G_{u_{k}} \rightarrow T$ yields the convergence $u_{k} \rightarrow u_{T}$ weakly in the $B V$-sense, and $u_{T}=u$, we find that $\widetilde{\mathcal{E}_{T V}}(u) \leq \mathcal{E}_{1,1}(T)$, which proves the first equality in (7.5). The second equality in (7.5) follows from the definition of $B V$-energy, Definition 2.10.

The above results simplify if we specify them to $u \in W^{1,1}\left(B^{n}, \mathcal{Y}\right)$ and/or $\mathcal{Y}=S^{1}$, recovering this way previous results, compare e.g. [13], [8], and [19].

The relaxed $\boldsymbol{W}^{\mathbf{1}, \mathbf{1}}$-energy. The relaxed energy of $u \in W^{1,1}\left(B^{n}, \mathcal{Y}\right)$ is of course given by

$\widetilde{\mathcal{E}_{1,1}}(u):=\inf \left\{\liminf _{k \rightarrow \infty} \int_{B^{n}}\left|D u_{k}\right| d x \mid\left\{u_{k}\right\} \subset C^{1}\left(B^{n}, \mathcal{Y}\right), u_{k} \rightarrow u\right.$ strongly in $\left.L^{1}\left(B^{n}, \mathbb{R}^{N}\right)\right\}$,

see Remark 7.1. In this case, Theorem 7.4 reads as: 
Corollary 7.5. For any $u \in W^{1,1}\left(B^{n}, \mathcal{Y}\right)$ we have $\widetilde{\mathcal{E}_{1,1}}(u)<\infty$. Every $T \in \mathcal{T}_{u}$ has the form

$$
T=G_{u}+\sum_{q \in H_{1}(\mathcal{Y})} \mathbb{L}_{q} \times C_{q} \quad \text { on } \quad \mathcal{Z}^{n, 1}\left(B^{n} \times \mathcal{Y}\right),
$$

where $\mathbb{L}_{q}=\tau\left(\mathcal{L}_{q}, 1, \overrightarrow{\mathcal{L}}_{q}\right)$ is an integer multiplicity rectifiable current in $\mathcal{R}_{n-1}\left(B^{n}\right)$ and $C_{q} \in \mathcal{Z}_{1}(\mathcal{Y})$ is an integral 1-cycle in the homology class $q$, and its $B V$-energy is given by

$$
\mathcal{E}_{1,1}(T)=\int_{B^{n}}|D u| d x+\sum_{q \in H_{1}(\mathcal{Y})} \int_{\mathcal{L}_{q}} \mathcal{L}_{T}(x) d \mathcal{H}^{n-1}(x)
$$

where, for $x \in \mathcal{L}_{q}$, we have $\mathcal{L}_{T}(x):=\inf \left\{\mathcal{L}(\gamma) \mid \gamma \in \Gamma_{q}(x)\right\}$ and

$$
\Gamma_{q}(x):=\left\{\gamma \in \operatorname{Lip}([0,1], \mathcal{Y}) \mid \gamma(0)=\gamma(1)=u(x), \quad \gamma_{\#} \llbracket(0,1) \rrbracket \in q\right\} .
$$

The relaxed energy is given by

$$
\widetilde{\mathcal{E}_{1,1}}(u)=\int_{B^{n}}|D u(x)| d x+\inf \left\{\sum_{q \in H_{1}(\mathcal{Y})} \int_{\mathcal{L}_{q}} \mathcal{L}_{T}(x) d \mathcal{H}^{n-1}(x) \mid T \in \mathcal{T}_{u}\right\} .
$$

The case $\mathcal{Y}=\mathcal{S}^{\mathbf{1}}$. Further simplification arises if we assume $\mathcal{Y}=S^{1}$. In this case, in fact, $S_{T, \text { sing }}=0$, i.e. the equivalence classes of elements in cart $^{1,1}\left(B^{n} \times S^{1}\right)$ have a unique representative, and the energies $\mathcal{E}_{1,1}(T)$ and $\mathcal{F}_{1,1}(T)$ are equal, i.e., no gap phenomenon occurs. Moreover, if $x$ belongs to the jump-concentration set $J_{c}(T)$, the 1-dimensional restriction has the form

$$
\widehat{\pi}_{\#}\left(T\left\llcorner\{x\} \times S^{1}\right)=\llbracket \gamma_{x} \rrbracket+q \llbracket S^{1} \rrbracket,\right.
$$

where $q \in \mathbb{Z}$ and $\llbracket \gamma_{x} \rrbracket$ is the current associated to a suitably oriented simple arc $\gamma_{x}$ in $S^{1}$ connecting the points $u_{T}^{-}(x)$ and $u_{T}^{+}(x)$, where $u_{T}$ is the function in $B V\left(B^{n}, S^{1}\right)$ associated to $T$, and $\gamma_{x}=0$ if $x \notin J_{u_{T}}$. Consequently, in (7.5) we have

$$
\mathcal{L}_{T}(x)=\mathcal{H}^{1}\left(\gamma_{x}\right)+2 \pi|q|
$$

and hence in cart $^{1,1}\left(B^{n} \times S^{1}\right)$ the $B V$-energy agrees with the energy obtained in [13], compare Theorem 1 of [14, Vol. II, Section 6.2.3].

The singular set. If $u \in W^{1,1}\left(B^{n}, S^{1}\right)$, its singular set is the current $\mathbb{P}(u) \in$ $\mathcal{D}_{n-2}\left(B^{n}\right)$ given by

$$
\mathbb{P}(u)(\phi):=-\frac{1}{2 \pi} \partial G_{u}\left(\pi^{\#} \omega_{S^{1}} \wedge \pi^{\#} \phi\right)=\frac{1}{2 \pi} \int_{B^{n}} u^{\#} \omega_{S^{1}} \wedge d \phi
$$

for every $\phi \in \mathcal{D}^{n-2}\left(B^{n}\right)$, where

$$
\omega_{S^{1}}:=y^{1} d y^{2}-y^{2} d y^{1}
$$


is the volume 1-form in $S^{1} \subset \mathbb{R}^{2}$. Therefore, $\mathbb{P}(u)$ is the boundary of the current $\mathbb{D}(u) \in \mathcal{D}_{n-1}\left(B^{n}\right)$ defined for any $\gamma \in \mathcal{D}^{n-1}\left(B^{n}\right)$ by

$$
\mathbb{D}(u)(\gamma):=\frac{1}{2 \pi} G_{u}\left(\pi^{\#} \omega_{S^{1}} \wedge \pi^{\#} \gamma\right)=\frac{1}{2 \pi} \int_{B^{n}} u^{\#} \omega_{S^{1}} \wedge \gamma .
$$

Proposition 7.6. For every $u \in W^{1,1}\left(B^{n}, S^{1}\right)$ we have

$$
\mathbf{M}(\mathbb{D}(u)) \leq \frac{1}{2 \pi} \int_{B^{n}}|D u| d x .
$$

Proof. By the definition of mass we clearly infer

$$
2 \pi \mathbf{M}(\mathbb{D}(u)) \leq \int_{B^{n}}\left\|u^{\#} \omega_{S^{1}}\right\| d x .
$$

Moreover, since $u^{\#} \omega_{S^{1}}=u^{1} d u^{2}-u^{2} d u^{1}$, we estimate

$$
\left\|u^{\#} \omega_{S^{1}}\right\|^{2} \leq \sum_{i=1}^{n}\left|u^{1} u_{x_{i}}^{2}-u^{2} u_{x_{i}}^{1}\right|^{2} \leq \sum_{i=1}^{n}\left(\left|u^{1}\right|\left|u_{x_{i}}^{2}\right|+\left|u^{1}\right|\left|u_{x_{i}}^{2}\right|\right)^{2} .
$$

Observe now that for any $a, b>0$ and $\lambda, \mu>0$ with $\lambda^{2}+\mu^{2}=1$

$$
\lambda a+\mu b \leq \sqrt{a^{2}+b^{2}} .
$$

Since $|u(x)|=1$, this yields $\left(\left|u^{1}\right|\left|u_{x_{i}}^{2}\right|+\left|u^{1}\right|\left|u_{x_{i}}^{2}\right|\right)^{2} \leq\left|D_{x_{i}} u\right|^{2}$ and hence the assertion.

We now recover the following estimates about the relaxed energy, compare [8] and [19].

Proposition 7.7. For every $u \in W^{1,1}\left(B^{n}, S^{1}\right)$ we have

$$
\widetilde{\mathcal{E}_{1,1}}(u) \leq 2 \mathcal{E}_{1,1}(u), \quad \text { where } \quad \mathcal{E}_{1,1}(u):=\int_{B^{n}}|D u| d x .
$$

Moreover, for every $u \in B V\left(B^{n}, S^{1}\right)$ we have

$$
\widetilde{\mathcal{E}_{T V}}(u) \leq 2 \mathcal{E}_{T V}(u),
$$

where $\mathcal{E}_{T V}(u)$ is the total variation of $u$, given by (6.1).

Proof. Let $u \in W^{1,1}\left(B^{n}, S^{1}\right)$. Proposition 7.6 yields that the real mass $m_{r, B^{n}}(\mathbb{P}(u)) \leq$ $\mathcal{E}_{1,1}\left(u, B^{n}\right) / 2 \pi$ and hence, on account of Hardt-Pitts' result (7.1), the integral mass

$$
m_{i, B^{n}}(\mathbb{P}(u)) \leq \frac{1}{2 \pi} \mathcal{E}_{1,1}(u),
$$


see Definition 7.2. As a consequence, since for every $\varepsilon>0$ we find a current $T \in \mathcal{T}_{u}$ such that

$$
T=G_{u}+L \times \llbracket S^{1} \rrbracket \quad \text { and } \quad \mathcal{E}_{1,1}(T)=\mathcal{E}_{1,1}(u)+2 \pi \mathbf{M}(L),
$$

where $L \in \mathcal{R}_{n-1}\left(B^{n}\right)$ satisfies $\mathbf{M}(L) \leq m_{i, B^{n}}(\mathbb{P}(u))+\varepsilon$, taking into account Theorem 7.4 we obtain (7.8).

In the more general case $u \in B V\left(B^{n}, S^{1}\right)$, Theorem 6.7 yields the existence of a sequence $\left\{u_{k}\right\} \subset W^{1,1}\left(B^{n}, S^{1}\right)$ such that $u_{k} \rightarrow u$ weakly in the $B V$-sense and $\mathcal{E}_{1,1}\left(u_{k}\right) \rightarrow \mathcal{E}_{T V}(u)$. Also, for every $k$ we find a smooth sequence $\left\{u_{h}^{(k)}\right\}_{h} \subset$ $C^{1}\left(B^{n}, S^{1}\right)$ converging to $u_{k}$ strongly in $L^{1}$ and such that $\mathcal{E}_{1,1}\left(u_{h}^{(k)}\right) \rightarrow \widetilde{\mathcal{E}_{1,1}}\left(u_{k}\right)$ $+1 / k$ as $h \rightarrow \infty$. Finally, by (7.8) and by a diagonal argument we readily obtain (7.9).

Remark 7.8. As in [20], since $\pi_{1}(\mathcal{Y})$ is commutative, if $u \in R_{1}^{\infty}\left(B^{n}, \mathcal{Y}\right)$, for every $s=1, \ldots, \bar{s}$ we may find an integral current $L_{s} \in \mathcal{R}_{n-2}\left(B^{n}\right)$ satisfying

$$
(-1)^{n}\left(\partial L_{s}\right)\left\llcorner B^{n}=\mathbb{P}_{S}(u) \quad \text { and } \quad \mathbf{M}\left(L_{s}\right) \leq C \int_{B^{n}}|D u| d x\right.
$$

for some absolute constant $C>0$ independent of $u$. Therefore, arguing as above it is not difficult to show that

$$
\widetilde{\mathcal{E}_{1,1}}(u) \leq C(n, \mathcal{Y}) \cdot \mathcal{E}_{1,1}(u) \quad \forall u \in W^{1,1}\left(B^{n}, \mathcal{Y}\right)
$$

where $C(n, \mathcal{Y})>0$ is an absolute constant, only depending on $n$ and $\mathcal{Y}$. Finally, by Theorem 6.7 we conclude that

$$
\widetilde{\mathcal{E}_{T V}}(u) \leq C(n, \mathcal{Y}) \cdot \mathcal{E}_{T V}(u) \quad \forall u \in B V\left(B^{n}, \mathcal{Y}\right),
$$

where $\mathcal{E}_{T V}(u)$ is the total variation given by (6.1) and the optimal constant $C(n, \mathcal{Y})$ is the same as the optimal constant for $W^{1,1}$-functions in (7.10).

\section{References}

[1] L. Ambrosio, Metric space valued functions of bounded variation, Ann. Scuola Norm. Sup. Pisa Cl. Sci. 17 (1990), 439-478.

[2] L. Ambrosio, N. Fusco and D. Pallara, "Functions of bounded Variation and Free Discontinuity Problems", Oxford Math. Monographs, Oxford, 2000.

[3] L. Ambrosio and B. Kirchheim, Currents in metric spaces, Acta Math. 185 (2000), $1-80$.

[4] P. Aviles and Y. GigA, Variational integrals of mappings of bounded variation and their lower semicontinuity, Arch. Ration. Mech. Anal. 115 (1991), 201-255.

[5] F. BethuEL, The approximation problem for Sobolev maps between manifolds, Acta Math. 167 (1992), 153-206.

[6] F. Bethuel, J. M. Coron, F. Demengel and F. Helein, A cohomological criterium for density of smooth maps in Sobolev spaces between two manifolds, In: "Nematics, Mathematical and Physical Aspects", J. M. Coron, J. M. Ghidaglia, F. Helein (eds.), NATO ASI Series C, 332, Kluwer Academic Publishers, Dordrecht, 1991, 15-23. 
[7] G. Bouchitté and G. ButTAZzo, New lower semicontinuity results for nonconvex functionals defined on measures, Nonlinear Anal. 15 (1990), 679-692.

[8] H. Brezis, P. Mironescu and A. Ponce, $W^{1,1}$-maps with value into $S^{1}$, In: "Geometric Analysis of PDE and Several Complex Variables", S. Chanillo, P. Cordaro, N. Hanges and A. Meziani (eds.), Contemporary Mathematics, 368, American Mathematical Society, Providence, RI, 2005, 69-100.

[9] H. FederER, "Geometric Measure Theory", Grundlehren math. Wissen. 153, Springer, Berlin, 1969.

[10] H. FEDERER, Real flat chains, cochains and variational problems, Indiana Univ. Math. J. 24 (1974), 351-407.

[11] E. Gagliardo, Caratterizzazione delle tracce sulla frontiera relative ad alcune classi di funzioni in $n$ variabili, Rend. Sem. Mat. Univ. Padova 27 (1957), 284-305.

[12] M. Giaquinta and G. ModicA, On sequences of maps with equibounded energies, Calc. Var. Partial Differential Equations 12 (2001), 213-222.

[13] M. Giaquinta, G. ModiCA and J. SOUČEK, Variational problems for maps of bounded variations with values in $S^{1}$, Calc. Var. 1 (1993), 87-121.

[14] M. Giaquinta, G. ModiCA and J. SouČEK, "Cartesian Currents in the Calculus of Variations", I, II. Ergebnisse Math. Grenzgebiete (III Ser), 37, 38, Springer, Berlin, 1998.

[15] M. GIAQUinTA and D. MuCCI, Weak and strong density results for the Dirichlet energy, J. Eur. Math. Soc. 6 (2004), 95-117.

[16] M. GIAQUINTA and D. MUCCI, The Dirichlet energy of mappings from $B^{3}$ into a manifold: density results and gap phenomenon, Calc. Var. Partial Differential Equations 20 (2004), 367-397.

[17] M. Giaquinta and D. MuCCI, On sequences of maps into a manifold with equibounded $W^{1 / 2}$-energies, J. Funct. Anal. 225 (2005), 94-146.

[18] B. HARDT and J. PITTS, Solving the Plateau's problem for hypersurfaces without the compactness theorem for integral currents, In: "Geometric Measure Theory and the Calculus of Variations", W. K. Allard and F. J. Almgren (eds.), Proc. Symp. Pure Math. 44. Amer. Math. Soc., Providence, 1996, 255-295.

[19] R. IGNAT, The space $B V\left(S^{2} ; S^{1}\right)$ : minimal connections and optimal liftings Ann. Inst. $\mathrm{H}$. Poincaré Anal. Non Linéaire 22 (2005), 283-302.

[20] M. R. PAKZAD and T. RIVIÈRE, Weak density of smooth maps for the Dirichlet energy between manifolds, Geom. Funct. Anal. 13 (2001), 223-257.

[21] R. SCHOEN and K UHLENBECK, Boundary regularity and the Dirichlet problem for harmonic maps, J. Differential Geom. 18 (1983), 253-268.

[22] L. Simon, "Lectures on geometric measure theory", Proc. C.M.A., Vol. 3, Australian Natl. Univ., Canberra, 1983.

[23] B. White, Rectifiability of flat chains, Ann. of Math. (2) 150 (1999), 165-184.

Scuola Normale Superiore, Piazza dei Cavalieri 7 I-56100 Pisa, Italy giaquinta@sns.it

Dipartimento di Matematica dell'Università di Parma Viale G. P. Usberti 53/A I-43100 Parma, Italy domenico.mucci@unipr.it 